TE WHARE WĀNANGA O TE ŪPOKO O TE IKA A MĀUI

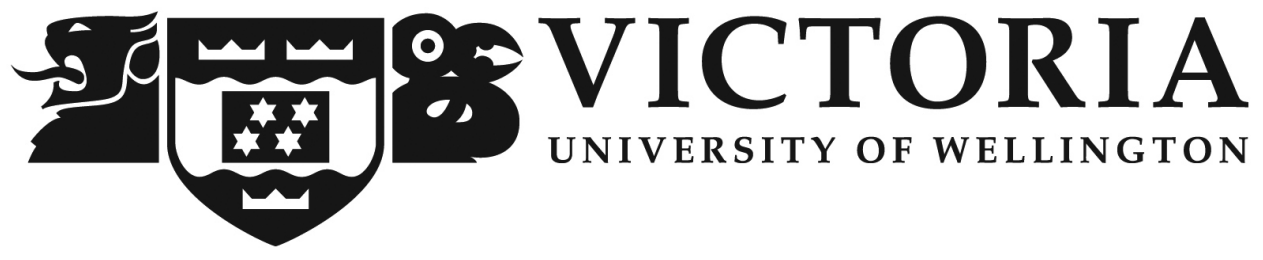

\title{
Magneto-tunnelling transport of chiral charge carriers
}

MSc Thesis

\author{
Luke Pratley \\ BSc (Hons)
}

Supervisor:

Prof. Ulrich Zülicke

A thesis submitted to the Victoria University of Wellington in

fulfillment of the requirements for the degree of Master of Science in

Physics. Victoria University of Wellington 2014 


\section{Acknowledgements}

I thank my supervisor Prof. Ulrich Zülicke, for aiding and guiding me in my research. I thank the theoretical condensed matter physics research group at Victoria University of Wellington for the weekly group meetings, particularly the members who engaged in my presentations. I would like to thank the friendly students and staff who I have met while performing research. I would like to thank Dr. Melanie Johnston-Hollitt for her comments on my thesis. Lastly, I would like to thank my family for being supportive.

\section{Refereed publications based on work in this thesis:}

- [1] L. Pratley and U. Zülicke, Magnetotunneling spectroscopy of chiral twodimensional electron systems, Phys. Rev. B 88, 245412 (2013)

- 2] L. Pratley and U. Zülicke, Valley filter from magneto-tunneling between single and bi-layer graphene, Applied Physics Letters 104, 082401 (2014) 


\begin{abstract}
We study magneto-tunnelling between two parallel two-dimensional electron gases theoretically, where the electrons have a pseudo-spin- $\frac{1}{2}$ degree of freedom that is coupled to their momentum. The two-dimensional electron gases focused on in this work are single layer graphene, bilayer graphene, and single layer molybdenum disulphide.

The results are derived using a linear response theory formalism in the weak tunnelling regime, and it is assumed that the electron gases are at zero temperature, with no interactions or disorder. The linear magneto-tunnelling conductance characteristics for an applied in-plane and tilted magnetic field are found to strongly depend on the pseudo-spin structure of the tunnelling matrix and the pseudo-spin's dependence on momentum. For instance, resonances in the linear magneto-tunnelling conductance are sensitive to the pseudo-spin tunnel-coupling across the barrier and how the pseudo-spin eigenstates are coupled to momentum.

We discuss how measurements of the magneto-tunnelling conductance can be applied as a spectroscopic tool. We explain how to measure the pseudo-spin tunnelcoupling through least squares parameter fitting of the magneto-tunnelling conductance. We show that the parameters are interdependent, one can use the interdependency to test the consistency between theory and experiment. It is expected that measurements of pseudo-spin tunnel-coupling will be a function of the lattice structure of the double layer system, which suggests these measurements can be used as a spectroscopic tool. Additionally, we investigate in-plane electric fields in single layer graphene to see if their effects can be observed in magneto-tunnelling transport. Then, we perturbatively include the effects of electron-electron interactions in single layer graphene, and find it should dampen the linear tunnelling conductance.

We investigate tunnel-coupled, parallel, single layer and bilayer graphene systems. We find that using an in-plane magnetic field, one can generate a valley polarized tunnelling current. This method is unique because it does not require manipulation of the single and bilayer graphene samples through nano-structuring, coupling to electromagnetic fields, application of mechanical strain, or the presence of defects. In particular, the valley polarization is dependent on the pseudo-spin tunnel-coupling between the single and bilayer graphene systems, and the strength of an applied in-plane magnetic field.

We explicitly show through analytic derivations how an understanding of linear magneto-tunnelling transport (zero bias limit) can be used to understand non-linear magneto-tunnelling transport (finite bias).
\end{abstract}




\section{Contents}

\begin{tabular}{lll}
\hline & Introduction & 1
\end{tabular}

$\begin{array}{|lll|}2 & \text { Band structure of two-dimensional chiral conductors } & 7\end{array}$

2.1 Single layer graphene . . . . . . . . . . . . . . . . . . . . . . . . . . . . . . . . 8

2.2 Bilayer graphene . . . . . . . . . . . . . . . . . . . . . . . . . 9

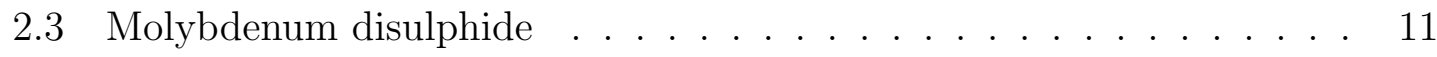

2.4 Carrier concentration and density of states at the Fermi energy. . . . 12

$\begin{array}{lll}3 & \text { Theory of magneto-tunnelling transport } & 14\end{array}$

$3.1 \quad$ Linear response theory for tunnelling transport . . . . . . . . . . . . 14

3.2 Tunnelling between two-dimensional non-chiral electron gases. . . . . . 17

3.2.1 The effect of an in-plane magnetic field . . . . . . . . . . . . . 18

3.2 .2 The effect of a tilted magnetic field . . . . . . . . . . . . . . . 21

3.2.3 The effect of an in-plane magnetic field with a finite bias . . . 22

4 Magneto-tunnelling between two dimensional chiral electron gases 26

4.1 Effect of an in-plane magnetic field . . . . . . . . . . . . . . . . 26

$4.1 .1 \quad$ Form of the tunnelling matrix . . . . . . . . . . . . . . . 26

4.1.2 Linear magneto-tunnelling conductance formula . . . . . . . . 27

4.2 Pseudo-spin tunnelling matrix elements . . . . . . . . . . . . . . . . . 30

$4.2 .1 \quad$ Single layer graphene . . . . . . . . . . . . . . . . . . . . . . . . . . . . . . . . . . . . . 30

4.2 .2 Bilayer graphene . . . . . . . . . . . . . . . . . . 33

4.2 .3 Tunnelling matrix parametrisation . . . . . . . . . . . . . . 34

4.3 Comparative study of chiral magneto-tunnelling transport . . . . . . 36

$4.3 .1 \quad$ Single layer graphene . . . . . . . . . . . . . . . . . . . . . . . . . . . 36

4.3 .2 Bilayer graphene . . . . . . . . . . . . . . . . . . . . . . . . . . . . . . . . 37

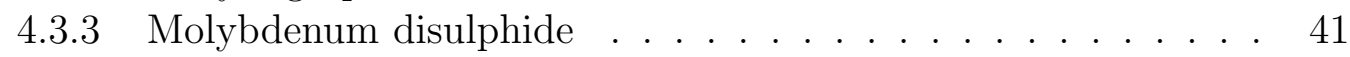

4.3 .4 Experimental requirements. . . . . . . . . . . . . . . . . . . . . . . . . 43

4.4 Landau levels in single layer graphene . . . . . . . . . . . . . . . . . . 43

$4.4 .1 \quad$ Linear magneto-tunnelling conductance in tilted magnetic field 44

$4.4 .2 \quad$ Effect of a perpendicular magnetic field . . . . . . . . . . . . . 46

5 Magneto-tunnelling as a spectroscopic tool $\quad 49$

$5.1 \quad$ Experimental measurement of tunnel-coupling . . . . . . . . . . . . . 49

$5.1 .1 \quad$ Relating $\tau$ to sub-lattice tunnel-coupling . . . . . . . . . . . . 51

$5.1 .2 \quad$ Alignment of sub-lattices and structure of the barrier . . . . . 52

$5.2 \quad$ Valley polarisation of current between single and bilayer graphene . . 53

5.2.1 Effective tunnel coupling between single layer and bilayer graphene 54

5.2 .2 Generation of a valley polarisation . . . . . . . . . . . 56 


\section{CONTENTS}

$5.3 \quad$ Effect of an in-plane electric field on magneto-tunneling current . . . 59

$5.3 .1 \quad$ Electric field perpendicular to the in-plane magnetic field . . . 59

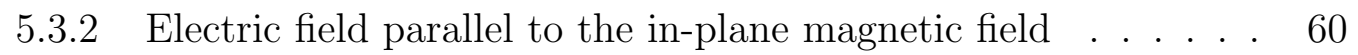

$5.3 .3 \quad$ Noticeable effects of an in-plane electric field . . . . . . . . . . . 62

5.4 Measuring interaction renormalisation of Fermi velocity . . . . . . . 63

6 Non-linear magneto-tunnelling transport $\quad 66$

\begin{tabular}{lll}
\hline 7 & Conclusion & 71
\end{tabular} 


\section{Chapter 1}

\section{Introduction}

In 2004, Andre Geim and Konstantin Novoselov separated the first one-atom-thick layer of graphite, called graphene, and explored the unusual material's electronic properties [3]. Graphene was found to be the thinnest and strongest material known to date, as well as being an excellent transparent conductor [4]. These novel properties have provided hope for graphene to be used in a variety of applications. However, there is also a purely scientific interest in graphene. In particular, graphene's honeycomb lattice of carbon atoms provides a linear dispersion relation, simulating the effects of quantum physics and Einstein's theory of relativity in a two-dimensional electronic device [5, 6]. Just as a massless particle in three spatial dimensions follows the three dimensional Dirac equation, an electron in graphene also follows a Dirac like equation in two dimensions.

The band structure of graphene has two valleys in the Brillouin zone where low energy excitations are possible. These valleys are known as the $\mathbf{K}$ and $\mathbf{K}^{\prime}$ valleys. Each valley is characterised by a Dirac-cone-like energy-momentum relation $\varepsilon=$ $\pm v\|\mathbf{p}\|$, where $v$ is the Fermi velocity of graphene. The valleys are centred at the points where the conduction and valence bands meet, as shown in Fig. 1.1.

One can show that a honeycomb lattice consists of two equivalent sub-lattices, which are labelled $\mathrm{A}$ and $\mathrm{B}$, as in Fig. 1.2. In particular, an electron in graphene can occupy the $p_{z}$-orbitals of either sub-lattice A or sub-lattice B. One can employ a pseudo-spin- $\frac{1}{2}$ representation where $|A\rangle=\left(\begin{array}{l}1 \\ 0\end{array}\right)$ and $|B\rangle=\left(\begin{array}{l}0 \\ 1\end{array}\right)$. These two quantum states span a two-dimensional Hilbert space, analogous to the intrinsic spin of an electron. For this reason, we call the states associated with occupied sub-lattices pseudo-spin.

The pseudo-spin eigenstates for electrons in graphene are coupled to the direction of the momentum eigenstates via the Hamiltonians [7]

$$
\mathcal{H}_{\mathbf{K}}=v \sigma_{x} p_{x}+v \sigma_{y} p_{y}, \quad \mathcal{H}_{\mathbf{K}^{\prime}}=-v \sigma_{x} p_{x}+v \sigma_{y} p_{y}
$$

where $\sigma_{x}, \sigma_{y}, \sigma_{z}$ are the Pauli matrices for pseudo-spin. More details on graphene can be found in Ref. [8].

In recent years, there has been progress in the fabrication of other two-dimensional crystals, such as bilayer graphene, hexagonal boron nitride (h-BN), molybdenum disulphide $\left(\mathrm{MoS}_{2}\right)$, and tungsten disulphide $\left(\mathrm{WS}_{2}\right)$ [11. Each two-dimensional crystal has different electronic properties. For example, h-BN is an insulator and $\mathrm{MoS}_{2}$ is a semi-conductor. Further more, it is possible to layer these materials on 


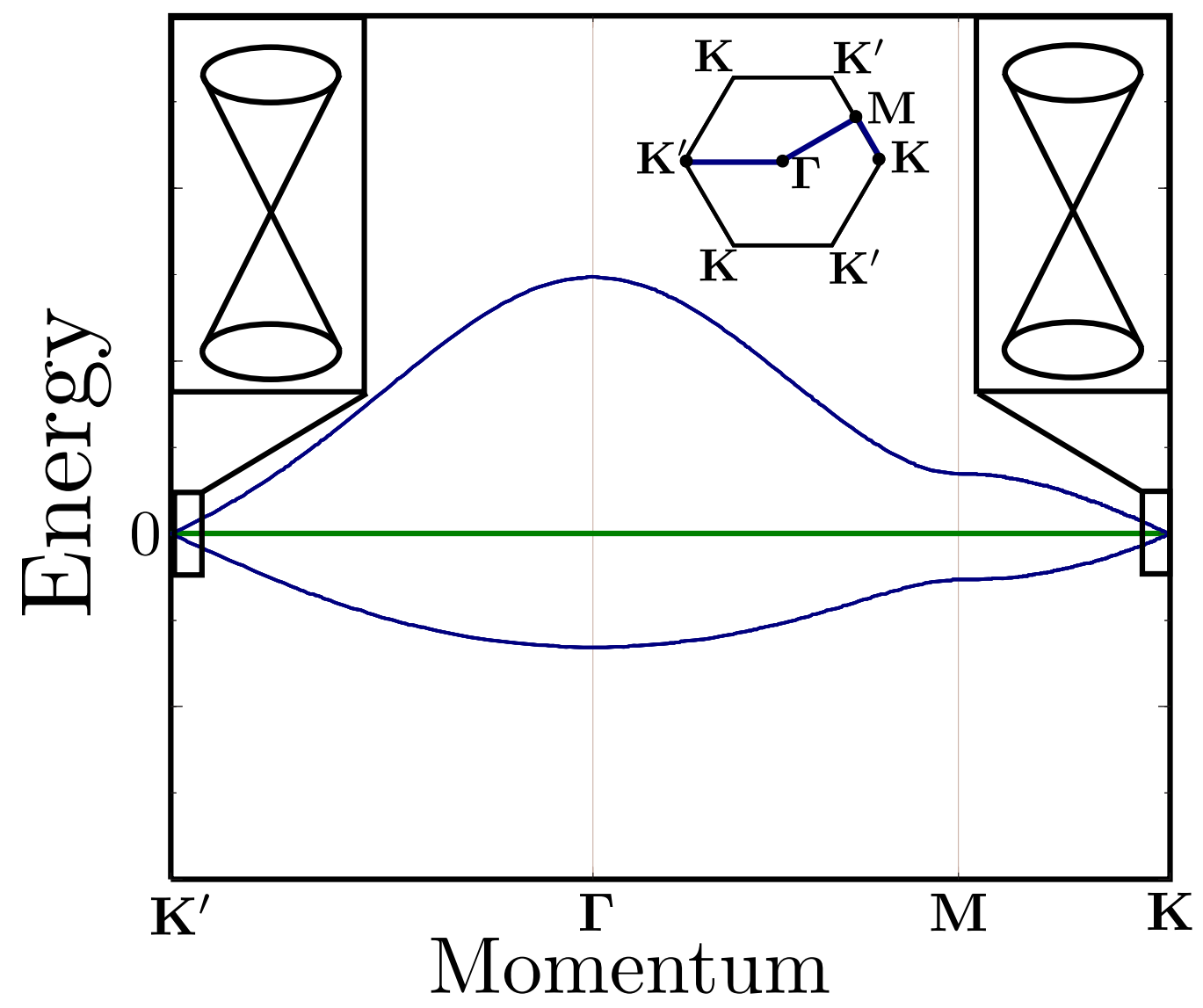

Figure 1.1: The band structure of graphene. The hexagon represents the Brillouin zone for graphene, and the blue line indicates the direction in which momentum varies in the energy-momentum plot. We can see the $\mathbf{K}$ and $\mathbf{K}^{\prime}$ valleys at each corner of the Brillouin zone. Near these valleys, the energy-momentum relation becomes linear, a shown by the Dirac cones. The green line corresponds to the Fermi energy.

a)

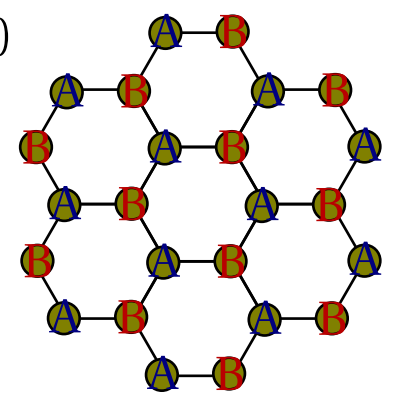

b)

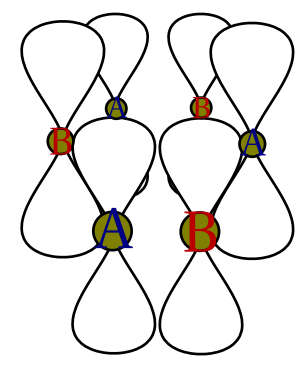

Figure 1.2: a) Diagram of the carbon honeycomb lattice of graphene, where each member of a sub-lattice has been labelled A or B. An electron can occupy one of the sub-lattices of $p_{z}$-orbitals, shown in b). 
top of each other, in what is called a van der Waals heterostructure[12]. Materials used in van der Waals heterostructures have lattice structures similar to graphene. Each material has a pseudo-spin structure, and each material can be reduced to a single atomic layer.

Quantum particles such as electrons often carry discrete degrees of freedom such as their real (intrinsic) spin or pseudo-spin, which have properties of angular momentum. In certain situations, the dynamics of the (pseudo-)spin is rigidly coupled to the particle's orbital motion. We call systems in which such a spin-momentum locking exists chiral. The charge carriers in graphene form a chiral system in their sublattice-related pseudo-spin degree of freedom. See Fig. 1.4 where it is illustrated how the pseudo-spin direction (more precisely; its quantisation axis) depends on the direction of the electron momentum. Other examples of chirality can be found from the Dirac equation [13] and from Rashba spin-orbit coupling [14].

The examples of chiral materials we will focus on can be used in van der Waals heterostructures, these are single layer graphene, bilayer graphene, and $\mathrm{MoS}_{2}$.

A popular van der Waals heterostructure is two layers of graphene separated by an atomically thin insulating barrier (approx. $3 \mathrm{~nm}$ [15]), such as h-BN (stacked in the order graphene/h-BN/graphene) [15-19]. This double layer van der Waals heterostructure has been used in coulomb drag experiments [20], and as the core component of the new device known as a vertical field effect transistor [15 18]. But other than Ref. [21], little work has been done to learn about the tunnelling of chiral charge carriers across a double layer van der Waals heterostructure in the presence of both electric and magnetic fields. We are filling this void by extending previous work [22 25] on magneto-tunnelling spectroscopy. See Ref. [1, 2] for a record of the published results in this thesis.

It is well known that tunnelling spectroscopy is an effective tool for investigating electronic structure [26, 27]. Tunnelling spectroscopy is well known for being used to measure the density of states. Typically a well understood contact system, such as a tip, is put in tunnelling contact with a sample material. In order to measure the density of states in the sample material, one needs to assume zero temperature, a constant tunnelling matrix element, and a constant density of states in the contact material. Under these assumptions, the tunnelling conductance between the contact material and the sample material is proportional to the sample's density of states.

When tunnelling occurs through a uniform extended barrier, the momentum component that is parallel to the barrier must be conserved in a tunnelling event. The condition of simultaneous energy and momentum conservation enables tunneling spectroscopy to be used to measure a material's band structure. Due to energy and canonical momentum conservation in the tunnelling process, tunnelling can only occur whenever the energy-momentum curves of the two systems intersect. One can apply a bias and magnetic field to separate the two energy-momentum curves. A description of this is shown in Fig. 1.3. When the two energy-momentum curves start/cease to intersect, the tunnelling current is switching on/off. This switching on or off is a sharp discontinuity in the tunnelling current, which creates a resonance (or divergence) in the magneto-tunnelling conductance. These resonances and 
other features of the magneto-tunnelling conductance can be used to probe the band structure of the two systems.

Momentum-resolved tunnelling has been used in the context of parallel twodimensional quantum wells [28 34], parallel quantum wires [35-38], and quantum dots [39], where it has shown success for measuring the energy-momentum relations [40 42]. However, none of these works include materials with pseudo-spin eigenstates that are coupled to momentum. This provided us with the opportunity to apply and extend methods used in previous works, to research that is relevant at present.

Since pseudo-spin states are determined by the lattice structure of the twodimensional systems, the atomic lattice structure of the barrier will alter possible magneto-tunnelling processes for a double layer van der Waals heterostructure. We show later that an applied magnetic field will alter the chiral pseudo-spin eigenstates in a van der Waals heterostructure. These features of double layer van der Waals heterostructures suggest that there is much to learn from studying their magnetotunnelling transport, motivating the work in this thesis.

We find that momentum-resolved tunnelling can be used to probe pseudo-spin structure of a graphene double layer van der Waals heterostructure. The magnetotunnelling conductance is sensitive to the pseudo-spin coupling between layers. This is particularly fascinating because the pseudo-spin states are determined by the sublattice structure of the two parallel systems. One can expect that the coupling will depend on the atomic structure of the barrier, and the alignment of sub-lattices between the two graphene systems.

In this work, we investigate the magneto-tunnelling conductance between two parallel two-dimensional chiral electron gases. We employ linear response theory, and extend methods used previously in the study of tunnelling between parallel two-dimensional non-chiral electron gases in the presence of an in-plane and tilted magnetic field. We look at how the magneto-tunnelling conductance may be used to investigate the influence of momentum dependent pseudo-spin in the tunnelling process, and allow one to probe the pseudo-spin structure of a double layer graphene van der Waals heterostructure. We also investigate effects of electron-electron interactions and in-plane electric fields on the magneto-tunnelling transport. We then look at the possibility of creating a valley-filter using single layer and bilayer graphene in the presence of an in-plane magnetic field. The possibility of a valley-filter would allows one to control the valley degree of freedom for electrons in graphene for information processing, allowing one to create valleytronic devices 43 45]. Lastly, we discuss the finite bias magneto-tunnelling current and conductance for single layer graphene.

The layout of this thesis is as follows. In chapter 2, we introduce the pseudospin and band structure for single layer graphene, bilayer graphene, and $\mathrm{MoS}_{2}$. In chapter 3, we introduce the formalism for calculating the tunnelling current and conductance. We also review previous work for magneto-tunnelling between two parallel non-chiral two-dimensional electron gases. This includes the effect of an inplane and titled magnetic field with a small bias between the layers, then the effect of an in-plane magnetic field with a finite bias between the layers. In chapter 4 , we 


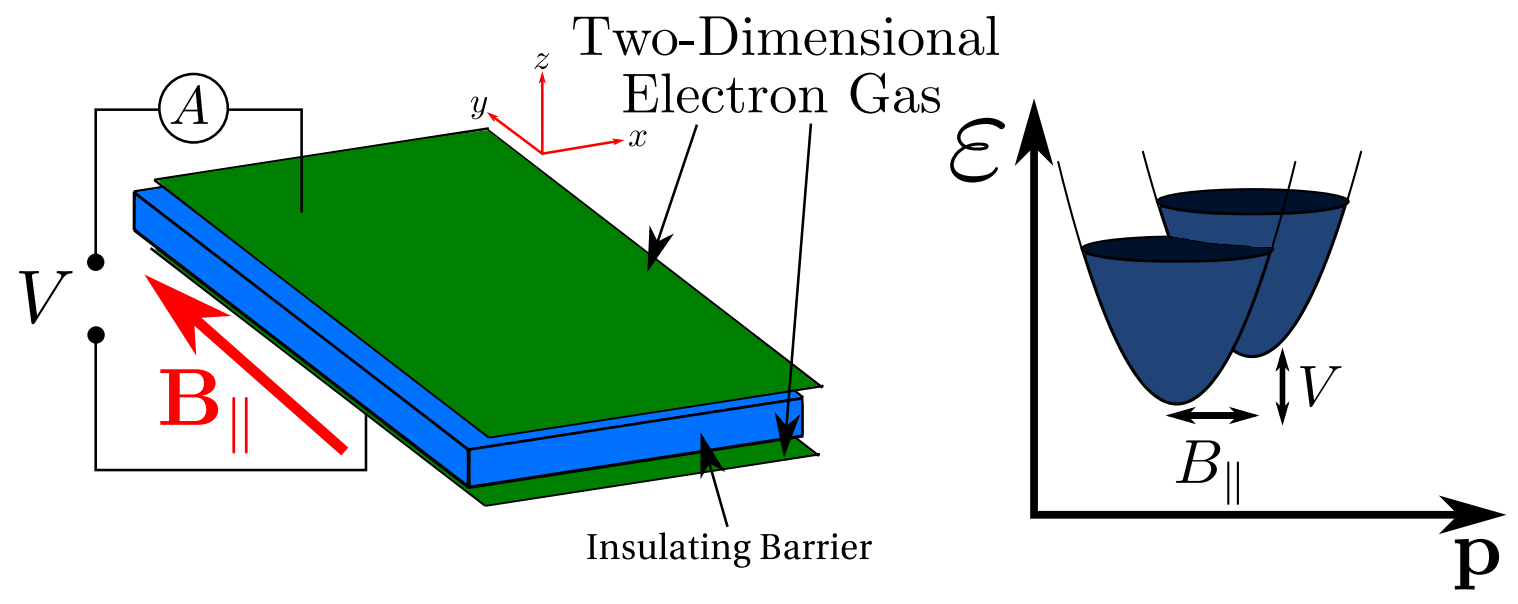

Figure 1.3: (left) Two parallel two-dimensional electron gases, separated by an insulating tunnelling barrier. A bias $V$ is applied between the layers, and an in-plane magnetic field $\mathbf{B}_{\|}$is applied. (right) Two intersecting energy momentum relations for the system on the left. Energy and canonical momentum conservation in the tunnelling process means that the magneto-tunnelling conductance between the two layers is determined by the intersection of the energy-momentum relations, which have been shifted by the applied bias (shifts energy) and magnetic field (shifts kinetic momentum).
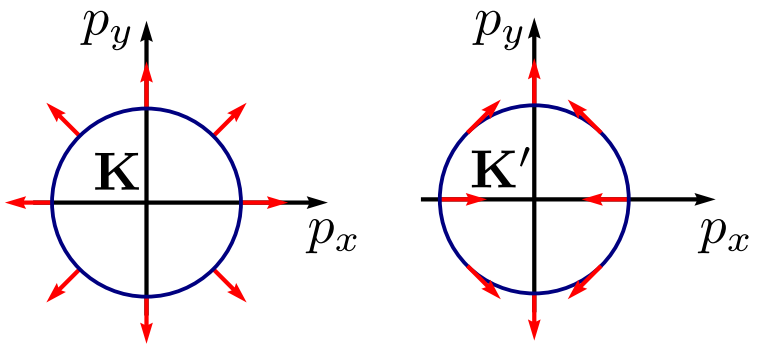

Figure 1.4: A figure showing how the direction of momentum determines the direction of pseudo-spin. The red arrows show the direction of the positive energy pseudo-spin eigenstates for electrons in graphene, for momentum states on the blue circle. For the $\mathbf{K}$ valley, the eigenstate is parallel to the direction of momentum $\left(p_{x}, p_{y}\right)$. In the $\mathbf{K}^{\prime}$ valley, the eigenstate is parallel to $\left(-p_{x}, p_{p}\right)$. 
investigate magneto-tunnelling between two parallel chiral two-dimensional electron gases. In particular, chapter 4 extends the work reviewed in chapter 3. In chapter 5, we discuss using magneto-tunnelling as a spectroscopic tool. We suggest how one could use the work in chapter 4 to experimentally probe the tunnel-coupling between two single layer graphene systems. We propose how single layer graphene and bilayer graphene can be used to create a valley filter. We also investigate the effects of an in-plane electric field and interactions for magneto-tunnelling in a double layer graphene system. In chapter 6, we look at the effects of an in-plane magnetic field on magneto-tunnelling between two parallel single graphene layers with a finite bias. This extends the finite bias work in chapter 3 for two-dimensional chiral electron gases. In chapter 7 we conclude this thesis, and suggest possible future works. 


\section{Chapter 2}

\section{Band structure of two-dimensional chiral conductors}

In this chapter we present the Hamiltonians on which later calculations will be based on, for electrons in single layer graphene (SLG), bilayer graphene (BLG), and $\mathrm{MoS}_{2}$ at the $\mathbf{K}$ and $\mathbf{K}^{\prime}$ valleys. These Hamiltonians are valid at low energies. Each of these two-dimensional chiral electron gases can be used in a van der Vaals double layer heterostructure, and are studied later in this work.

At the $\mathbf{K}$ and $\mathbf{K}^{\prime}$ valleys, the axis of the pseudo-spin eigenstates depends on the direction of momentum. The $\mathbf{K}$ and $\mathbf{K}^{\prime}$ valleys follow the same energy-momentum relation, but the pseudo-spin eigenstates at each valley have a different dependence on momentum.

Since two valleys can be occupied, the Hamiltonian for system $m$ at low energies will be of the form

$$
\mathcal{H}^{(m)}=\left(\begin{array}{cc}
\mathcal{H}_{\mathbf{K}}^{(m)} & 0 \\
0 & \mathcal{H}_{\mathbf{K}^{\prime}}^{(m)}
\end{array}\right) .
$$

$\mathcal{H}_{\mathbf{K}}$ and $\mathcal{H}_{\mathbf{K}^{\prime}}$ are $2 \times 2$ Hamiltonians at the $\mathbf{K}$ and $\mathbf{K}^{\prime}$ valley respectively. Each has single particle eigenstates that follow

$$
\mathcal{H}_{\gamma}^{(m)}\left|\psi_{\gamma, \mathbf{k}, \sigma}^{(m)}\right\rangle=\varepsilon_{\gamma, \mathbf{k}, \sigma}^{(m)}\left|\psi_{\gamma, \mathbf{k}, \sigma}^{(m)}\right\rangle,
$$

$\sigma= \pm$ represents the positive and negative (conduction and valence band) energy pseudo-spin eigenstate. $\gamma=\mathbf{K}, \mathbf{K}^{\prime}$ represents the valley for the eigenstate.

The pseudo-spin eigenstates are chiral, which means they are a function of the momentum eigenstates. For a particle in the presence of a magnetic field, one can write this as

$$
\left|\psi_{\gamma, \mathbf{k}, \sigma}(\mathbf{B})\right\rangle=|\mathbf{k}\rangle \otimes|\sigma\rangle_{\gamma, \boldsymbol{\Pi}(\mathbf{k} . \mathbf{B})} .
$$

We use $\mathbf{p}=\hbar \mathbf{k}$ to denote the canonical momentum, e to denote the charge of an electron, and

$$
\mathbf{\Pi}(\mathbf{k}, \mathbf{B})=\hbar \mathbf{k}+e \mathbf{A}
$$

is the kinetic momentum which is implicitly a function of the external magnetic field $\mathbf{B}$ due to the vector potential $\mathbf{A}$. 


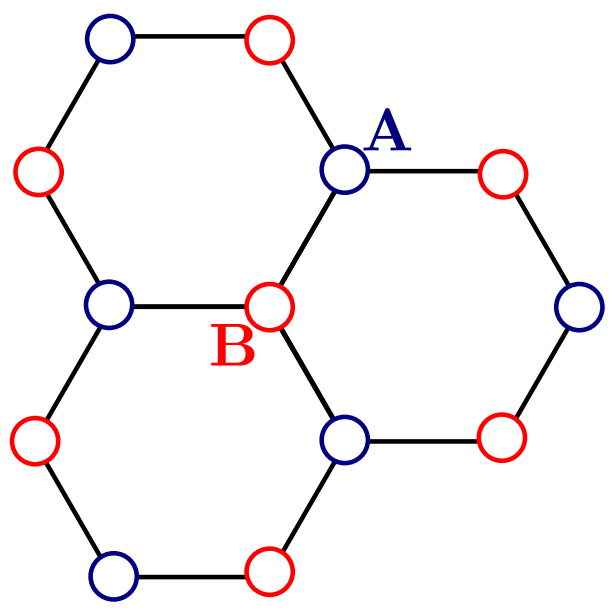

Figure 2.1: The honeycomb lattice structure of SLG. SLG can be split into two distinct sub-lattices $\mathbf{A}$ (blue) and $\mathbf{B}$ (red). These sub-lattices determine the pseudospin structure for SLG. This same structure is also shown in Fig. 1.2.

\subsection{Single layer graphene}

The honeycomb lattice structure of SLG can be seen in Fig. 2.1. This lattice can be split into two sub-lattices of equivalent points, sub-lattice $\mathbf{A}$ and $\mathbf{B}$. An electron occupying a given sub-lattice can be viewed as a pseudo-spin state, i.e. $\mathbf{A}=\left(\begin{array}{l}1 \\ 0\end{array}\right)$ and $\mathbf{B}=\left(\begin{array}{l}0 \\ 1\end{array}\right)$

The Hamiltonians at $\mathbf{K}$ and $\mathbf{K}^{\prime}$ valleys are

$$
\mathcal{H}_{\mathbf{K}}^{(\mathrm{slg})}=v \hbar k\left(\begin{array}{cc}
0 & \mathrm{e}^{-i \theta_{\mathbf{k}}} \\
\mathrm{e}^{i \theta_{\mathbf{k}}} & 0
\end{array}\right) \quad \text { and } \quad \mathcal{H}_{\mathbf{K}^{\prime}}^{(\mathrm{slg})}=v \hbar k\left(\begin{array}{cc}
0 & \mathrm{e}^{-i\left(\pi-\theta_{\mathbf{k}}\right)} \\
\mathrm{e}^{i\left(\pi-\theta_{\mathbf{k}}\right)} & 0
\end{array}\right)
$$

where $\theta_{\mathbf{k}}=\arctan \left(\frac{k_{y}}{k_{x}}\right)$ is the angle of momentum, $v$ is the Fermi velocity, and $k=\|\mathbf{k}\|$. It is clear that $\mathcal{H}_{\mathbf{K}}^{(\mathrm{slg})}$ is analogous to the two-dimensional Dirac equation for a massless particle, with an effective speed of $v$ [46]. As one would expect, for each valley we find a linear energy-momentum relation that is characteristic of a massless particle

$$
\varepsilon_{\gamma, \mathbf{k}, \sigma}^{(\mathrm{slg})}=\sigma v \hbar k
$$

The pseudo-spin eigenstates at each valley are

$$
|\sigma\rangle_{\mathbf{K}, \mathbf{k}}^{(\mathrm{slg})}=\frac{1}{\sqrt{2}}\left(\begin{array}{c}
\mathrm{e}^{-i \theta_{\mathbf{k}} / 2} \\
\sigma \mathrm{e}^{i \theta_{\mathbf{k}} / 2}
\end{array}\right) \quad|\sigma\rangle_{\mathbf{K}^{\prime}, \mathbf{k}}^{(\mathrm{slg})}=\frac{1}{\sqrt{2}}\left(\begin{array}{c}
\mathrm{e}^{-i\left(\pi-\theta_{\mathbf{k}}\right) / 2} \\
\sigma \mathrm{e}^{i\left(\pi-\theta_{\mathbf{k}}\right) / 2}
\end{array}\right) .
$$

Using these eigenstates, one can calculate the average pseudo-spin for each valley

$$
\mathbf{S}_{\gamma, \mathbf{k}, \sigma}^{(\mathrm{slg})}=\underset{\gamma, \mathbf{k}}{(\mathrm{slg})}\left\langle\sigma\left|\left(\sigma_{x}, \sigma_{y}, \sigma_{z}\right)\right| \sigma\right\rangle_{\gamma, \mathbf{k}}^{(\mathrm{slg})}
$$




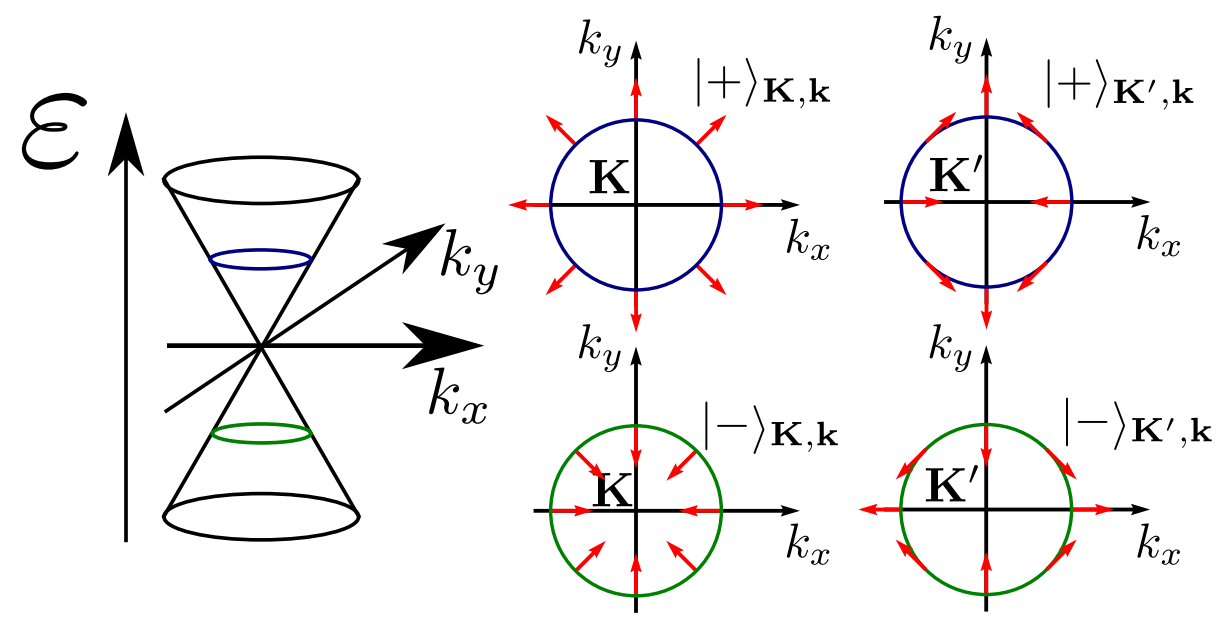

Figure 2.2: On the left is a Dirac cone centred at the $\mathbf{K}$ or $\mathbf{K}^{\prime}$ valley of SLG. On the right are slices of the cone for the conduction (blue) and valence (green) band for the $\mathbf{K}$ and $\mathbf{K}^{\prime}$ valleys. The red arrows show the direction of the pseudospin eigenstate $|\sigma\rangle_{\gamma, \mathbf{k}}$, relative to the direction of momentum. For the $\mathbf{K}$ valley, the positive (negative) pseudo-spin eigenstates point (anti-)parallel to the angle of momentum $\theta_{\mathbf{k}}$. The positive (negative) pseudo-spin eigenstate in the $\mathbf{K}^{\prime}$ valley points (anti-)parallel to the direction $\pi-\theta_{\mathbf{k}}$.

which determines the direction and axis of the pseudo-spin eigenstate. One finds the average pseudo-spin, for each eigenstate, at each valley as

$$
\mathbf{S}_{\mathbf{K}, \mathbf{k}, \sigma}^{(\mathrm{slg})}=\sigma\left(\cos \theta_{\mathbf{k}}, \sin \theta_{\mathbf{k}}, 0\right), \quad \mathbf{S}_{\mathbf{K}^{\prime}, \mathbf{k}, \sigma}^{(\mathrm{slg})}=\sigma\left(-\cos \theta_{\mathbf{k}}, \sin \theta_{\mathbf{k}}, 0\right) .
$$

The positive (negative) pseudo-spin eigenstate at the $\mathbf{K}$ valley is (anti-)parallel to the direction of momentum. The positive (negative) pseudo-spin eigenstates for the $\mathbf{K}^{\prime}$ valley point at the (anti-)parallel to the angle $\pi-\theta_{\mathbf{k}}$. One can see $\mathbf{S}_{\gamma, \mathbf{k}, \sigma}^{\text {(slg }}$ in Fig. 2.2 , where it is compared to the direction of momentum.

\subsection{Bilayer graphene}

BLG is made out of two stacked layers of SLG, as shown in Fig. 2.3. In fact, the Hamiltonian for BLG can be expressed in terms of two coupled SLG systems. One can write this Hamiltonian in terms of the sub-lattices $\left\{\mathbf{A}_{1}, \mathbf{B}_{1}, \mathbf{A}_{2}, \mathbf{B}_{2}\right\}$

$$
\mathcal{H}_{\mathbf{K}}^{(\mathrm{blg})}=\left(\begin{array}{cccc}
0 & v \hbar k_{+} & \gamma_{1} & 0 \\
v \hbar k_{-} & 0 & 0 & 0 \\
\gamma_{1} & 0 & 0 & v \hbar k_{-} \\
0 & 0 & v \hbar k_{+} & 0
\end{array}\right) .
$$

where $k_{ \pm}=k_{x} \pm i k_{y}$ [47]. We have labelled $\mathbf{A}_{1}$ and $\mathbf{A}_{2}$ as the two sub-lattices which overlap between the two SLG layers in BLG. This results in the coupling energy $\gamma_{1}$ between $\mathbf{A}_{1} \& \mathbf{A}_{2}$. To obtain a $2 \times 2$ BLG Hamiltonian, one assumes that $\mathbf{A}_{1}$ and 

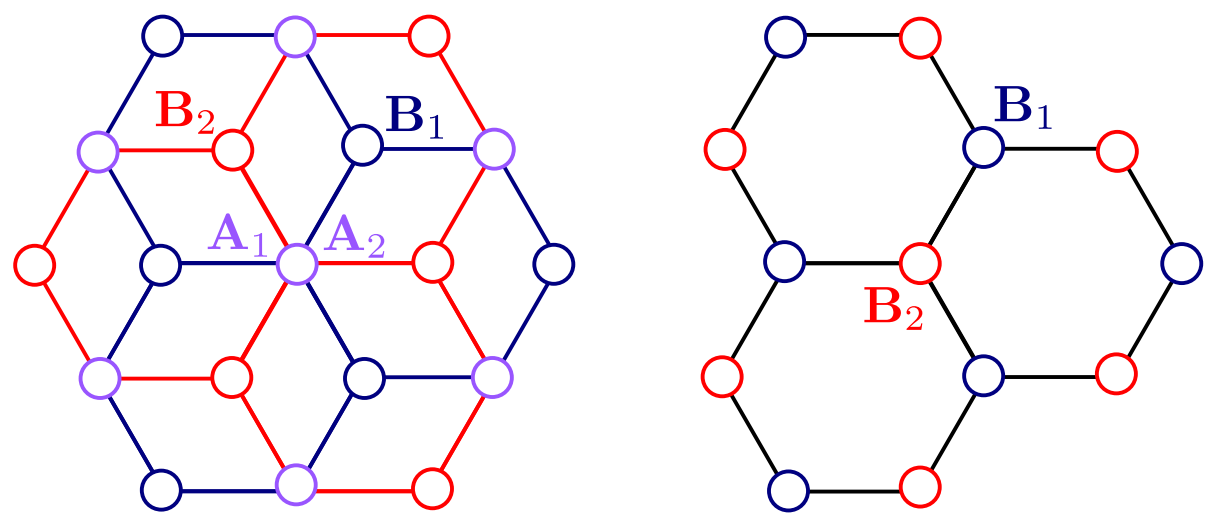

Figure 2.3: (left) The lattice structure of BLG, two stacked layers of SLG. Layer one (blue) is stacked on top of layer two (red). In purple, one can see sub-lattice $\mathbf{A}_{1}$ imposed on top of sub-lattice $\mathbf{A}_{2}$. (right) The effective BLG lattice at low energies. At low energies, the states $\mathbf{A}_{1}$ and $\mathbf{A}_{2}$ are not accessible. This reduces the system to the $\mathbf{B}_{1}$ and $\mathbf{B}_{2}$ sub-lattices.

$\mathbf{A}_{2}$ are inaccessible states, so that $\gamma_{1}$ is the largest energy scale of the system. One can then use Löwdin partitioning to project on the accessible states $\mathbf{B}_{1}$ and $\mathbf{B}_{2}$, to obtain the approximation

$$
\mathcal{H}_{\mathbf{K}}^{(\mathrm{blg})} \approx-\frac{(v \hbar)^{2}}{\gamma_{1}}\left(\begin{array}{cc}
0 & k_{+}^{2} \\
k_{-}^{2} & 0
\end{array}\right) .
$$

Each valley of BLG has a Hamiltonian of a massive chiral two-dimensional electron gas [48],

$$
\mathcal{H}_{\mathbf{K}}^{(\mathrm{blg})}=-\frac{k^{2}}{2 M}\left(\begin{array}{cc}
0 & \mathrm{e}^{i 2 \theta_{\mathbf{k}}} \\
\mathrm{e}^{-i 2 \theta_{\mathbf{k}}} & 0
\end{array}\right) \quad \text { and } \quad \mathcal{H}_{\mathbf{K}^{\prime}}^{(\mathrm{blg})}=-\frac{k^{2}}{2 M}\left(\begin{array}{cc}
0 & \mathrm{e}^{-i 2 \theta_{\mathbf{k}}} \\
\mathrm{e}^{i 2 \theta_{\mathbf{k}}} & 0
\end{array}\right) .
$$

The energy-momentum relation is parabolic, with an effective mass $M$

$$
\varepsilon_{\gamma, \mathbf{k}, \sigma}^{(\mathrm{blg})}=\sigma \frac{\hbar^{2}}{2 M} k^{2} .
$$

The pseudo-spin eigenstates for each valley are

$$
|\sigma\rangle_{\mathbf{K}, \mathbf{k}}=\frac{1}{\sqrt{2}}\left(\begin{array}{c}
\mathrm{e}^{i \theta_{\mathbf{k}}} \\
-\sigma \mathrm{e}^{-i \theta_{\mathbf{k}}}
\end{array}\right) \quad|\sigma\rangle_{\mathbf{K}^{\prime}, \mathbf{k}}=\frac{1}{\sqrt{2}}\left(\begin{array}{c}
\mathrm{e}^{-i \theta_{\mathbf{k}}} \\
-\sigma \mathrm{e}^{i \theta_{\mathbf{k}}}
\end{array}\right) .
$$

The direction of the pseudo-spin eigenstates in BLG have a conceptually more difficult relationship with the direction of momentum than SLG. However, the pseudospin direction for an eigenstate can be written as

$$
\mathbf{S}_{\mathbf{K}, \mathbf{k}, \sigma}^{(\mathrm{blg})}=-\sigma\left(\cos 2 \theta_{\mathbf{k}},-\sin 2 \theta_{\mathbf{k}}, 0\right), \quad \mathbf{S}_{\mathbf{K}^{\prime}, \mathbf{k}, \sigma}^{(\mathrm{blg})}=-\sigma\left(\cos 2 \theta_{\mathbf{k}}, \sin 2 \theta_{\mathbf{k}}, 0\right) .
$$

The positive (negative) pseudo-spin eigenstate points anti-parallel (parallel) to the angle $-2 \theta_{\mathbf{k}}$ in the $\mathbf{K}$ valley, and anti-parallel (parallel) to the angle $+2 \theta_{\mathbf{k}}$ at the $\mathbf{K}$ valley. $\mathbf{S}_{\gamma, \mathbf{k}, \sigma}^{(\mathrm{blg})}$ is shown in Fig. 2.4. where it is compared to the direction of momentum. 


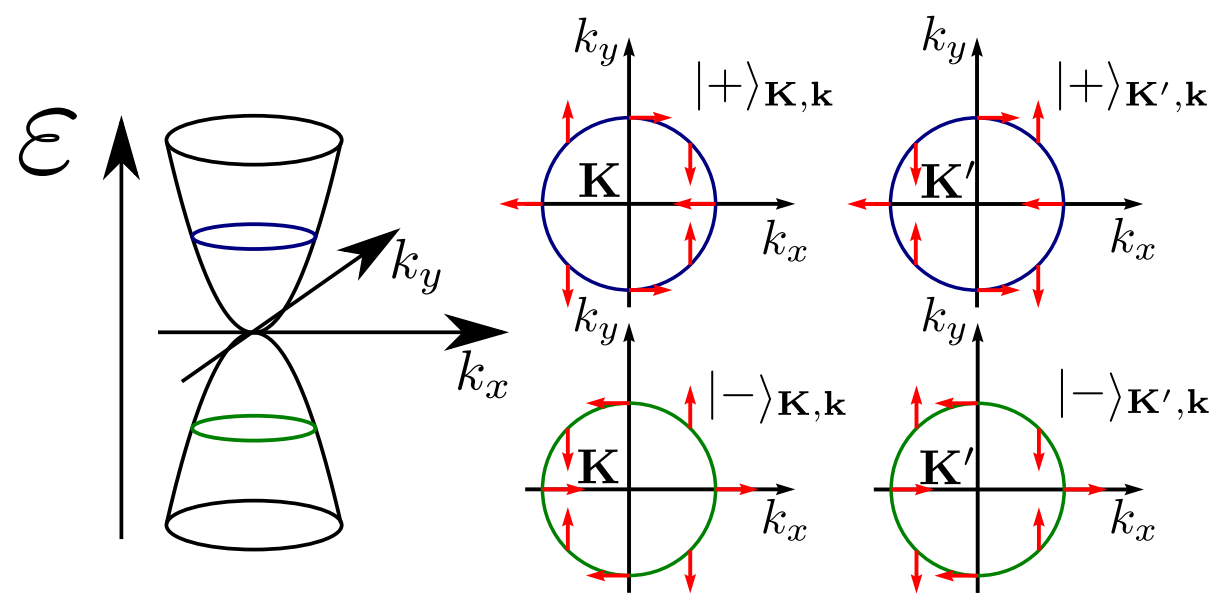

Figure 2.4: On the left is the dispersion relation at the $\mathbf{K}$ or $\mathbf{K}^{\prime}$ valley of BLG. On the right slices of the two touching parabola for the conduction (blue) and valence (green) band for the $\mathbf{K}$ and $\mathbf{K}^{\prime}$ valleys. The red arrows show the direction of the pseudo-spin eigenstate $|\sigma\rangle_{\gamma, \mathbf{k}}$, relative to the direction of momentum. For the $\mathbf{K}$ valley, the positive (negative) pseudo-spin eigenstates point anti-parallel (parallel) to $-2 \theta_{\mathbf{k}}$, where $\theta_{\mathbf{k}}$ is the angle of momentum. The positive (negative) pseudo-spin eigenstate in the $\mathbf{K}^{\prime}$ valley points anti-parallel (parallel) to the angle $+2 \theta_{\mathbf{k}}$.

\subsection{Molybdenum disulphide}

The lattice structure of $\mathrm{MoS}_{2}$ can be seen in Fig. 2.5, where it is shown to be a honeycomb structure with sulphur and molybdenum atoms. Each valley in $\mathrm{MoS}_{2}$ has Hamiltonian similar to the two-dimensional Dirac equation for a massive particle [50, 51,

$$
\mathcal{H}_{\mathbf{K}}^{(\mathrm{mos})}=v \hbar\left(\begin{array}{cc}
k_{\Delta} & k \mathrm{e}^{-i \theta_{\mathbf{k}}} \\
k \mathrm{e}^{i \theta_{\mathbf{k}}} & k_{\Delta}
\end{array}\right) \quad \text { and } \quad \mathcal{H}_{\mathbf{K}^{\prime}}^{(\mathrm{mos})}=v \hbar\left(\begin{array}{cc}
k_{\Delta} & k \mathrm{e}^{-i\left(\pi-\theta_{\mathbf{k}}\right)} \\
k \mathrm{e}^{i\left(\pi-\theta_{\mathbf{k}}\right)} & k_{\Delta}
\end{array}\right)
$$

where $v \hbar k_{\Delta}$ is the band gap energy $(1.2 \mathrm{eV}) \cdot \mathrm{MoS}_{2}$ has the energy-momentum relation

$$
\varepsilon_{\gamma, \mathbf{k}, \sigma}^{(\operatorname{mos})}=\sigma v \hbar \sqrt{k^{2}+k_{\Delta}^{2}}
$$

and the pseudo-spin eigenstates for each valley are

$$
|\sigma\rangle_{\mathbf{K}, \mathbf{k}}^{(\operatorname{mos})}=\frac{1}{\sqrt{2}}\left(\begin{array}{c}
\sqrt{\frac{1+\sigma \zeta_{\mathbf{k}}}{2}} \mathrm{e}^{-i \theta_{\mathbf{k}} / 2} \\
\sigma \sqrt{\frac{1-\sigma \zeta_{\mathbf{k}}}{2}} \mathrm{e}^{i \theta_{\mathbf{k}} / 2}
\end{array}\right) \quad|\sigma\rangle_{\mathbf{K}^{\prime}, \mathbf{k}}^{(\mathrm{mos})}=\frac{1}{\sqrt{2}}\left(\begin{array}{c}
\sqrt{\frac{1+\sigma \zeta_{\mathbf{k}}}{2}} \mathrm{e}^{-i\left(\pi-\theta_{\mathbf{k}}\right) / 2} \\
\sigma \sqrt{\frac{1-\sigma \zeta_{\mathbf{k}}}{2}} \mathrm{e}^{i\left(\pi-\theta_{\mathbf{k}}\right) / 2}
\end{array}\right)
$$

where $\zeta_{\mathbf{k}}=k_{\Delta} / \sqrt{k^{2}+k_{\Delta}^{2}}$. For large values of momentum $\zeta_{\mathbf{k}} \approx 0$, while for small values $\zeta_{\mathrm{k}} \approx 1$. The average pseudo-spin for each eigenstate is

$$
\mathbf{S}_{\mathbf{K}, \mathbf{k}, \sigma}^{(\operatorname{mos})}=\sigma\left(\sqrt{1-\zeta_{\mathbf{k}}^{2}} \cos \theta_{\mathbf{k}}, \sqrt{1-\zeta_{\mathbf{k}}^{2}} \sin \theta_{\mathbf{k}}, \zeta_{\mathbf{k}}\right)
$$



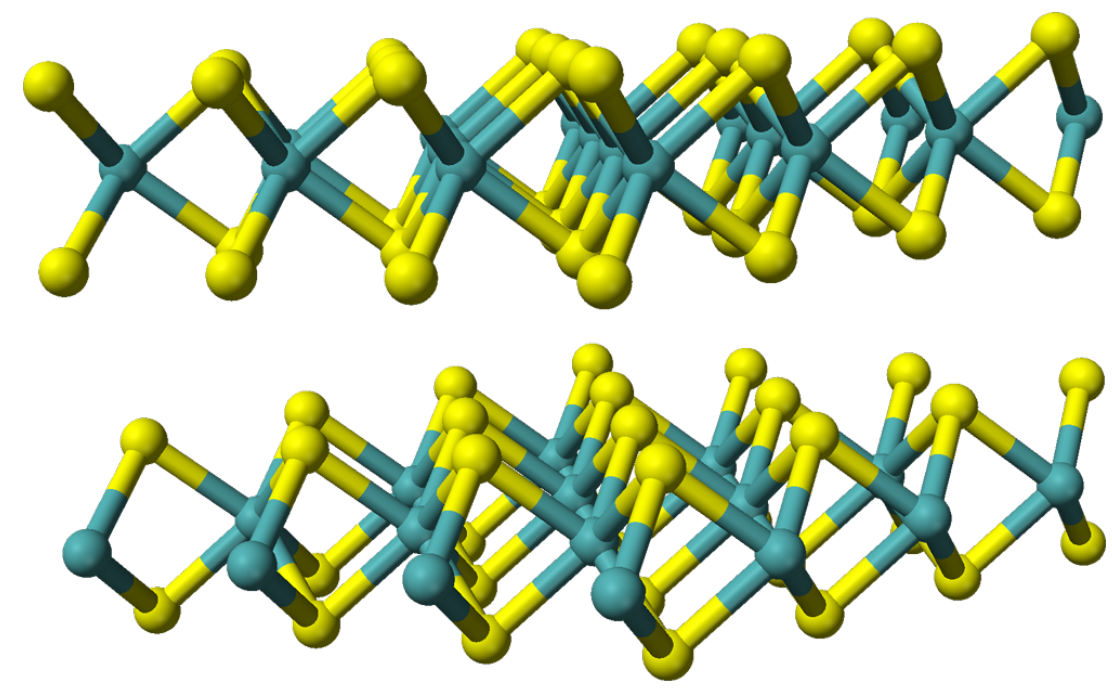

Figure 2.5: The lattice structure of $\mathrm{MoS}_{2}$ from two points of view, with sulphur atoms in yellow and molybdenum atoms in teal. This image has been taken from Ref. [49].

and

$$
\mathbf{S}_{\mathbf{K}^{\prime}, \mathbf{k}, \sigma}^{(\operatorname{mos})}=\sigma\left(-\sqrt{1-\zeta_{\mathbf{k}}^{2}} \cos \theta_{\mathbf{k}}, \sqrt{1-\zeta_{\mathbf{k}}^{2}} \sin \theta_{\mathbf{k}}, \zeta_{\mathbf{k}}\right)
$$

At zero momentum $\zeta_{\mathbf{k}}=1$, and the pseudo-spin is polarized along the $z$-axis. As the momentum increases, the pseudo-spin direction is pushed into the $x y$-plane, with $\zeta_{\mathbf{k}} \rightarrow 0$. When $k$ is much larger than $k_{\Delta}, \mathrm{MoS}_{2}$ has similar pseudo-spin eigenstates to SLG.

\subsection{Carrier concentration and density of states at the Fermi energy}

In this section, we present the electron density $n$ and density of states at the Fermi energy $\rho_{\mathrm{F}}$ at zero temperature, for SLG, BLG, and $\mathrm{MoS}_{2}$ (see Tab. 2.1).

To calculate the electron density [52], we sum all momentum states within a Fermi circle to obtain

$$
n=g_{\mathrm{s}} g_{\mathrm{v}} \frac{\pi k_{\mathrm{F}}^{2}}{(2 \pi)^{2}}
$$

where we have included spin and valley degeneracies $g_{\mathrm{s}}$ and $g_{\mathrm{v}}$.

The density of states $\rho(\varepsilon)$ is defined as

$$
\rho(\varepsilon)=\frac{\mathrm{d} N(\varepsilon)}{\mathrm{d} \varepsilon}
$$


Table 2.1: A list of the carrier concentration $n$ and density of states $\rho_{\mathrm{F}}$ at the Fermi energy, at zero temperature. $\rho_{\mathrm{F}}$ does not include spin, pseudo-spin, or valley degeneracy.

\begin{tabular}{|c|c|c|}
\hline & $n$ & $\rho_{\mathrm{F}}$ \\
\hline SLG & $\frac{1}{\pi(v \hbar)^{2}} \varepsilon_{\mathrm{F}}^{2}$ & $\frac{1}{2 \pi(v \hbar)^{2}} \varepsilon_{\mathrm{F}}$ \\
\hline BLG & $\frac{2 M}{\hbar^{2}} \varepsilon_{\mathrm{F}}$ & $\frac{M}{2 \pi \hbar^{2}}$ \\
\hline $\mathrm{MoS}_{2}$ & $\frac{1}{\pi(v \hbar)^{2}}\left(\varepsilon_{\mathrm{F}}^{2}-\Delta^{2}\right)$ & $\frac{1}{2 \pi(v \hbar)^{2}} \varepsilon_{\mathrm{F}}$ \\
\hline
\end{tabular}

where $N(\varepsilon)$ is the number of states at energy $\varepsilon$ per a unit volume [52]. To calculate the density of states at the Fermi energy, one can use the single particle retarded Green's function for a time independent and transnationally invariant system

$$
\mathcal{G}_{\alpha}^{\mathrm{R}}(t, \mathbf{k})=-i \Theta(t)\left\langle\left\{c_{\alpha \mathbf{k}}(t), c_{\alpha \mathbf{k}}^{\dagger}(0)\right\}\right\rangle,
$$

which is the phase and amplitude for a particle to propagate between states $(\mathbf{k}, \alpha) \rightarrow$ $(\mathbf{k}, \beta)$ in time $t . c_{\alpha \mathbf{k}}$ and $c_{\alpha \mathbf{k}}^{\dagger}$ are creation and annihilation operators. We have used the notation $\langle\ldots\rangle=\operatorname{Tr}[\ldots \hat{\rho}]$ as the thermal average, where $\hat{\rho}$ is the density matrix for the system.

This allows one to calculate the spectral function

$$
\mathcal{A}_{\alpha}(\varepsilon, \mathbf{k})=-2 \operatorname{Im} \mathcal{G}_{\alpha}^{\mathrm{R}}(\varepsilon, \mathbf{k}),
$$

from the Fourier transform of the single particle retarded Green's function. The spectral function is a normalised weighting of possible excitations in a system, indicating if it is possible for a particle to be in state $(\alpha, \mathbf{k})$ with energy $\varepsilon$ [53]. If we consider the density of states at the Fermi energy with no spin or pseudo-spin degeneracy

$$
\rho_{\mathrm{F}} \equiv \rho\left(\varepsilon_{\mathrm{F}}\right)=\frac{1}{2 \pi} \frac{1}{(2 \pi)^{2}} \int \mathrm{d}^{2} k \mathcal{A}\left(\varepsilon_{\mathrm{F}}, \mathbf{k}\right) .
$$

When there are no interactions, the spectral function will only allow excitations that follow the band structure $\mathcal{A}(\varepsilon, \mathbf{k})=2 \pi \delta\left(\varepsilon-\varepsilon_{\mathbf{k}}\right)$ [53]. 


\section{Chapter 3}

\section{Theory of magneto-tunnelling trans- port}

In this chapter, we review and present standard theory of magneto-tunnelling transport. This starts with using linear response theory to derive a formula for tunnelling current and conductance. Then we review past work, where magneto-tunnelling transport between two parallel two-dimensional electron gases was studied. These works are important, as the basis of this thesis is to apply these tools to investigate two-dimensional chiral electron gases.

\subsection{Linear response theory for tunnelling trans- port}

A full derivation of the tunnelling current shown here can be found in Ref. [53]. We follow a similar format. To model two coupled systems we use the single particle Hamiltonian

$$
\mathcal{H}=\left(\begin{array}{cc}
\mathcal{H}^{(1)} & \mathcal{T} \\
\mathcal{T}^{\dagger} & \mathcal{H}^{(2)}
\end{array}\right)
$$

One can use this to write a many body Hamiltonian in terms of single particle eigenstates, with

$$
\mathcal{H}^{(m)}=\sum_{\alpha} \varepsilon_{\alpha}^{(m)} c_{m, \alpha}^{\dagger} c_{m, \alpha}
$$

as the Hamiltonian for system $m=1,2$, with energies $\varepsilon_{m, \alpha} \cdot c_{m, \alpha}$ and $c_{m, \alpha}^{\dagger}$ are the creation and annihilation operators for system $m$, and $\alpha$ is the single particle eigenstate. To couple the two systems across the barrier we use the tunnelling Hamiltonian

$$
\mathcal{T}=\sum_{\nu \eta}\left\langle\psi_{\nu}^{(1)}|\mathcal{T}| \psi_{\eta}^{(2)}\right\rangle c_{1, \nu}^{\dagger} c_{2, \eta}+\text { H.C. }
$$

where $\left\langle\psi_{\nu}^{(1)}|\mathcal{T}| \psi_{\eta}^{(2)}\right\rangle$ is the tunnelling matrix element between the single particle eigenstates $\left|\psi_{\eta}^{(2)}\right\rangle$ and $\left|\psi_{\nu}^{(1)}\right\rangle$. To include a chemical potential in each system we add the term

$$
\mu^{(m)} \hat{N}^{(m)}
$$


where $\hat{N}=\sum_{\alpha} c_{\alpha}^{\dagger} c_{\alpha}$ is the operator for the total number of particles. Collecting all terms, we find

$$
\mathcal{H}=\mathcal{H}^{(1)}+\mathcal{H}^{(2)}+\mathcal{T}-\mu^{(1)} \hat{N}^{(1)}-\mu^{(2)} \hat{N}^{(2)}
$$

One can perform a unitary transformation to obtain the equivalent Hamiltonian

$$
\overline{\mathcal{H}}=\mathcal{H}^{(1)}+\mathcal{H}^{(2)}+\overline{\mathcal{T}}
$$

where

$$
\overline{\mathcal{T}}=\sum_{\nu \eta}\left\langle\psi_{\nu}^{(1)}|\mathcal{T}| \psi_{\eta}^{(2)}\right\rangle c_{1, \nu}^{\dagger} c_{2, \eta} \mathrm{e}^{\frac{i}{\hbar}\left[\mu^{(1)}-\mu^{(2)}\right] t}+\text { H.C. }
$$

Using the Heisenberg representation, the current operator is found to be

$$
I(t)=-\frac{e}{\hbar} \sum_{\nu \eta}\left\langle\psi_{\nu}^{(1)}|\mathcal{T}| \psi_{\eta}^{(2)}\right\rangle c_{1, \nu}^{\dagger} c_{2, \eta} \mathrm{e}^{\frac{i}{\hbar}\left[\mu^{(1)}-\mu^{(2)}\right] t}-\text { H.C. }
$$

In the weak tunnelling limit, we can treat the coupling between our systems as a perturbation. From this perturbation, we can calculate the average tunnelling current using the Kubo formula from Linear response theory. If introducing the coupling between the systems is done adiabatically, the Kubo formula is

$$
\langle I(t)\rangle=\langle I\rangle_{0}-\frac{i}{\hbar} \int_{-\infty}^{\infty} \mathrm{d} t^{\prime} \Theta\left(t-t^{\prime}\right)\left\langle\left[I_{I}(t), \overline{\mathcal{T}}_{I}\left(t^{\prime}\right)\right]\right\rangle_{0}
$$

where $\langle\ldots\rangle_{0}$ denotes the equilibrium average, and $A_{I}(t)$ denotes an operator in the interaction representation. At equilibrium, the number of particles in each system is conserved, so $\langle I\rangle_{0}=0$.

Using Wick's theorem, particle number conservation at equilibrium, and then relating Green's functions to the spectral function, it can be shown that

$$
I(V)=\frac{e}{\hbar} \sum_{\alpha \beta} \int_{-\infty}^{\infty} \frac{\mathrm{d} \varepsilon}{2 \pi} \mathcal{A}_{\alpha}^{(1)}(\varepsilon)\left|\left\langle\psi_{\alpha}^{(1)}|\mathcal{T}| \psi_{\beta}^{(2)}\right\rangle\right|^{2} \mathcal{A}_{\beta}^{(2)}(\varepsilon-e V)\left[n_{\mathrm{F}}(\varepsilon-e V)-n_{\mathrm{F}}(\varepsilon)\right] .
$$

We have made the substitution $\mu^{(1)}-\mu^{(2)}=e V$, and $n_{\mathrm{F}}$ denotes the Fermi-Dirac distribution. The finite bias tunnelling conductance can then be caculated from

$$
G(V) \equiv \frac{\partial I(V)}{\partial V}
$$

It follows that the tunnelling conductance in the zero bias limit is [23]

$$
\left.G(0) \equiv \frac{\partial I(V)}{\partial V}\right|_{V=0}=\frac{\mathrm{e}^{2}}{\hbar} \sum_{\alpha \beta} \int_{-\infty}^{\infty} \frac{\mathrm{d} \varepsilon}{2 \pi} \mathcal{A}_{\alpha}^{(1)}(\varepsilon)\left|\left\langle\psi_{\alpha}^{(1)}|\mathcal{T}| \psi_{\beta}^{(2)}\right\rangle\right|^{2} \mathcal{A}_{\beta}^{(2)}(\varepsilon)\left[-\frac{\partial n_{\mathrm{F}}}{\partial \varepsilon}(\varepsilon)\right]
$$


We will typically call this the linear tunnelling conductance, because it is the linear order term in the Taylor series expansion, i.e.

$$
I(V) \approx G(0) V+\mathcal{O}\left(V^{2}\right) .
$$

Of course, this shows for $V \approx 0$ that one can use Ohm's law, and $G(0)$ is the proportionality constant between $I$ and $V$.

Motivated by the form of the linear conductance, in this work we choose to write the tunnelling current in the form

$$
I(V)=\frac{1}{e} \int_{-\infty}^{\infty} \mathrm{d} \varepsilon \tilde{G}(\varepsilon, V)\left[n_{\mathrm{F}}(\varepsilon-e V)-n_{\mathrm{F}}(\varepsilon)\right],
$$

where

$$
\tilde{G}(\varepsilon, V)=\sum_{\alpha \beta} \frac{e^{2}}{2 \pi \hbar} \mathcal{A}_{\alpha}^{(1)}(\varepsilon)\left|\left\langle\psi_{\alpha}^{(1)}|\mathcal{T}| \psi_{\beta}^{(2)}\right\rangle\right|^{2} \mathcal{A}_{\beta}^{(2)}(\varepsilon-e V) .
$$

Now we will introduce some assumptions to simplify the tunnelling current and conductance. At zero temperature the Fermi-Dirac distribution becomes a step function, and it follows that

$$
\left[-\frac{\partial n_{\mathrm{F}}}{\partial \varepsilon}(\varepsilon)\right]=\delta\left(\varepsilon_{\mathrm{F}}-\varepsilon\right)
$$

Using the Hamiltonians in Eq. (3.2), which do not include interactions or disorder, the spectral function (defined in Eq. (2.24) ) is calculated to be

$$
\mathcal{A}_{\alpha}^{(m)}(\varepsilon)=2 \pi \delta\left(\varepsilon-\varepsilon_{\alpha}^{(m)}\right) .
$$

In the case of zero temperature, no interactions, and no disorder, we find

$$
\tilde{G}(\varepsilon, V)=\frac{2 \pi e^{2}}{\hbar} \sum_{\alpha \beta} \delta\left(\varepsilon-\varepsilon_{\alpha}^{(1)}\right)\left|\left\langle\psi_{\alpha}^{(1)}|\mathcal{T}| \psi_{\beta}^{(2)}\right\rangle\right|^{2} \delta\left(\varepsilon-e V-\varepsilon_{\beta}^{(2)}\right)
$$

which can be used to calculate the tunnelling current

$$
I(V)=\frac{1}{e} \int_{\varepsilon_{\mathrm{F}}}^{\varepsilon_{\mathrm{F}}+e V} \mathrm{~d} \varepsilon \tilde{G}(\varepsilon, V) .
$$

In fact, one can also show that

$$
G(0)=\frac{2 \pi \mathrm{e}^{2}}{\hbar} \sum_{\alpha, \beta} \delta\left(\varepsilon_{\mathrm{F}}^{(1)}-\varepsilon_{\alpha}^{(1)}\right)\left|\left\langle\psi_{\alpha}^{(1)}|\mathcal{T}| \psi_{\beta}^{(2)}\right\rangle\right|^{2} \delta\left(\varepsilon_{\mathrm{F}}^{(2)}-\varepsilon_{\beta}^{(2)}\right),
$$

where one can see that for equal Fermi energies in each layer $G(0)=\tilde{G}\left(\varepsilon_{\mathrm{F}}, 0\right)$. We calculate Eq. (3.18) and Eq. (3.20) for magneto-tunnelling transport using the same methods. In chapter 6 of this work, we discuss how the two quantities are related. 

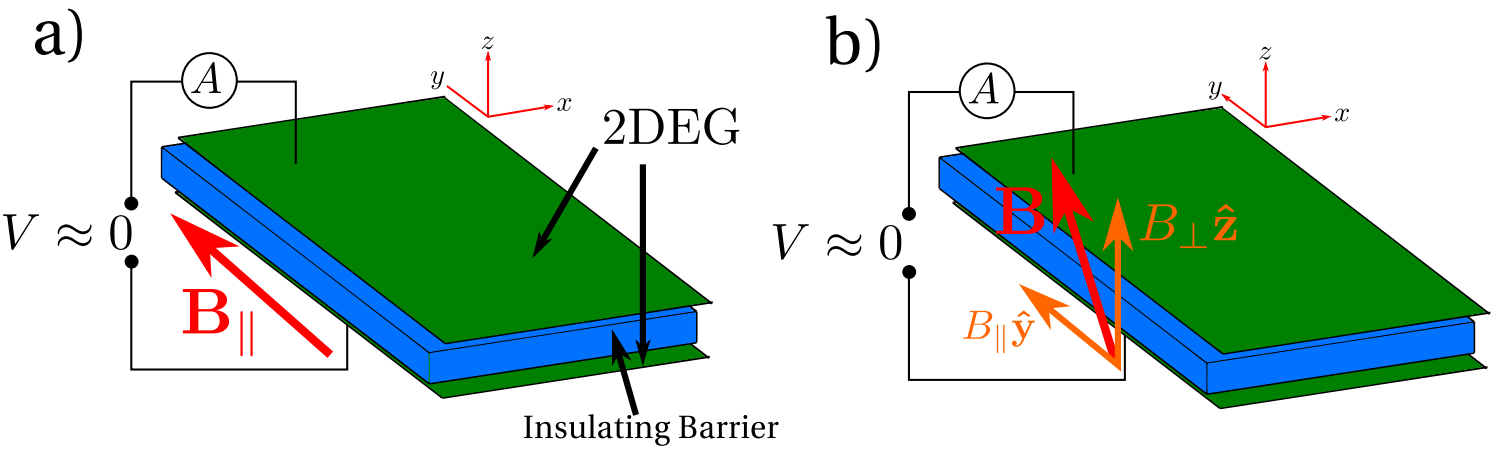

c)

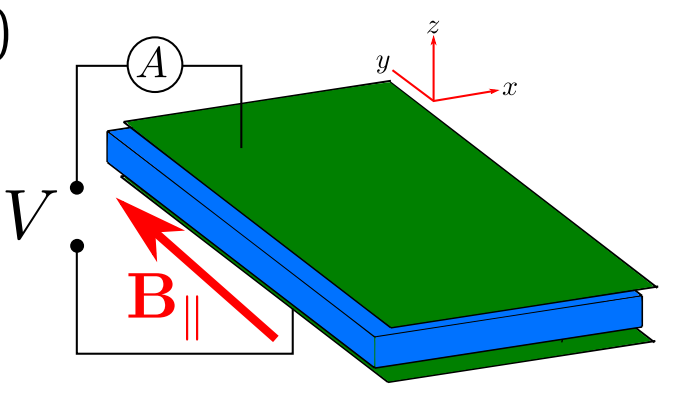

Figure 3.1: The double layer 2DEG systems reviewed in this section. Green represents the 2DEG systems, and blue is the insulating barrier. In this section, we review the magneto-tunnelling transport for a) an in-plane magnetic field with a small bias, b) a tilted magnetic field with a small bias, and c) an in-plane magnetic field with a finite bias. For a) \& b), we calculate the linear magneto-tunnelling conductance. While for c), we calculate the non-linear magneto-tunnelling current and conductance.

\subsection{Tunnelling between two-dimensional non-chiral electron gases}

In this section, we discuss and derive the linear magneto-tunnelling conductance between two parallel two-dimensional (non-chiral) electron gases, for an in-plane and tilted magnetic field. Then we discuss the finite bias magneto-tunnelling conductance between two parallel two-dimensional electron gases for an in-plane magnetic field. Each double layer system is shown in Fig. 3.1. First we list our assumptions for calculating Eq. 3.20). An ordinary two-dimensional electron gas (2DEG) has the energy-momentum relation

$$
\varepsilon_{\mathbf{k}}=\frac{\hbar^{2} k^{2}}{2 M}
$$

with eigenstates $|\mathbf{k}\rangle$. It is reasonable to assume that the barrier is homogeneous and that it conserves momentum [22 24], so the tunnelling matrix is of the form

$$
\mathcal{T}=\sum_{\mathbf{k}}|\mathbf{k}\rangle\langle\mathbf{k}| \tau_{0}
$$


where $\tau_{0}$ is a constant. Given that there are no magnetic impurities in the barrier, spin will be conserved in the tunnelling process. This allows one to sum over spin as a degeneracy $g_{\mathrm{s}}=2$. The last assumption is the continuum limit for summing over momentum states

$$
\sum_{\mathbf{k}} \rightarrow \frac{A}{(2 \pi)^{2}} \int \mathrm{d}^{2} k
$$

where $A$ denotes the area of a sample.

\subsubsection{The effect of an in-plane magnetic field}

Here we review work performed in Ref. [23], to derive the linear magneto-tunnelling conductance for an in-plane magnetic field $\mathbf{B}_{\|}=B_{\|} \hat{\mathbf{b}}_{\|}$. We use a vector potential of the form $\mathbf{A}=B_{\|} z \hat{\mathbf{b}}_{\|} \times \hat{\mathbf{z}}$. $\hat{\mathbf{b}}_{\|}$is the unit vector in the direction of the in-plane magnetic field. This means we can write our kinetic momentum in each layer as

$$
\Pi^{(m)}\left(\mathbf{k}, \mathbf{B}_{\|}\right)=\hbar \mathbf{k}+\hbar \frac{z_{m}}{\ell_{B_{\|}}^{2}} \hat{\mathbf{b}}_{\|} \times \hat{\mathbf{z}}
$$

where $\ell_{B_{\|}}=\sqrt{\frac{\hbar}{e B_{\|}}}$is the magnetic length and $z_{m} \hat{\mathbf{z}}$ is the location of system $m$. We find that the magnetic field shifts the kinetic momentum in each system by $-\hbar z_{m} / \ell_{B_{\|}}^{2} \hat{\mathbf{b}}_{\|} \times \hat{\mathbf{z}}$.

The energy for each system is

$$
\varepsilon_{\mathbf{k}}^{(m)}=\frac{\hbar^{2}}{2 M}\left\|\mathbf{k}+\frac{z_{m}}{\ell_{B_{\|}}^{2}} \hat{\mathbf{b}}_{\|} \times \hat{\mathbf{z}}\right\|^{2},
$$

with eigenstates $\left|\psi_{\mathbf{k}}\right\rangle=|\mathbf{k}\rangle$. One can then compute the linear magneto-tunnelling conductance

$$
G(0)=\frac{g_{\mathrm{s}} A\left|\tau_{0}\right|^{2}}{(2 \pi)^{2}} \frac{2 \pi \mathrm{e}^{2}}{\hbar} \int \mathrm{d}^{2} k \delta\left(\varepsilon_{\mathrm{F}}^{(1)}-\varepsilon_{\mathbf{k}}^{(1)}\right) \delta\left(\varepsilon_{\mathrm{F}}^{(2)}-\varepsilon_{\mathbf{k}}^{(2)}\right) .
$$

The possible momentum states at $\varepsilon_{\mathbf{k}}^{(m)}=\varepsilon_{\mathrm{F}}^{(m)}$ generate a shifted Fermi circle. The Fermi circles have been separated in kinetic momentum by $\mathbf{Q}=\left[\left(z_{2}-z_{1}\right) / \ell_{B_{\|}}^{2}\right] \hat{\mathbf{b}}_{\|} \times \hat{\mathbf{z}}$. Conservation of energy and momentum in a tunnelling event require that tunnelling can only occur between the momentum states at the points where the two Fermi circles intersect. This description is shown in Fig. 3.2.

We can evaluate the integral in Eq. (3.26), and we introduce the units of conductance

$$
G_{0}=\frac{g_{\mathrm{s}} g_{\mathrm{v}} \mathrm{e}^{2}}{2 \pi \hbar} \operatorname{Tr}\left[\tau^{\dagger} \tau\right] \frac{4 \pi^{2} \rho_{\mathrm{F}}^{(1)} \rho_{\mathrm{F}}^{(2)}}{k_{\mathrm{F}}^{(1)} k_{\mathrm{F}}^{(2)}} A .
$$

$\rho_{\mathrm{F}}$ is the density of states found in Table 2.1, and $g_{\mathrm{v}}=1$ is the valley degeneracy for a 2DEG. Presenting the conductance in units of $G_{0}$ allows us to remove structure due to the density of states, and obtain the structure due to the intersecting Fermi 


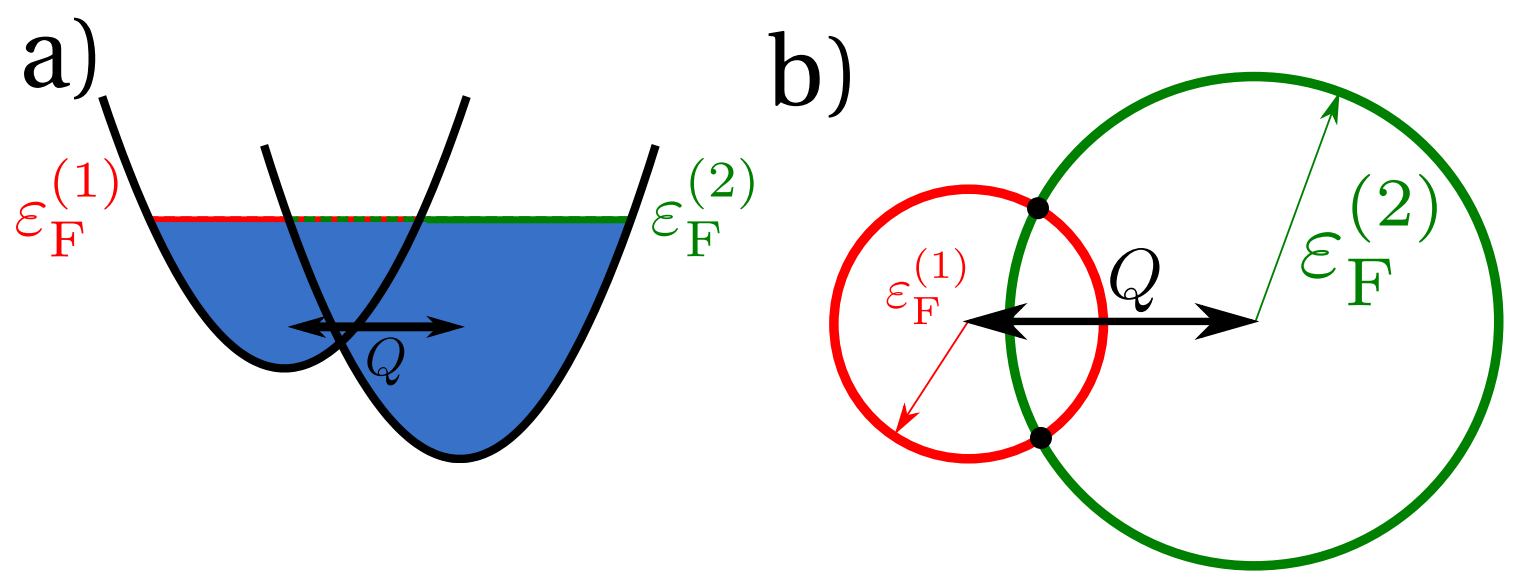

Figure 3.2: (a) Two energy-momentum curves for a 2DEG are separated by an inplane magnetic field. The separation in momentum space due to this magnetic field is $Q$. Since $V \approx 0$, tunnelling occurs at the Fermi energy for each system, where the Fermi energy is determined by the electron density in each layer. (b) Since tunnelling only occurs at the Fermi energy, the magneto-tunnelling conductance can be described by two intersecting Fermi circles, where a Fermi circle is all momentum states at the Fermi energy. These red and green circles can be seen as a cross-section of (a). The two black points of intersection are states that conserve energy and momentum in tunnelling, so determine when tunnelling can occur.

circles. One can inter-change between using the Fermi wave number and the electron density. However, we will present all results in terms of the parameters

$$
2 \tilde{k}_{\mathrm{F}}=k_{\mathrm{F}}^{(1)}+k_{\mathrm{F}}^{(2)} \quad \text { and } \quad \Delta=\left|k_{\mathrm{F}}^{(1)}-k_{\mathrm{F}}^{(2)}\right| .
$$

In general, the zero bias magneto-tunnelling conductance is a function of electron densities in each layer, and the strength of the applied magnetic field. Implicitly, this determines the three parameters $\tilde{k}_{\mathrm{F}}, \Delta$, and $Q$.

The linear magneto-tunnelling conductance is [23]

$$
\frac{G^{(2 \mathrm{deg})}(0)}{G_{0}}=\frac{4 \tilde{k}_{\mathrm{F}}^{2}-\Delta^{2}}{\sqrt{\left[4 \tilde{k}_{\mathrm{F}}^{2}-Q^{2}\right]\left[Q^{2}-\Delta^{2}\right]}} \Theta(Q-\Delta) \Theta\left(2 \bar{k}_{F}-Q\right) .
$$

For equal densities electron densities in each layer this simplifies

$$
\frac{G^{(2 \mathrm{deg})}(0)}{G_{0}}=\frac{4 \bar{k}_{\mathrm{F}}^{2}}{Q \sqrt{4 \bar{k}_{\mathrm{F}}^{2}-Q^{2}}} \Theta\left(2 \bar{k}_{\mathrm{F}}-Q\right)
$$

a plot for this equation is shown in Fig. 3.3, where it is compared with two intersecting Fermi circles. The magneto-tunnelling conductance shows a resonance when the Fermi circles overlap $(Q=\Delta)$ and when they are kissing $\left(Q=2 \bar{k}_{\mathrm{F}}\right)$. This result was first presented in the study in Ref. [23], where it was also computed with 


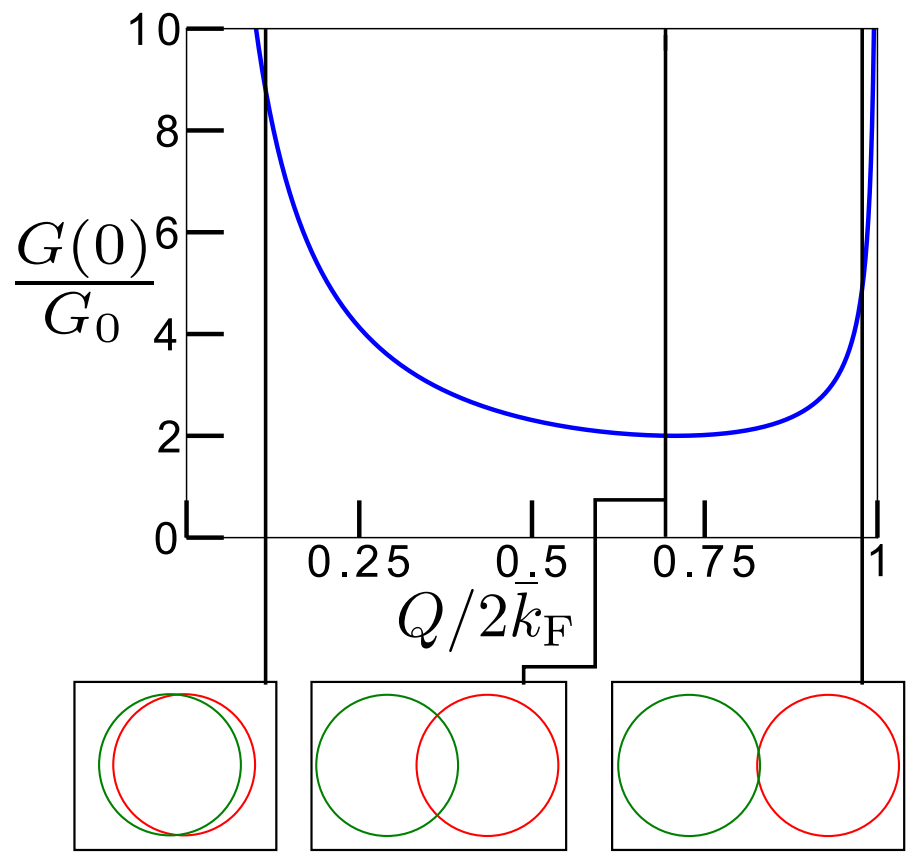

Figure 3.3: A plot of the linear magneto-tunnelling conductance for two parallel electron gases within an in-plane magnetic field. Each layer has equal electron densities $(\Delta=0)$, so this plot corresponds to Eq. 3.30 as a function of $Q$. As explained in Fig. 3.2 , the linear magneto-tunnelling conductance can be described by the points of two intersecting Fermi circles shown below the plot. The radius of each Fermi circle is $\bar{k}_{\mathrm{F}}$, and the separation between the circles is $Q$. The black lines across the plot, attached to the boxes, indicate the separation of the Fermi circles inside the boxes. One finds a resonance when the Fermi circles overlap $\left(Q / 2 \bar{k}_{\mathrm{F}}=0\right)$ and when they kiss $\left(Q / 2 \bar{k}_{\mathrm{F}}=1\right)$. These resonances indicate when the tunnelling current switches on. 
a finite electron life-time (the time for electrons scatter with impurities) in the two systems. When there is no disorder, the resonances diverge. But in the presence of disorder, the resonances have a finite peak and width of a Lorentzian determined by the electron life-time. This result was also shown to match the experiment performed in Ref. [30], and was used to measure the electron life-time in two parallel two-dimensional electron gases.

\subsubsection{The effect of a tilted magnetic field}

In this section, we calculate the linear magneto-tunnelling conductance when the magnetic field is titled in the $z y$ plane, i.e. $\mathbf{B}=B_{\|} \hat{\mathbf{y}}+B_{\perp} \hat{\mathbf{z}}$. This work was first performed in Ref. [24].

For definiteness, we choose the Landau gauge $\mathbf{A}=\left(-y B_{\perp}+z B_{\|}, 0,0\right)$, but the same results can be easily obtained from any other gauge. One can use the ladder operators $a^{ \pm}=\ell_{B_{\perp}}\left(\Pi_{x} \pm i \Pi_{y}\right) /(\sqrt{2} \hbar)$, which follow the commutation relation $\left[a^{-}, a^{+}\right]=1[54]$. We use the eigenstates $a^{+} a^{-}\left|\nu, k_{x}\right\rangle=\nu\left|\nu, k_{x}\right\rangle$. We can then write the Hamiltonian for a two-dimensional electron gas as

$$
\mathcal{H}=\frac{\hbar \Omega_{c}}{2}\left(a^{-} a^{+}+a^{+} a^{-}\right)
$$

where $\Omega_{c}=e B_{\perp} / M$ is the cyclotron orbit frequency. The eigenstates and energies of $\mathcal{H}^{(m)}$ for layer $m$ are $\left|\nu^{(m)}, \kappa_{x}^{(m)}\right\rangle$ and $\varepsilon_{\nu^{(m)}, k_{x}^{(m)}}=\hbar \Omega_{c}\left(\nu^{(m)}+\frac{1}{2}\right)$, where $\kappa_{x}^{(m)}=k_{x}+\frac{z_{m}}{\ell_{B_{\|}}}$ is a degeneracy that determines the cyclotron orbit guiding centre $\kappa_{x}^{(m)} \ell_{\perp}^{2}$. The linear magneto-tunnelling conductance for tunnelling between Landau levels is

$G(0)=\frac{g_{\mathrm{s}} \pi e^{2}\left|\tau_{0}\right|^{2}}{\hbar} \sum_{k_{x}} \sum_{\nu^{(1)}, \nu^{(2)}=0}^{\infty} \delta\left(\varepsilon_{\mathrm{F}}^{(1)}-\varepsilon_{\nu^{(1)}, k_{x}}^{(1)}\right)\left|\left\langle\nu^{(1)}, \kappa_{x}^{(1)} \mid \nu^{(2)}, \kappa_{x}^{(2)}\right\rangle\right|^{2} \delta\left(\varepsilon_{\mathrm{F}}^{(2)}-\varepsilon_{\nu^{(2)}, k_{x}}^{(2)}\right)$.

One can calculate the overlap between two Landau levels for two layers separated by distance $d=\left|z-z^{\prime}\right|$ (see Fig. 3.4)

$$
\left\langle\nu, \kappa_{x} \mid \nu^{\prime}, \kappa_{x}^{\prime}\right\rangle=\frac{\delta_{k_{x}, k_{x}^{\prime}} \mathrm{e}^{-\left(\frac{d \ell_{\perp}}{2 \ell_{\|}^{2}}\right)^{2}}}{\sqrt{2^{\nu} 2^{\nu^{\prime}} \nu^{\prime} ! \nu ! \pi}} \int_{-\infty}^{\infty} \mathrm{d} \eta \mathrm{e}^{-\eta^{2}} H_{\nu}\left(\eta+\frac{d \ell_{\perp}}{2 \ell_{\|}^{2}}\right) H_{\nu^{\prime}}\left(\eta-\frac{d \ell_{\perp}}{2 \ell_{\|}^{2}}\right),
$$

where $H_{\nu}(\cdot)$ is a Hermite polynomial. The above expression can be simplified further [24, 55]

$$
\left\langle\nu, \kappa_{x} \mid \nu^{\prime}, \kappa_{x}^{\prime}\right\rangle=\delta_{k_{x}, k_{x}^{\prime}}(-1)^{\nu_{>}-\nu_{<}}\left(\frac{\nu_{<} !}{\nu_{>} !}\right)^{\frac{1}{2}}\left(\frac{\xi^{2}}{2}\right)^{\frac{\nu_{>}-\nu_{<}}{2}} \mathrm{e}^{-\frac{\xi^{2}}{4}} L_{\nu<}^{\nu_{>}-\nu_{<}}\left(\frac{\xi^{2}}{2}\right)
$$

where $\nu_{<(>)}=\min (\max )\left\{\nu, \nu^{\prime}\right\}, \xi=\left(d / \ell_{B_{\perp}}\right)\left(B_{\|} / B_{\perp}\right)$, and $L_{n}^{n^{\prime}}(\cdot)$ is the generalized Laguerre polynomial. We can use the fact that the number of orbits in a Landau 


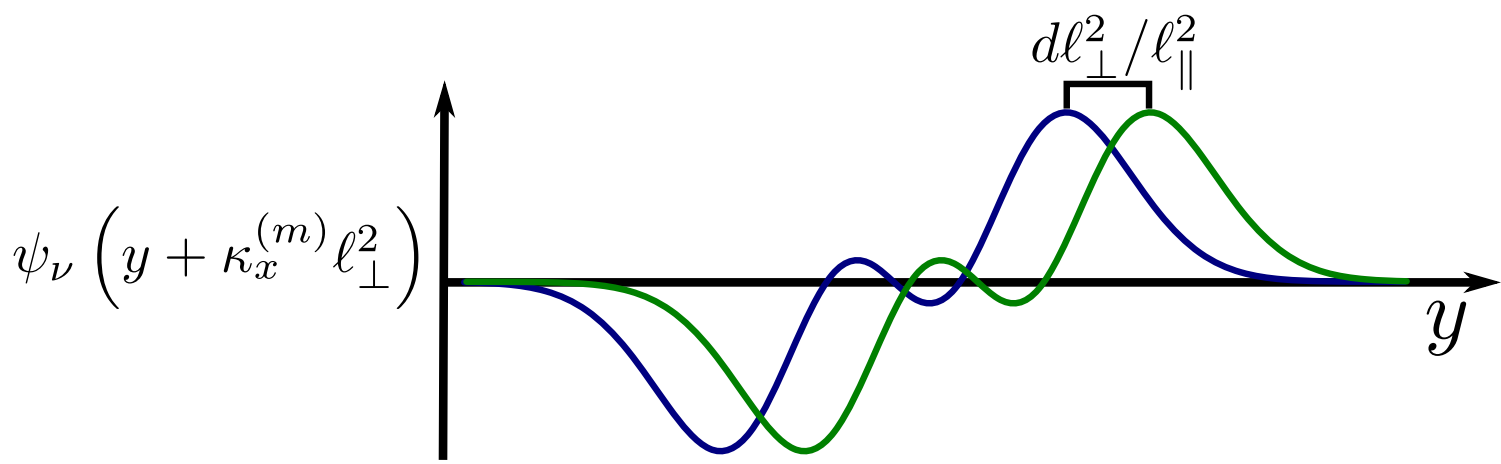

Figure 3.4: An example of two shifted Landau levels, due to $\mathbf{B}_{\|}$. The blue wave function is system 1 and the green wave function is system 2 . Increasing $\xi$ separates the Landau levels, creating oscillations in the wave function overlap $\left|\left\langle\nu^{(1)}, \kappa_{x}^{(1)} \mid \nu^{(2)}, \kappa_{x}^{(2)}\right\rangle\right|^{2}$.

level is limited by the size of the system

$$
\sum_{k_{x}} \approx \frac{L_{x}}{2 \pi} \int_{0}^{\frac{L_{y}}{\ell^{2}}}{ }^{2} \mathrm{~d} k_{x}=\frac{A}{2 \pi} \frac{e B_{\perp}}{\hbar}
$$

This leads to the magneto-tunnelling conductance

$G(0)=\frac{g_{\mathrm{s}} \pi e^{2}\left|\tau_{0}\right|^{2}}{\hbar} \frac{A}{2 \pi} \frac{M^{2}}{\hbar^{2}} \frac{1}{e B_{\perp} \hbar} \sum_{\nu^{(1)}, \nu^{(2)}=0}^{\infty} \delta\left(\nu_{\mathrm{F}}^{(1)}-\nu^{(1)}\right)\left|\left\langle\nu^{(1)}, \kappa_{x}^{(1)} \mid \nu^{(2)}, \kappa_{x}^{(2)}\right\rangle\right|^{2} \delta\left(\nu_{\mathrm{F}}^{(2)}-\nu^{(2)}\right)$.

Ref. [24] shows that for an in-plane magnetic field $\left(B_{\perp}=0\right)$, one obtains Eq. (3.29) as expected. For a perpendicular magnetic field $\left(B_{\|}=0\right)$, the linear magnetotunnelling conductance reduces to

$$
G(0)=\frac{g_{\mathrm{s}} \pi e^{2}\left|\tau_{0}\right|^{2}}{\hbar} \frac{A}{2 \pi} \frac{M^{2}}{\hbar^{2}} \frac{1}{e B_{\perp} \hbar} \sum_{\nu^{(1)}, \nu^{(2)}=0}^{\infty} \delta\left(\nu_{\mathrm{F}}^{(1)}-\nu^{(1)}\right) \delta\left(\nu_{\mathrm{F}}^{(2)}-\nu^{(2)}\right)
$$

\subsubsection{The effect of an in-plane magnetic field with a finite bias}

Now we will calculate the magneto-tunnelling current and conductance between two parallel two-dimensional electron gases in the presence of an in-plane magnetic field, when there is a finite bias applied between the two tunnel-coupled systems.

One should notice that the calculation of Eq. (3.18) is performed in the same way as the linear magneto-tunnelling conductance. For two parallel two-dimensional electron gases at equal densities, with an in-plane magnetic field and an applied bias 
between them, we can show that

$$
\tilde{G}^{(\text {ord })}(\varepsilon, V)=G_{0} \frac{4 \varepsilon_{\mathrm{F}}}{\sqrt{\hbar^{2} / 2 M} Q \sqrt{4 \varepsilon-\frac{\left[\left(\hbar^{2} / 2 M\right) Q^{2}+e V\right]^{2}}{\left(\hbar^{2} / 2 M\right) Q^{2}}}} \Theta\left(\varepsilon-\frac{\left[\left(\hbar^{2} / 2 M\right) Q^{2}+e V\right]^{2}}{4\left(\hbar^{2} / 2 M\right) Q^{2}}\right) .
$$

Using the relation $\varepsilon=\frac{\hbar^{2} k^{2}}{2 M}$, one can see the similarities with the zero bias conductance $G^{(\text {ord) }}(0)$ in Eq. 3.30 . In particular, the only difference is that the divergences are now modified as a function of the bias $e V$. The two formula are related because they both describe intersecting circles, where $\tilde{G}^{(\text {ord })}(\varepsilon, V)$ is determined by the intersecting cross-section at energy $\varepsilon$ of two energy-momentum relations separated in energy by $\mathrm{eV}$ and momentum $Q$.

Evaluating the integral in Eq. (3.19), one finds the magneto-tunnelling current as

$$
\begin{array}{r}
I^{(\text {ord })}(V)=\frac{G_{0}}{e} \frac{4 \varepsilon_{\mathrm{F}}}{2 \frac{\hbar}{\sqrt{2 M}} Q}\left[\Theta\left(4 \varepsilon_{\mathrm{F}}-\frac{\left(\frac{\hbar^{2}}{2 M} Q^{2}-e V\right)^{2}}{\frac{\hbar^{2}}{2 M} Q^{2}}\right) \sqrt{4 \varepsilon_{\mathrm{F}}-\frac{\left(\frac{\hbar^{2}}{2 M} Q^{2}-e V\right)^{2}}{\frac{\hbar^{2}}{2 M} Q^{2}}}\right. \\
-\Theta\left(4 \varepsilon_{\mathrm{F}}-\frac{\left(\frac{\hbar^{2}}{2 M} Q^{2}+e V\right)^{2}}{\frac{\hbar^{2}}{2 M} Q^{2}}\right) \sqrt{4 \varepsilon_{\mathrm{F}}-\frac{\left(\frac{\hbar^{2}}{2 M} Q^{2}+e V\right)^{2}}{\frac{\hbar^{2}}{2 M} Q^{2}}} .
\end{array}
$$

This formula is consistent with the expression for the magneto-tunnelling current found in [22]. One can differentiate with respect to $V$, and obtain the non-linear magneto-tunnelling conductance

$$
\begin{aligned}
& G^{(\text {ord })}(V)=G_{0}\left[\Theta\left(4 \varepsilon_{\mathrm{F}}-\frac{\left(\frac{\hbar^{2}}{2 M} Q^{2}-e V\right)^{2}}{\frac{\hbar^{2}}{2 M} Q^{2}}\right) \frac{4 \varepsilon_{\mathrm{F}} \frac{\left(\frac{\hbar^{2}}{2 M} Q^{2}-e V\right)}{2 \frac{\hbar^{2}}{2 M} Q^{2}}}{\frac{\hbar}{\sqrt{2 M}} Q \sqrt{4 \varepsilon_{\mathrm{F}}-\frac{\left(\frac{\hbar^{2}}{2 M} Q^{2}-e V\right)^{2}}{\frac{\hbar^{2}}{2 M} Q^{2}}}}\right. \\
& \left.+\Theta\left(4 \varepsilon_{\mathrm{F}}-\frac{\left(\frac{\hbar^{2}}{2 M} Q^{2}+e V\right)^{2}}{\frac{\hbar^{2}}{2 M} Q^{2}}\right) \frac{4 \varepsilon_{\mathrm{F}} \frac{\left(\frac{\hbar^{2}}{2 M} Q^{2}+e V\right)}{2 \frac{\hbar^{2}}{2 M} Q^{2}}}{\frac{\hbar}{\sqrt{2 M}} Q \sqrt{4 \varepsilon_{\mathrm{F}}-\frac{\left(\frac{\hbar^{2}}{2 M} Q^{2}+e V\right)^{2}}{\frac{\hbar^{2}}{2 M} Q^{2}}}}\right] .
\end{aligned}
$$

From this formula, it is clear that $G^{(\text {ord })}(V)=G^{(\text {ord })}(-V)$. One will find that $G^{(\text {ord })}(0)$ is consistent with Eq. (3.30). Also, one can use this formula to show that there are resonances at the locations

$$
\frac{e V}{\varepsilon_{\mathrm{F}}}=+\left(2 \pm \frac{Q}{k_{\mathrm{F}}}\right) \frac{Q}{k_{\mathrm{F}}} \text { and } \quad \frac{e V}{\varepsilon_{\mathrm{F}}}=-\left(2 \pm \frac{Q}{k_{\mathrm{F}}}\right) \frac{Q}{k_{\mathrm{F}}} .
$$

Fig. 3.5 shows a plot of the non-linear magneto-tunnelling conductance, where one can see these divergences create a fish shape. One can find that this plot is consistent with the plots of experimental measurements found in [41, 42], where the divergences follow the same fish shape and are discussed in detail. Fig. 3.6 explains how each resonance relates to the intersecting energy-momentum relations. 

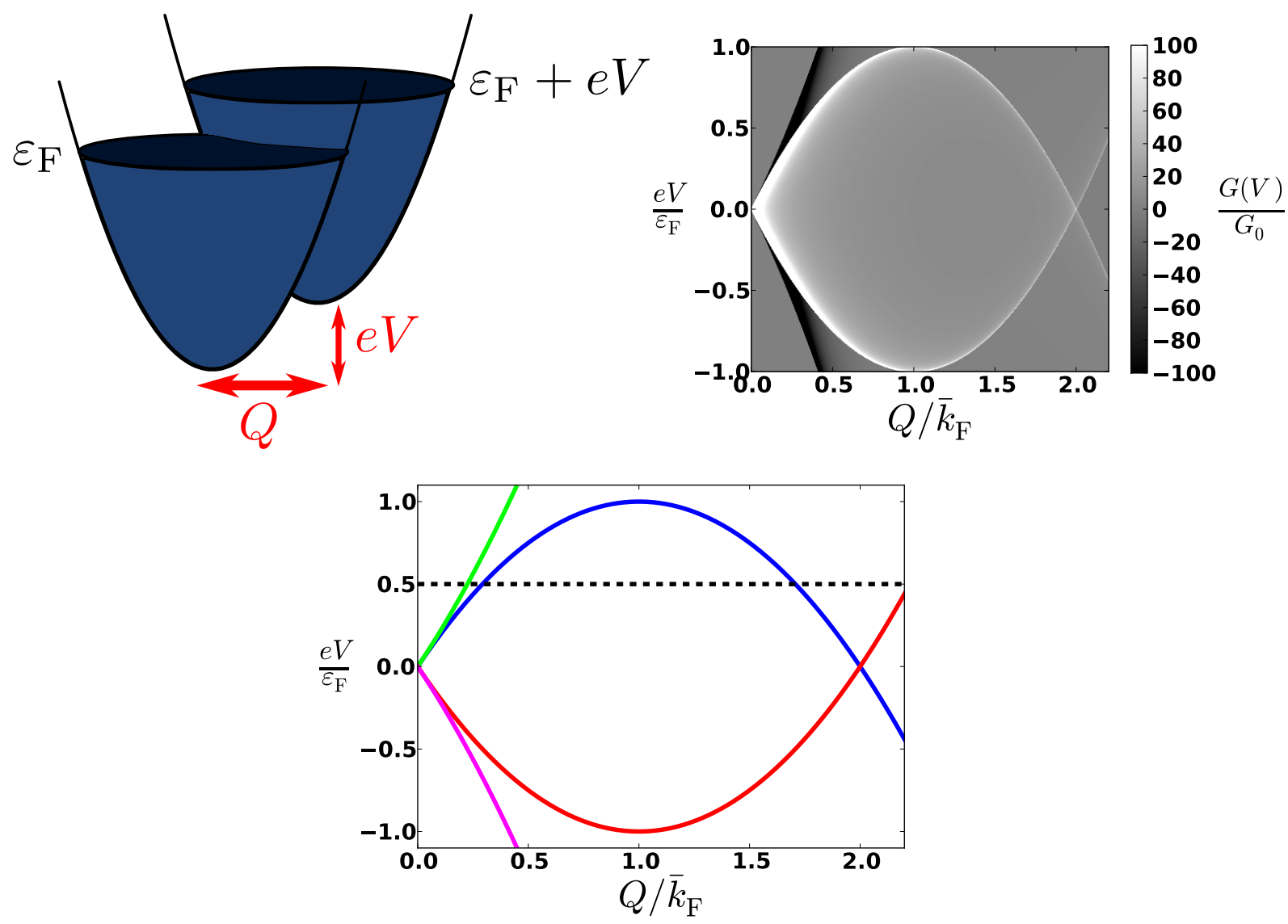

Figure 3.5: (Top Left) Two parabolic energy-momentum relations shifted, due to a magnetic field and bias, by momentum $Q$ and energy $e V$, where each system is occupied up to the Fermi level. (Top Right) A plot of the magneto-tunnelling conductance between two parallel 2DEG, as a function $Q$ and $e V$. The divergences create a distinct fish shape, which is present in experimental results from [41, 42]. (Bottom) One can see the lines of divergence $\frac{e V}{\varepsilon_{\mathrm{F}}}=\left(2 \pm \frac{Q}{k_{\mathrm{F}}}\right) \frac{Q}{k_{\mathrm{F}}}(+$ green, - blue) and $\frac{e V}{\varepsilon_{\mathrm{F}}}=-\left(2 \pm \frac{Q}{k_{\mathrm{F}}}\right) \frac{Q}{k_{\mathrm{F}}}(+$ pink, - red $)$. The dashed black line is a line of constant voltage, Fig. 3.6 shows the separated energy-momentum relations for the divergences along this line. 


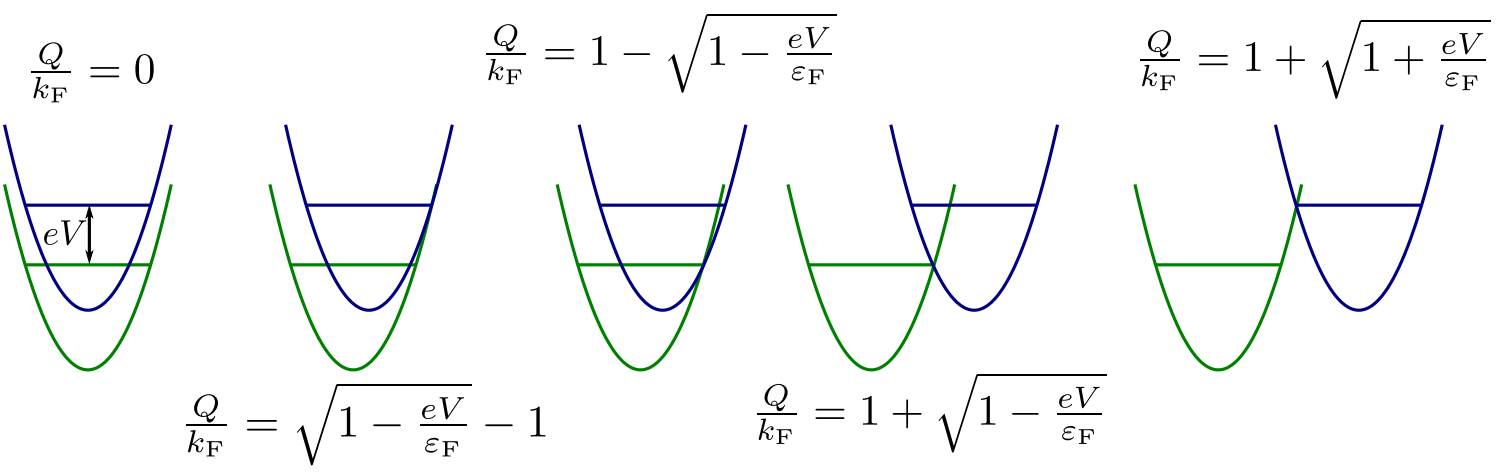

Figure 3.6: Two parabolic energy-momentum relations separated by energy $\mathrm{eV}$ and momentum $Q$, each diagram can be mapped to the divergences along the dashed black line in Fig. 3.5. Each system is occupied up to the Fermi energy represented by the line. At $Q=0$, no current can flow because the surfaces do not intersect. However, when the surfaces start to intersect at $\frac{Q}{k_{\mathrm{F}}}=\sqrt{1-\frac{e V}{\varepsilon_{\mathrm{F}}}}-1$ the current switches on. The current increases until $\frac{Q}{k_{\mathrm{F}}}=1-\sqrt{1-\frac{e V}{\varepsilon_{\mathrm{F}}}}$, because total intersection increases. At $\frac{Q}{k_{\mathrm{F}}}=1-\sqrt{1+\frac{e V}{\varepsilon_{\mathrm{F}}}}$ the current starts to decrease again, because the total intersection starts to decrease. The current switches off at $\frac{Q}{k_{\mathrm{F}}}=1+\sqrt{1+\frac{e V}{\varepsilon_{\mathrm{F}}}}$. 


\section{Chapter 4}

\section{Magneto-tunnelling between two di- mensional chiral electron gases}

In this chapter, we calculate the linear magneto-tunnelling conductance between two parallel two-dimensional chiral electron gases, extending previous work discussed in subsections 3.2.1 \& 3.2.2. We investigate magneto-tunnelling transport in a double layer van der Waals heterostructure. Specifically, we investigate the effect of an inplane magnetic field for SLG, BLG, and $\mathrm{MoS}_{2}$, and a tilted magnetic field in two parallel layers of SLG.

In order to look at the effect of chiral pseudo-spin states, we introduce the momentum-resolved pseudo-spin tunnelling matrix. This tunnelling matrix encodes the pseudo-spin properties of the barrier, which we discuss in section 5 .

For an in-plane magnetic field, we find that pseudo-spin overlap at the intersection of the Fermi circles dampens the tunnelling conductance as a function of $Q$. For a tilted magnetic field in SLG, we find oscillations in the linear magneto-tunnelling conductance as a function of $\xi=\left(d / \ell_{B_{\perp}}\right)\left(B_{\|} / B_{\perp}\right)$, and different oscillations are possible due to pseudo-spin couplings.

\subsection{Effect of an in-plane magnetic field}

In this section, we obtain an analytic formula for the linear magneto-tunnelling conductance between two parallel chiral systems, for an in-plane magnetic field (see Fig. 4.1). We introduce the assumptions and an outline for the calculation. We also introduce the chosen parametrisation of the momentum-resolved pseudo-spin tunnelling matrix. Then we compare calculations for SLG, BLG, and $\mathrm{MoS}_{2}$.

The possible states are determined by the valley $\gamma$, canonical momentum $\mathbf{k}$, and pseudo-spin $\sigma$. To calculate the linear tunnelling conductance, one can use Eq. 3.20 .

\subsubsection{Form of the tunnelling matrix}

To describe tunnelling between two systems, the systems are coupled with the tunnelling matrix $\mathcal{T}$. Thus, $\mathcal{T}$ encodes how particles can transition between the states of each system. The intrinsic spin of a particle is conserved in tunnelling, assuming there are no magnetic impurities in the barrier. However, we cannot ensure that 


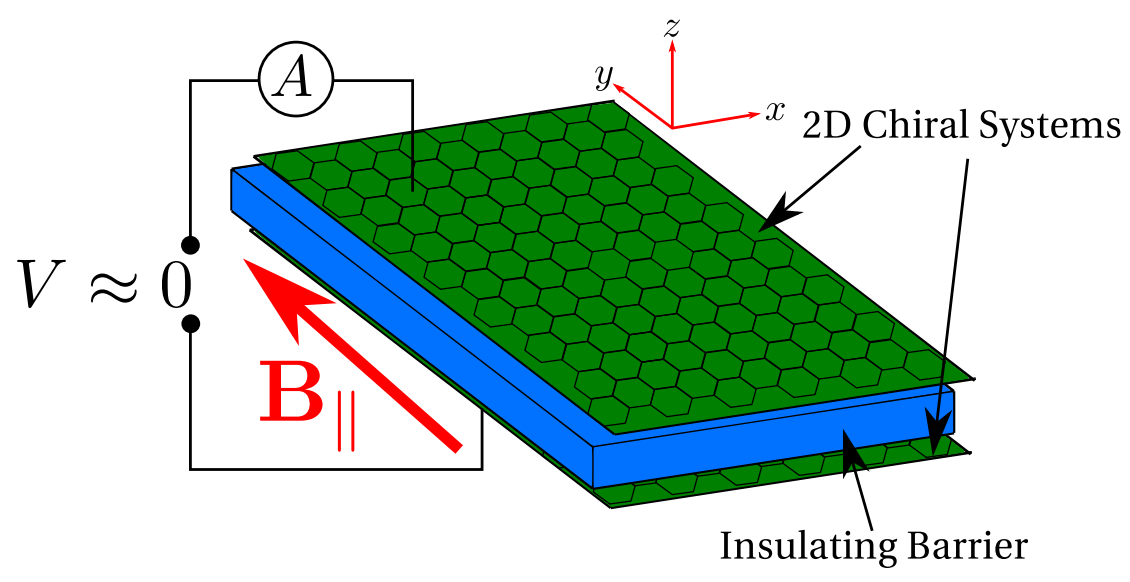

Figure 4.1: Two parallel two-dimensional chiral systems are separated by an atomically thick barrier. A small bias $V \approx 0$ is applied between the two layers. An in-plane magnetic field is applied along the barrier and two layers, as shown by the red arrow.

pseudo-spin is conserved in tunnelling, since it relies on the coupling between sublattices across the barrier. For two systems with pseudo-spin, the tunnelling matrix can be written as

$$
\mathcal{T}=\left(\begin{array}{ll}
\mathcal{T}_{\uparrow \uparrow} & \mathcal{T}_{\uparrow \downarrow} \\
\mathcal{T}_{\downarrow \uparrow} & \mathcal{T}_{\downarrow \downarrow}
\end{array}\right),
$$

where $\uparrow$ is spin pseudo-up and $\downarrow$ is pseudo-spin down along the $z$-axis. Each $\mathcal{T}_{\alpha \beta}$ is an operator that can be written in position or momentum representation.

When the barrier is translationally invariant, canonical momentum is conserved when a particle tunnels across the barrier [22 24]. This means a particle in momentum state $\left(k_{x}, k_{y}\right)$ can only tunnel to another state with the momentum $\left(k_{x}, k_{y}\right)$. Which suggests that the tunnelling matrix is diagonal in canonical momentum. Therefore, when momentum is conserved in tunnelling, the tunnelling matrix is

$$
\mathcal{T}=\sum_{\mathbf{k}}|\mathbf{k}\rangle\langle\mathbf{k}| \otimes \tau_{\mathbf{k}}
$$

$\tau_{\mathbf{k}}$ is a complex valued $2 \times 2$ matrix, it is the momentum-resolved pseudo-spin tunnelling matrix as a function of $\mathbf{k}$.

One can combine Eq. (2.3) and Eq. (4.2) to show that the tunnelling matrix element is

$$
\left\langle_{\gamma, \mathbf{k}^{(1)}, \sigma^{(1)}} \psi^{(1)}|\mathcal{T}| \psi_{\gamma, \mathbf{k}^{(2)}, \sigma^{(2)}}^{(2)}\right\rangle={ }_{\gamma, \boldsymbol{\Pi}^{(1)}}\left\langle\sigma^{(1)}\left|\tau_{\mathbf{k}}\right| \sigma^{(2)}\right\rangle_{\gamma, \boldsymbol{\Pi}^{(2)}}
$$

\subsubsection{Linear magneto-tunnelling conductance formula}

At zero temperature, with no interactions or disorder, and enforcing conservation of canonical momentum across the barrier, we can use Eq. 3.20 to calculate the linear 
magneto-tunnelling conductance

$G(0)=\left.\left.\frac{g_{\mathrm{s}} A}{(2 \pi)^{2}} \frac{2 \pi \mathrm{e}^{2}}{\hbar} \sum_{\gamma=\mathbf{K}, \mathbf{K}^{\prime}} \int \mathrm{d}^{2} k \delta\left(\varepsilon_{\mathrm{F}}^{(1)}-\varepsilon_{\sigma_{\mathrm{F}}^{(1)}, \mathbf{k}}^{(1)}\right)\right|_{\gamma, \boldsymbol{\Pi}^{(1)}}\left\langle\sigma_{\mathrm{F}}^{(1)}\left|\tau_{\mathbf{k}}\right| \sigma_{\mathrm{F}}^{(2)}\right\rangle_{\gamma, \boldsymbol{\Pi}^{(2)}}\right|^{2} \delta\left(\varepsilon_{\mathrm{F}}^{(2)}-\varepsilon_{\sigma_{\mathrm{F}}^{(2)}, \mathbf{k}}^{(2)}\right)$

where we have defined $\sigma_{\mathrm{F}}^{(m)}=\operatorname{Sign}\left(\varepsilon_{\mathrm{F}}^{(m)}\right)$. One can see this is the same as Eq. (3.26), but with the pseudo-spin tunnelling matrix element.

Due to the shift in kinetic momentum between the layers (see Eq. (3.24)), SLG, $\mathrm{BLG}$, and $\mathrm{MoS}_{2}$ have the dispersion relations

$$
\begin{gathered}
\varepsilon_{\gamma, \sigma, \mathbf{k}}^{\mathrm{slg},(m)}=\sigma v \hbar\left\|\mathbf{k}+\frac{z_{m}}{\ell_{B_{\|}}^{2}} \hat{\mathbf{b}}_{\|} \times \hat{\mathbf{z}}\right\|, \quad \frac{\varepsilon_{\gamma, \sigma, \mathbf{k}}^{\mathrm{blg},(m)}=\sigma \frac{\hbar^{2}}{2 M}\left\|\mathbf{k}+\frac{z_{m}}{\ell_{B_{\|}}^{2}} \hat{\mathbf{b}}_{\|} \times \hat{\mathbf{z}}\right\|^{2}}{\varepsilon_{\gamma, \sigma, \mathbf{k}}^{\mathrm{MoS}_{2},(m)}}=\sigma v \hbar \sqrt{\left\|\mathbf{k}+\frac{z_{m}}{\ell_{B_{\|}}^{2}} \hat{\mathbf{b}}_{\|} \times \hat{\mathbf{z}}\right\|^{2}+k_{\Delta}^{2}}
\end{gathered}
$$

In each type of chiral material, the possible momentum states for $\varepsilon_{\sigma, \mathbf{k}}^{(m)}=\varepsilon_{\mathrm{F}}^{(m)}$ generate a shifted Fermi circle. Even though SLG and $\mathrm{MoS}_{2}$ do not have parabollic energy-momentum relations, Fig. 4.2 explains how the linear magneto-tunnelling conductance can be viewed using two separated Fermi circles. As with an ordinary 2DEG, the Fermi circles have been separated in momentum space by $\mathbf{Q}=\left[\left(z_{2}-\right.\right.$ $\left.\left.z_{1}\right) / \ell_{B_{\|}}^{2}\right] \hat{\mathbf{b}}_{\|} \times \hat{\mathbf{z}}$. Conservation of energy and momentum in a tunnelling event require that tunnelling can only occur between the momentum states at the points where the two Fermi circles intersect. This means that any new characteristics in the linear magneto-tunnelling conductance will be due to pseudo-spin, and not due to the energy-momentum relation.

In this work, we assume that there is no tunnelling between the $\mathbf{K}$ and $\mathbf{K}^{\prime}$ valleys. For this assumption to be true requires that the valleys do not intersect. This is enforced when $Q<\frac{\pi}{a}$, where $a$ is the lattice constant. Letting $d=z_{2}-z_{1}$, one can write this as

$$
B_{\|}<\frac{\pi}{a} \frac{\hbar}{e d}
$$

One can assume that $a \approx 10^{-10}, d \approx 10^{-9}$, and one would need a magnetic field on the order of $10^{4} \mathrm{~T}$ to make the $\mathbf{K}$ and $\mathbf{K}^{\prime}$ valleys overlap.

One can show that the linear magneto-tunnelling conductance in the case of two intersecting Fermi circles is

$$
\begin{aligned}
& \frac{G(0)}{A}=\frac{g_{\mathrm{s}} \mathrm{e}^{2}}{\hbar} \sum_{\gamma} 2 \pi \rho_{\mathrm{F}}^{(1)} \rho_{\mathrm{F}}^{(2)}\left[\left|\Gamma_{\mathrm{u}}^{(\gamma)}\right|^{2}+\left|\Gamma_{\mathrm{l}}^{(\gamma)}\right|^{2}\right] \\
& \times \frac{\Theta\left(\|\mathbf{Q}\|-\left|k_{\mathrm{F}}^{(1)}-k_{\mathrm{F}}^{(2)}\right|\right) \Theta\left(k_{\mathrm{F}}^{(1)}+k_{\mathrm{F}}^{(2)}-\|\mathbf{Q}\|\right)}{\sqrt{\left[\left(k_{\mathrm{F}}^{(1)}+k_{\mathrm{F}}^{(2)}\right)^{2}-Q^{2}\right]\left[Q^{2}-\left(k_{\mathrm{F}}^{(1)}-k_{\mathrm{F}}^{(2)}\right)^{2}\right]}},
\end{aligned}
$$




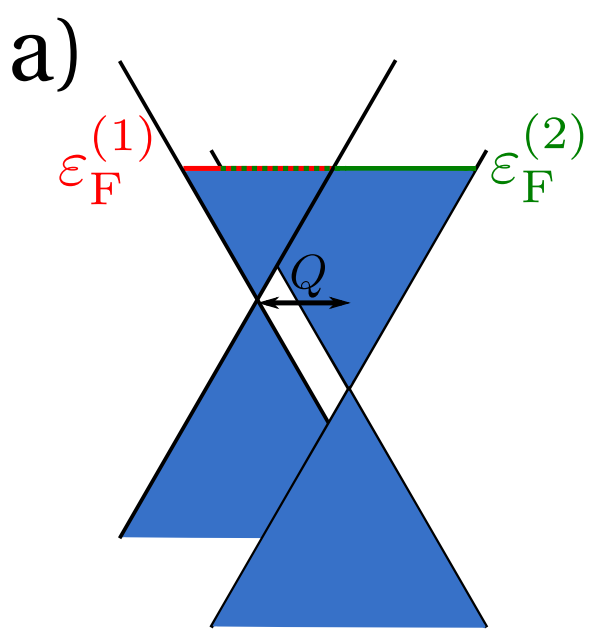

b)

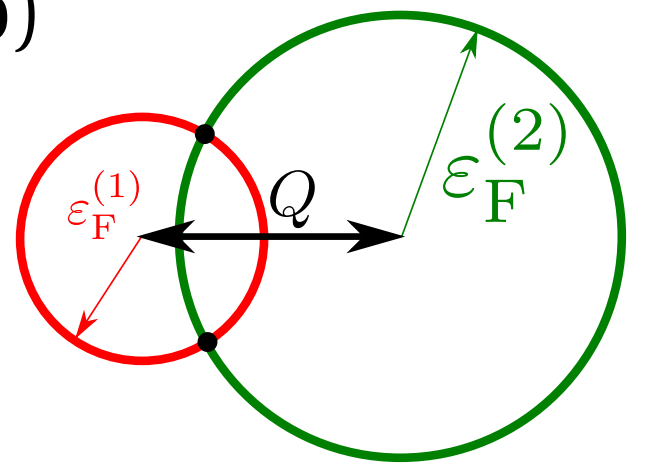

Figure 4.2: a) Diagram showing two linear energy-momentum relations separated in momentum space by distance $Q$. Each system has a different Fermi energy $\epsilon_{\mathrm{F}}^{(m)}$ due to different electron densities. This shows that even for a non-parabolic energymomentum relation, one can still view the tunnelling conductance as being explained by two intersecting Fermi circles. b) A diagram of two intersecting Fermi circles, separated in momentum space by distance $Q$. The colour of each Fermi circle relates to the conical cross-sections in a). The black dots at the intersections represent where tunnelling can occur, conserving energy and canonical momentum.

where

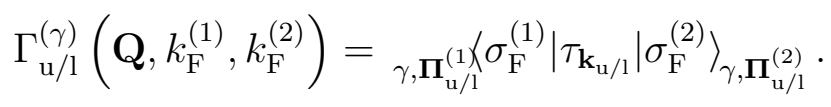

The density of states at the Fermi energy $\rho_{\mathrm{F}}$ does not include spin, and can be found in Table 2.1. We have defined $\sigma_{\mathrm{F}}^{(m)}=\operatorname{Sign}\left(\varepsilon_{\mathrm{F}}^{(m)}\right)$. We have defined $\mathbf{k}_{\mathrm{u} / 1}$ so that it represents where the Fermi circles intersect in canonical momentum space, and $u(l)$ is the upper (lower) intersection. In later results, we will assume that $\tau_{\mathbf{k}} \equiv \tau$.

We define the following

$$
\begin{aligned}
\left\|\boldsymbol{\Pi}_{\mathrm{u} / \mathrm{l}}^{(m)}\right\| & =\hbar k_{\mathrm{F}}^{(m)}, \\
\boldsymbol{\Pi}_{\mathrm{u} / 1}^{(1)}-\boldsymbol{\Pi}_{\mathrm{u} / \mathrm{l}}^{(2)} & =\hbar \mathbf{Q}, \\
\hbar \mathbf{k}_{\mathrm{u} / \mathrm{l}} & =\frac{1}{2}\left(\boldsymbol{\Pi}_{\mathrm{u} / \mathrm{l}}^{(1)}+\boldsymbol{\Pi}_{\mathrm{u} / \mathrm{l}}^{(2)}-\hbar \frac{z_{1}+z_{2}}{\ell_{B_{\|}}^{2}} \hat{\mathbf{b}} \times \hat{\mathbf{z}}\right) .
\end{aligned}
$$

The context of each variable can be seen in Fig. 4.3. One will notice that since the pseudo-spin eigenstates are a function of momentum, the tunnelling matrix element $\Gamma_{\mathrm{u} / \mathrm{l}}^{(\gamma)}$ is a function of $Q$ and $k_{\mathrm{F}}^{(m)}$. 


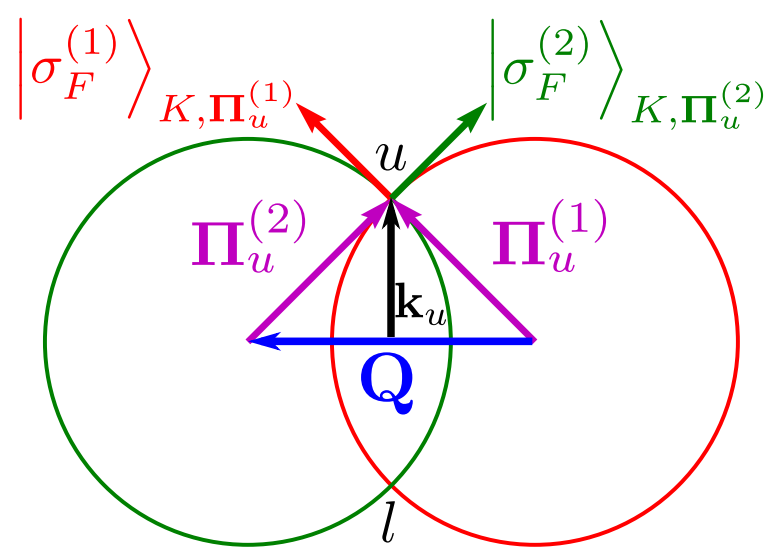

Figure 4.3: Here we see two Fermi-circles at the $\mathbf{K}$ valley for each layer, separated in momentum space by distance $Q$ (blue). The points which satisfy energy and momentum can be found at the intersection points ' $u$ ' and ' 1 '. $\mathbf{k}_{\mathrm{u} / \mathrm{l}}$ is the canonical momentum for each of these intersection points. The kinetic momentum at the ' $u$ ' intersection is represented via the purple arrows, and the direction of pseudo-spin eigenstates are shown using the green and red arrows. This diagram corresponds to two $n$-doped layers of SLG with equal electron densities.

\subsection{Pseudo-spin tunnelling matrix elements}

\subsubsection{Single layer graphene}

In this subsection, we investigate if System $1 n$ doped $\leftrightarrow$ System $2 n$ doped $(n \leftrightarrow$ $n$ ) tunnelling between two SLG layers is different from $p \leftrightarrow p$ doped tunnelling. Similarly, we see if $n \leftrightarrow p$ doped tunnelling is different from $n \leftrightarrow p$ doped tunnelling. In each case, we conclude there is no difference.

Using Eq. (2.7), the eigenstates at the $u$ intersection can be related to the other intersections (see Fig. 4.4). The angle of momentum at the $u$ intersection can be related to the angle at the $l$ intersection as a reflection along the $y$-axis. This provides the relation $\theta_{\boldsymbol{\Pi}_{\mathrm{u}}^{(m)}}=-\theta_{\boldsymbol{\Pi}_{1}^{(m)}}$, which we combine with Eq. 2.7) to show that

$$
\left|\sigma_{\mathrm{F}}^{(m)}\right\rangle_{\gamma, \boldsymbol{\Pi}_{1}^{(m)}}^{(\mathrm{slg})}=\sigma_{x}\left|\sigma_{\mathrm{F}}^{(m)}\right\rangle_{\gamma, \boldsymbol{\Pi}_{\mathrm{u}}^{(m)}}^{(\mathrm{slg})} .
$$

The pseudo-spin eigenstates in the $\mathbf{K}$ and $\mathbf{K}^{\prime}$ valleys are related through a reflection along the $x$-axis. For the $u$ intersection, this can be written as $\theta_{\boldsymbol{\Pi}_{\mathrm{u}}^{(m)}}^{\prime}=\pi-\theta_{\boldsymbol{\Pi}_{\mathrm{u}}^{(m)}}$ where $\theta_{\boldsymbol{\Pi}_{\mathbf{u}}^{(m)}}^{\prime}$ is the angle of pseudo-spin at the $u$ intersection for the $\mathbf{K}^{\prime}$ valley. Combining this relation with Eq. (2.7) shows that

$$
\left|\sigma_{\mathrm{F}}^{(m)}\right\rangle_{\mathbf{K}^{\prime}, \boldsymbol{\Pi}_{\mathrm{u} / 1}^{(m)}}^{(\mathrm{slg})}=\sigma_{y}\left|\sigma_{\mathrm{F}}^{(m)}\right\rangle_{\mathbf{K}, \boldsymbol{\Pi}_{\mathrm{u} / 1}^{(m)}}^{(\mathrm{slg})} .
$$

If one looks at the pseudo-spin eigenstates in Eq. (2.7), one will notice that the pseudo-spin eigenstates in SLG can be written using a rotation by the angle of 
momentum, $\mathcal{U}\left(\theta_{\boldsymbol{\Pi}^{(m)}}\right)=\exp \left(-i \sigma_{z} \theta_{\boldsymbol{\Pi}^{(m)}} / 2\right)$. For example, at the top intersection $u$, at the $\mathbf{K}$ valley

$$
|+\rangle_{\mathbf{K}, \boldsymbol{\Pi}_{\mathrm{u}}^{(m)}}^{(\mathrm{slg})}=\mathcal{U}\left(\theta_{\boldsymbol{\Pi}_{\mathrm{u}}^{(m)}}\right)|\rightarrow\rangle \quad \text { and } \quad|-\rangle_{\mathbf{K}, \boldsymbol{\Pi}_{\mathrm{u}}^{(m)}}^{(\mathrm{slg})}=\mathcal{U}\left(\theta_{\boldsymbol{\Pi}_{\mathrm{u}}^{(m)}}\right)|\leftarrow\rangle
$$

where $|\rightarrow\rangle=\frac{1}{\sqrt{2}}\left(\begin{array}{l}1 \\ 1\end{array}\right)$ and $|\leftarrow\rangle=\sigma_{z}|\rightarrow\rangle$.

Using the above relations for two $n$-doped layers of SLG ( which means $\sigma_{\mathrm{F}}^{(1)}=+$ and $\sigma_{\mathrm{F}}^{(2)}=+$ ), one can write the sum of tunnelling matrix contributions in Eq. (4.8) to find

$$
\begin{aligned}
& {\left.\left[\left|\Gamma_{\mathrm{u}}^{\mathbf{K}}\right|^{2}+\left|\Gamma_{1}^{\mathbf{K}}\right|^{2}+\left|\Gamma_{\mathrm{u}}^{\mathbf{K}^{\prime}}\right|^{2}+\left|\Gamma_{1}^{\mathbf{K}^{\prime}}\right|^{2}\right]\right|_{n \leftrightarrow n}=}\left|\left\langle\rightarrow\left|\mathcal{U}\left(\theta_{\boldsymbol{\Pi}_{\mathrm{u}}^{(2)}}\right)^{\dagger} \tau \mathcal{U}\left(\theta_{\boldsymbol{\Pi}_{\mathrm{u}}^{(1)}}\right)\right| \rightarrow\right\rangle\right|^{2}+\left|\left\langle\rightarrow\left|\mathcal{U}\left(\theta_{\boldsymbol{\Pi}_{\mathrm{u}}^{(2)}}\right)^{\dagger} \sigma_{x} \tau \sigma_{x} \mathcal{U}\left(\theta_{\boldsymbol{\Pi}_{\mathrm{u}}^{(1)}}\right)\right| \rightarrow\right\rangle\right|^{2} \\
&+\left|\left\langle\rightarrow\left|\mathcal{U}\left(\theta_{\boldsymbol{\Pi}_{\mathrm{u}}^{(2)}}\right)^{\dagger} \sigma_{y} \tau \sigma_{y} \mathcal{U}\left(\theta_{\boldsymbol{\Pi}_{\mathrm{u}}^{(1)}}\right)\right| \rightarrow\right\rangle\right|^{2}+\left|\left\langle\rightarrow\left|\mathcal{U}\left(\theta_{\boldsymbol{\Pi}_{\mathrm{u}}^{(2)}}\right)^{\dagger} \sigma_{z} \tau \sigma_{z} \mathcal{U}\left(\theta_{\boldsymbol{\Pi}_{\mathrm{u}}^{(1)}}\right)\right| \rightarrow\right\rangle\right|^{2} .
\end{aligned}
$$

One can then use the relation $|\rightarrow\rangle=\sigma_{z}|\leftarrow\rangle$ to show that this sum is the same for two $p$-doped layers of SLG (when $\sigma_{\mathrm{F}}^{(1)}=-$ and $\sigma_{\mathrm{F}}^{(2)}=-$ ),

$$
\left.\left[\left|\Gamma_{\mathrm{u}}^{\mathbf{K}}\right|^{2}+\left|\Gamma_{\mathrm{l}}^{\mathbf{K}}\right|^{2}+\left|\Gamma_{\mathrm{u}}^{\mathbf{K}^{\prime}}\right|^{2}+\left|\Gamma_{\mathrm{l}}^{\mathbf{K}^{\prime}}\right|^{2}\right]\right|_{n \leftrightarrow n}=\left.\left[\left|\Gamma_{\mathrm{u}}^{\mathbf{K}}\right|^{2}+\left|\Gamma_{\mathrm{l}}^{\mathbf{K}}\right|^{2}+\left|\Gamma_{\mathrm{u}}^{\mathbf{K}^{\prime}}\right|^{2}+\left|\Gamma_{1}^{\mathbf{K}^{\prime}}\right|^{2}\right]\right|_{p \leftrightarrow p}
$$

In conjunction with Eq. (4.8), this tells us that $G_{n \leftrightarrow n}^{(\mathrm{slg})}(0)=G_{p \leftrightarrow p}^{(\mathrm{slg})}(0)$. Following the same process, one can show that $G_{p \leftrightarrow n}^{(\mathrm{slg})}(0)=G_{n \leftrightarrow p}^{(\mathrm{slg})}(0)$ using

$$
\begin{aligned}
& {\left.\left[\left|\Gamma_{\mathrm{u}}^{\mathbf{K}}\right|^{2}+\left|\Gamma_{\mathrm{l}}^{\mathbf{K}}\right|^{2}+\left|\Gamma_{\mathrm{u}}^{\mathbf{K}^{\prime}}\right|^{2}+\left|\Gamma_{\mathrm{l}}^{\mathbf{K}^{\prime}}\right|^{2}\right]\right|_{p \leftrightarrow n}=} \\
& \quad\left|\left\langle\leftarrow\left|\mathcal{U}\left(\theta_{\boldsymbol{\Pi}_{\mathrm{u}}^{(2)}}\right)^{\dagger} \sigma_{z} \tau \sigma_{z} \mathcal{U}\left(\theta_{\boldsymbol{\Pi}_{\mathrm{u}}^{(1)}}\right)\right| \rightarrow\right\rangle\right|^{2}+\left|\left\langle\leftarrow\left|\mathcal{U}\left(\theta_{\boldsymbol{\Pi}_{\mathrm{u}}^{(2)}}\right)^{\dagger} \sigma_{y} \tau \sigma_{y} \mathcal{U}\left(\theta_{\boldsymbol{\Pi}_{\mathrm{u}}^{(1)}}\right)\right| \rightarrow\right\rangle\right|^{2} \\
& \quad+\left|\left\langle\leftarrow\left|\mathcal{U}\left(\theta_{\boldsymbol{\Pi}_{\mathrm{u}}^{(2)}}\right)^{\dagger} \sigma_{x} \tau \sigma_{x} \mathcal{U}\left(\theta_{\boldsymbol{\Pi}_{\mathrm{u}}^{(1)}}\right)\right| \rightarrow\right\rangle\right|^{2}+\left|\left\langle\leftarrow\left|\mathcal{U}\left(\theta_{\boldsymbol{\Pi}_{\mathrm{u}}^{(2)}}\right)^{\dagger} \tau \mathcal{U}\left(\theta_{\boldsymbol{\Pi}_{\mathrm{u}}^{(1)}}\right)\right| \rightarrow\right\rangle\right|^{2}
\end{aligned}
$$

One can then use $|\rightarrow\rangle=\sigma_{z}|\leftarrow\rangle$ to show

$$
\left.\left[\left|\Gamma_{\mathrm{u}}^{\mathbf{K}}\right|^{2}+\left|\Gamma_{\mathrm{l}}^{\mathbf{K}}\right|^{2}+\left|\Gamma_{\mathrm{u}}^{\mathbf{K}^{\prime}}\right|^{2}+\left|\Gamma_{\mathrm{l}}^{\mathbf{K}^{\prime}}\right|^{2}\right]\right|_{n \leftrightarrow p}=\left.\left[\left|\Gamma_{\mathrm{u}}^{\mathbf{K}}\right|^{2}+\left|\Gamma_{\mathrm{l}}^{\mathbf{K}}\right|^{2}+\left|\Gamma_{\mathrm{u}}^{\mathbf{K}^{\prime}}\right|^{2}+\left|\Gamma_{\mathrm{l}}^{\mathbf{K}^{\prime}}\right|^{2}\right]\right|_{p \leftrightarrow n}
$$

This shows that $n \leftrightarrow p$ tunnelling is the same as $p \leftrightarrow n$ tunnelling in the zero bias limit. 

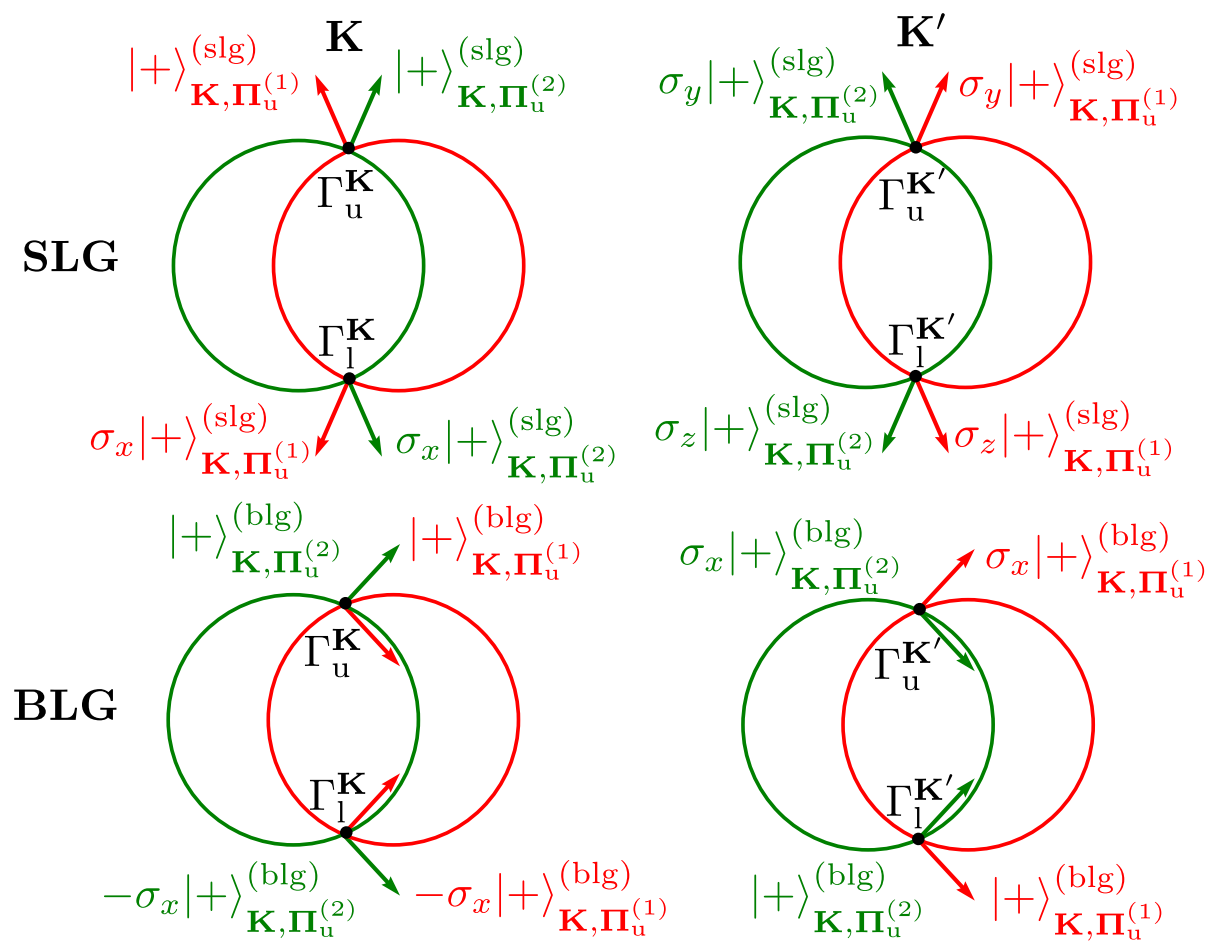

Figure 4.4: Intersecting Fermi circles for SLG and BLG. Each intersection point has been labelled with the corresponding tunnelling matrix element. The positive pseudospin eigenstate at the intersection points is represented by the arrows, with each eigenstate written in terms of $|+\rangle_{\mathbf{K}, \boldsymbol{\Pi}_{\mathrm{u}}^{(m)}}$. From this, one has a visual representation of the transformation relations shown in the text. 


\subsubsection{Bilayer graphene}

Now, we perform the same investigation as in the last subsection, but for tunnelling between two layers of BLG. We are interested in seeing if $n \leftrightarrow n$ doped tunnelling is different from $p \leftrightarrow p$ doped tunnelling. Similarly, we see if $n \leftrightarrow p$ doped tunnelling is different from $p \leftrightarrow n$ doped tunnelling. Unlike SLG, we find that swapping the doping of both layers of BLG between $n$ and $p$ changes the conductance.

The BLG pseudo-spin eigenstates in Eq. 2.14 can be used to relate the eigenstates of each intersection (see Fig. 4.4), using the same method as the previous subsection. The angles of momentum at the $u$ and $l$ intersections are related by $\theta_{\boldsymbol{\Pi}_{u}^{(m)}}=-\theta_{\Pi_{1}^{(m)}}$. Using Eq. 2.14 we find the relation

$$
\left|\sigma_{\mathrm{F}}^{(m)}\right\rangle_{\gamma, \boldsymbol{\Pi}_{1}^{(m)}}^{(\mathrm{blg})}=-\sigma_{x}\left|\sigma_{\mathrm{F}}^{(m)}\right\rangle_{\gamma, \boldsymbol{\Pi}_{\mathrm{u}}^{(m)}}^{(\mathrm{blg})} .
$$

However, the $\mathbf{K}$ and $\mathbf{K}^{\prime}$ valley pseudo-spin eigenstates are related through $\theta_{\mathbf{\Pi}_{\mathrm{u}}^{(m)}}^{\prime}=$ $\pi-\theta_{\Pi_{u}^{(m)}}$. Using Eq. 2.14 we find the relation

$$
\left|\sigma_{\mathrm{F}}^{(m)}\right\rangle_{\mathbf{K}^{\prime}, \boldsymbol{\Pi}_{\mathbf{u} / 1}^{(m)}}^{(\mathrm{blg})}=\sigma_{x}\left|\sigma_{\mathrm{F}}^{(m)}\right\rangle_{\mathbf{K}, \boldsymbol{\Pi}_{\mathbf{u} / 1}^{(m)}}^{(\mathrm{blg})} .
$$

Similar to SLG, if one looks at the pseudo-spin eigenstates in Eq. 2.14, one will notice that the pseudo-spin eigenstates in BLG can be obtained using a rotation by the angle of momentum, $\mathcal{U}\left(-2 \theta_{\left.\boldsymbol{\Pi}^{(m)}\right)}\right.$. For example, at the $u$ intersection of the $\mathbf{K}$ valley

$$
|+\rangle_{\mathbf{K}, \boldsymbol{\Pi}_{\mathrm{u}}^{(m)}}^{(\mathrm{blg})}=\mathcal{U}\left(-2 \theta_{\boldsymbol{\Pi}_{\mathrm{u}}^{(m)}}\right)|\leftarrow\rangle \quad \text { and } \quad|-\rangle_{\mathbf{K}, \boldsymbol{\Pi}_{\mathrm{u}}^{(m)}}^{(\mathrm{blg})}=\sigma_{z}|+\rangle_{\mathbf{K}, \boldsymbol{\Pi}_{\mathrm{u}}^{(m)}}^{(\mathrm{blg})} .
$$

Using the relations above, one can write the sum of tunnelling matrix contributions in Eq. (4.8) for $n \leftrightarrow n$ tunnelling as

$$
\begin{aligned}
& {\left.\left[\left|\Gamma_{\mathrm{u}}^{\mathbf{K}}\right|^{2}+\left|\Gamma_{1}^{\mathbf{K}}\right|^{2}+\left|\Gamma_{\mathrm{u}}^{\mathbf{K}^{\prime}}\right|^{2}+\left|\Gamma_{1}^{\mathbf{K}^{\prime}}\right|^{2}\right]\right|_{n \leftrightarrow n}=} \\
& 2\left|\left\langle\leftarrow\left|\mathcal{U}\left(-2 \theta_{\boldsymbol{\Pi}_{\mathrm{u}}^{(2)}}\right)^{\dagger} \tau \mathcal{U}\left(-2 \theta_{\boldsymbol{\Pi}_{\mathrm{u}}^{(1)}}\right)\right| \leftarrow\right\rangle\right|^{2}+2\left|\left\langle\leftarrow\left|\mathcal{U}\left(-2 \theta_{\boldsymbol{\Pi}_{\mathrm{u}}^{(2)}}\right)^{\dagger} \sigma_{x} \tau \sigma_{x} \mathcal{U}\left(-2 \theta_{\boldsymbol{\Pi}_{\mathrm{u}}^{(1)}}\right)\right| \leftarrow\right\rangle\right|^{2}
\end{aligned}
$$

where we use $\sigma_{\mathrm{F}}^{(1)}=+$ and $\sigma_{\mathrm{F}}^{(2)}=+$ for each system. Using the relation $|\rightarrow\rangle=\sigma_{z}|\leftarrow\rangle$, one will find that the sum is different for $p \leftrightarrow p$ tunnelling

$$
\begin{gathered}
{\left.\left[\left|\Gamma_{\mathrm{u}}^{\mathbf{K}}\right|^{2}+\left|\Gamma_{1}^{\mathbf{K}}\right|^{2}+\left|\Gamma_{\mathrm{u}}^{\mathbf{K}^{\prime}}\right|^{2}+\left|\Gamma_{1}^{\mathbf{K}^{\prime}}\right|^{2}\right]\right|_{p \leftrightarrow p}=} \\
2\left|\left\langle\leftarrow\left|\mathcal{U}\left(-2 \theta_{\boldsymbol{\Pi}_{\mathrm{u}}^{(2)}}\right)^{\dagger} \sigma_{z} \tau \sigma_{z} \mathcal{U}\left(-2 \theta_{\boldsymbol{\Pi}_{\mathrm{u}}^{(1)}}\right)\right| \leftarrow\right\rangle\right|^{2}+2\left|\left\langle\leftarrow\left|\mathcal{U}\left(-2 \theta_{\boldsymbol{\Pi}_{\mathrm{u}}^{(2)}}\right)^{\dagger} \sigma_{y} \tau \sigma_{y} \mathcal{U}\left(-2 \theta_{\boldsymbol{\Pi}_{\mathrm{u}}^{(1)}}\right)\right| \leftarrow\right\rangle\right|^{2}
\end{gathered}
$$

where $\sigma_{\mathrm{F}}^{(1)}=-$ and $\sigma_{\mathrm{F}}^{(2)}=-$. From the above, we find

$$
\left.\left[\left|\Gamma_{\mathrm{u}}^{\mathbf{K}}\right|^{2}+\left|\Gamma_{1}^{\mathbf{K}}\right|^{2}+\left|\Gamma_{\mathrm{u}}^{\mathbf{K}^{\prime}}\right|^{2}+\left|\Gamma_{1}^{\mathbf{K}^{\prime}}\right|^{2}\right]\right|_{n \leftrightarrow n} \neq\left.\left[\left|\Gamma_{\mathrm{u}}^{\mathbf{K}}\right|^{2}+\left|\Gamma_{1}^{\mathbf{K}}\right|^{2}+\left|\Gamma_{\mathrm{u}}^{\mathbf{K}^{\prime}}\right|^{2}+\left|\Gamma_{1}^{\mathbf{K}^{\prime}}\right|^{2}\right]\right|_{p \leftrightarrow p}
$$


which means that $G_{n \leftrightarrow n}^{(\mathrm{blg})}(0) \neq G_{p \leftrightarrow p}^{(\mathrm{blg})}(0)$.

Following the same process, one can show that $G_{p \leftrightarrow n}^{(\mathrm{blg})}(0) \neq G_{n \leftrightarrow p}^{(\mathrm{blg})}(0)$. One can write the sum of tunnelling matrix contributions in Eq. (4.8) for $n \rightarrow p$ tunnelling as

$$
\begin{aligned}
& {\left.\left[\left|\Gamma_{\mathrm{u}}^{\mathbf{K}}\right|^{2}+\left|\Gamma_{1}^{\mathbf{K}}\right|^{2}+\left|\Gamma_{\mathrm{u}}^{\mathbf{K}^{\prime}}\right|^{2}+\left|\Gamma_{1}^{\mathbf{K}^{\prime}}\right|^{2}\right]\right|_{n \leftrightarrow p}=} \\
& 2\left|\left\langle\leftarrow\left|\mathcal{U}\left(-2 \theta_{\boldsymbol{\Pi}_{\mathrm{u}}^{(2)}}\right)^{\dagger} \tau \mathcal{U}\left(-2 \theta_{\boldsymbol{\Pi}_{\mathrm{u}}^{(1)}}\right)\right| \rightarrow\right\rangle\right|^{2}+2\left|\left\langle\leftarrow\left|\mathcal{U}\left(-2 \theta_{\boldsymbol{\Pi}_{\mathrm{u}}^{(2)}}\right)^{\dagger} \sigma_{x} \tau \sigma_{x} \mathcal{U}\left(-2 \theta_{\boldsymbol{\Pi}_{\mathrm{u}}^{(1)}}\right)\right| \rightarrow\right\rangle\right|^{2}
\end{aligned}
$$

where we use $\sigma_{\mathrm{F}}^{(1)}=+$ and $\sigma_{\mathrm{F}}^{(2)}=-$ for each system. Using the relation $|\rightarrow\rangle=\sigma_{z}|\leftarrow\rangle$, one will find that the sum is different for $p \rightarrow p$ tunnelling

$$
\begin{gathered}
{\left.\left[\left|\Gamma_{\mathrm{u}}^{\mathbf{K}}\right|^{2}+\left|\Gamma_{l}^{\mathbf{K}}\right|^{2}+\left|\Gamma_{\mathrm{u}}^{\mathbf{K}^{\prime}}\right|^{2}+\left|\Gamma_{l}^{\mathbf{K}^{\prime}}\right|^{2}\right]\right|_{p \leftrightarrow n}=} \\
2\left|\left\langle\leftarrow\left|\mathcal{U}\left(-2 \theta_{\boldsymbol{\Pi}_{\mathrm{u}}^{(2)}}\right)^{\dagger} \sigma_{z} \tau \sigma_{z} \mathcal{U}\left(-2 \theta_{\boldsymbol{\Pi}_{\mathrm{u}}^{(1)}}\right)\right| \rightarrow\right\rangle\right|^{2}+2\left|\left\langle\leftarrow\left|\mathcal{U}\left(-2 \theta_{\boldsymbol{\Pi}_{\mathrm{u}}^{(2)}}\right)^{\dagger} \sigma_{y} \tau \sigma_{y} \mathcal{U}\left(-2 \theta_{\boldsymbol{\Pi}_{\mathrm{u}}^{(1)}}\right)\right| \rightarrow\right\rangle\right|^{2}
\end{gathered}
$$

where $\sigma_{\mathrm{F}}^{(1)}=-$ and $\sigma_{\mathrm{F}}^{(2)}=+$. From the above, we find

$$
\left.\left[\left|\Gamma_{\mathrm{u}}^{\mathbf{K}}\right|^{2}+\left|\Gamma_{\mathrm{l}}^{\mathbf{K}}\right|^{2}+\left|\Gamma_{\mathrm{u}}^{\mathbf{K}^{\prime}}\right|^{2}+\left|\Gamma_{\mathrm{l}}^{\mathbf{K}^{\prime}}\right|^{2}\right]\right|_{n \leftrightarrow p} \neq\left.\left[\left|\Gamma_{\mathrm{u}}^{\mathbf{K}}\right|^{2}+\left|\Gamma_{\mathrm{l}}^{\mathbf{K}}\right|^{2}+\left|\Gamma_{\mathrm{u}}^{\mathbf{K}^{\prime}}\right|^{2}+\left|\Gamma_{\mathrm{l}}^{\mathbf{K}^{\prime}}\right|^{2}\right]\right|_{p \leftrightarrow n}
$$

which means that $G_{n \leftrightarrow p}^{(\mathrm{blg})}(0) \neq G_{p \leftrightarrow n}^{(\mathrm{blg})}(0)$ at zero bias. However, there are cases when $G_{n \leftrightarrow p}^{(\mathrm{blg})}(0)=G_{p \leftrightarrow n}^{(\mathrm{blg})}(0)$, i.e. when pseudo-spin is conserved.

We have shown that $G_{n \leftrightarrow p}^{(\mathrm{blg})}(0) \neq G_{p \leftrightarrow n}^{(\mathrm{blg})}(0)$ when there is zero bias between system 1 and system 2 . This suggests that system 1 and system 2 can be distinguished in the zero bias limit. However, it may be that $G_{n \rightarrow p}^{(\mathrm{blg})}(0)=G_{p \rightarrow n}^{(\mathrm{blg})}(0)$ is required for physicality, this would simplify the possible the pseudo-spin couplings between the two layers of BLG.

\subsubsection{Tunnelling matrix parametrisation}

The most convenient way to evaluate the tunnelling matrix element is to represent the tunnelling matrix in terms of the basis of Pauli matrices

$$
\tau=\frac{1}{\sqrt{2}}\left[\tau_{0} \sigma_{0}+\tau_{x} \sigma_{x}+\tau_{y} \sigma_{y}+\tau_{z} \sigma_{z}\right]
$$

$\tau_{\eta}$ are complex numbers that encode pseudo-spin flips about the $\eta^{\text {th }}$ axis. For instance, we find

$$
\Gamma_{\mathrm{u} / 1}^{(\gamma)}=\sum_{\eta=0, x, y, z} \frac{1}{\sqrt{2}} \tau_{\eta, \mathbf{k}_{\mathrm{u} / 1}} \gamma, \boldsymbol{\Pi}_{\mathrm{u} / 1}^{(1)}\left\langle\sigma_{\mathrm{F}}^{(1)}\left|\sigma_{\eta}\right| \sigma_{\mathrm{F}}^{(2)}\right\rangle_{\gamma, \boldsymbol{\Pi}_{\mathrm{u} / 1}^{(2)}}
$$



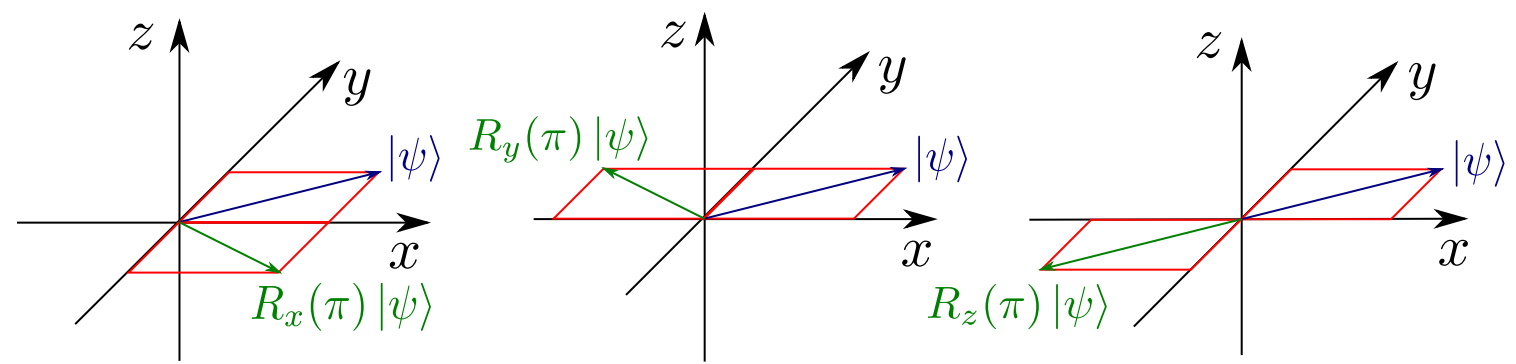

Figure 4.5: The three different types of pseudo-spin flips for a pseudo-spin state in the $x y$ plane. The pseudo-spin before interaction with the barrier, $|\psi\rangle$, is shown in blue. The pseudo-spin after the flip about the $x, y, z$ axis, $R_{x, y, z}(\pi)|\psi\rangle$, is shown in green. Each of these processes are encoded in the pseudo-spin tunnelling matrix, through the parameters $\tau_{x}, \tau_{y}, \tau_{z}$.

From the relations between the Pauli matrices and the pseudo-spin rotation operator $R_{\eta}(\theta)=\exp \left[-i \frac{\theta}{2} \sigma_{\eta}\right]$

$$
\sigma_{x}=i R_{x}(\pi), \quad \sigma_{y}=i R_{y}(\pi), \quad \sigma_{z}=i R_{z}(\pi),
$$

we can see how the Pauli matrices represent pseudo-spin flips about a given axis due to the tunnelling process [56]. $\tau_{\eta}$ would be a phase and amplitude attached to such a process. An example of each pseduo-spin flip is shown in Fig. 4.5.

From the kinetic momentum dependence of the pseudo-spin eigenstates, one can calculate

$$
\gamma, \Pi_{\mathbf{u} / 1}^{(1)}\left\langle\sigma_{\mathrm{F}}^{(1)}\left|\sigma_{\eta}\right| \sigma_{\mathrm{F}}^{(2)}\right\rangle_{\gamma, \boldsymbol{\Pi}_{\mathrm{u} / 1}^{(2)}}
$$

as a function of $Q$, and $k_{\mathrm{F}}^{(m)}$. For example, one will find the straightforward calculation for SLG

$$
\underset{\gamma, \boldsymbol{\Pi}_{\mathrm{u} / 1}^{(1)}\langle(\mathrm{s}}{(\mathrm{s})}\left|\sigma_{\mathrm{F}}^{(1)}\right| \sigma_{0}\left|\sigma_{\mathrm{F}}^{(2)}\right\rangle_{\gamma, \boldsymbol{\Pi}_{\mathrm{u} / 1}^{(2)}}^{(\mathrm{slg})}=\cos \left(\frac{\theta_{\boldsymbol{\Pi}_{\mathrm{u} / 1}^{(1)}-\theta_{\boldsymbol{\Pi}_{\mathrm{u} / 1}^{(2)}}}}{2}\right) .
$$

One can then use trigonometric identities to obtain the pseudo-spin overlap as a function of $\cos \theta_{\boldsymbol{\Pi}_{\mathrm{u} / 1}^{(m)}}$ and $\sin \theta_{\boldsymbol{\Pi}_{\mathrm{u} / 1}^{(m)}}$, the momentum direction at the intersections of the Fermi circles. Once in this form, one can use Eqs. 4.10 to obtain algebraic formulae at the ' $u$ ' intersection,

$$
\begin{array}{r}
\cos \theta_{\Pi_{\mathrm{u}}^{(1)}}=\frac{k_{\mathrm{F}}^{(2) 2}-k_{\mathrm{F}}^{(1) 2}-Q^{2}}{2 Q k_{\mathrm{F}}^{(1)}}, \quad \cos \theta_{\Pi_{\mathrm{u}}^{(2)}}=\frac{k_{\mathrm{F}}^{(2) 2}-k_{\mathrm{F}}^{(1) 2}+Q^{2}}{2 Q k_{\mathrm{F}}^{(2)}}, \\
\sin \theta_{\Pi_{\mathrm{u}}^{(1)}}=\frac{\sqrt{\left[\left(k_{\mathrm{F}}^{(1)}+k_{\mathrm{F}}^{(2)}\right)^{2}-Q^{2}\right]\left[Q^{2}-\left(k_{\mathrm{F}}^{(1)}-k_{\mathrm{F}}^{(2)}\right)^{2}\right]}}{2 Q k_{\mathrm{F}}^{(1)}}, \\
\sin \theta_{\Pi_{\mathrm{u}}^{(2)}}=\frac{\sqrt{\left[\left(k_{\mathrm{F}}^{(1)}+k_{\mathrm{F}}^{(2)}\right)^{2}-Q^{2}\right]\left[Q^{2}-\left(k_{\mathrm{F}}^{(1)}-k_{\mathrm{F}}^{(2)}\right)^{2}\right]}}{2 Q k_{\mathrm{F}}^{(2)}} .
\end{array}
$$


For the 'l' intersection,

$$
\cos \theta_{\boldsymbol{\Pi}_{1}^{(m)}}=\cos \theta_{\boldsymbol{\Pi}_{\mathrm{u}}^{(m)}}, \quad \sin \theta_{\boldsymbol{\Pi}_{1}^{(m)}}=-\sin \theta_{\boldsymbol{\Pi}_{\mathrm{u}}^{(m)}} .
$$

See Fig. 4.3 to see the ' $u$ ' and 'l' intersections of the intersecting Fermi circles.

\subsection{Comparative study of chiral magneto-tunnelling transport}

In this section, we investigate the derived linear magneto-tunnelling conductance formula Eq. (4.8), using different chiral materials and for different dopants in the presence of an in-plane magnetic field. We present and compare the linear magnetotunnelling conductance for graphene, bilayer graphene, and $\mathrm{MoS}_{2}$.

From now on, we assume that $\tau_{\mathbf{k}} \equiv \tau$ has no momentum dependence. This has been shown to be a reasonable assumption in Ref. [23. We will also choose the notation

$$
\tau=\frac{1}{\sqrt{2}}\left[\tau_{0} \sigma_{0}+\tau_{\perp} \sigma_{\perp}+\tau_{\|} \sigma_{\|}+\tau_{z} \sigma_{z}\right]
$$

where $\perp$ and $\|$ are the in-plane axes perpendicular and parallel to the in-plane magnetic field (for example one could choose $x=\perp$ and $y=\|$ ).

\subsubsection{Single layer graphene}

To calculate the linear magneto-tunnelling conductance for SLG, one uses

$$
\varepsilon_{\gamma, \mathbf{k}, \sigma}^{(\operatorname{slg})}=\sigma v\|\mathbf{\Pi}(\mathbf{k}, \mathbf{B})\|
$$

and the pseudo-spin eigenstates

$$
|\sigma\rangle_{\mathbf{K}, \boldsymbol{\Pi}}^{(\mathrm{slg})}=\frac{1}{\sqrt{2}}\left(\begin{array}{c}
\mathrm{e}^{-i \theta_{\boldsymbol{\Pi}} / 2} \\
\sigma \mathrm{e}^{i \theta_{\boldsymbol{\Pi}} / 2}
\end{array}\right) \quad|\sigma\rangle_{\mathbf{K}^{\prime}, \boldsymbol{\Pi}}^{(\mathrm{slg})}=\frac{1}{\sqrt{2}}\left(\begin{array}{c}
\mathrm{e}^{-i\left(\pi-\theta_{\boldsymbol{\Pi}}\right) / 2} \\
\sigma \mathrm{e}^{i\left(\pi-\theta_{\boldsymbol{\Pi}}\right) / 2}
\end{array}\right) .
$$

One can now use Eq. 4.8 to calculate the magneto-tunnelling conductance.

The linear magneto-tunnelling conductance between two $n$ doped SLG systems is

$$
\begin{array}{r}
\frac{G_{n \leftrightarrow n}^{(\mathrm{slg})}(0)}{G_{0}}=\frac{\Theta(Q-\Delta) \Theta\left(2 \bar{k}_{\mathrm{F}}-Q\right)}{\operatorname{Tr}\left[\tau^{\dagger} \tau\right]}\left\{\left[\left|\tau_{0}\right|^{2}+\left|\tau_{\perp}\right|^{2} \frac{\Delta^{2}}{Q^{2}}\right] \sqrt{\frac{4 \bar{k}_{\mathrm{F}}^{2}-Q^{2}}{Q^{2}-\Delta^{2}}}\right. \\
\left.+\left[\left|\tau_{z}\right|^{2}+\left|\tau_{\|}\right|^{2} \frac{4 \bar{k}_{\mathrm{F}}^{2}}{Q^{2}}\right] \sqrt{\frac{Q^{2}-\Delta^{2}}{4 \bar{k}_{\mathrm{F}}^{2}-Q^{2}}}\right\} .
\end{array}
$$

We find that pseudo-spin flips, $\tau_{\perp}, \tau_{\|}, \tau_{z}$ in the above equation, can activate the resonances seen at $Q=2 \bar{k}_{\mathrm{F}}$ and $Q=\Delta$. 
For tunnelling between $n$ and $p$ doped systems, the magneto-tunnelling conductance is

$$
\begin{aligned}
\frac{G_{n \rightarrow p}^{(\mathrm{slg})}(0)}{G_{0}}=\frac{\Theta(Q-\Delta) \Theta\left(2 \bar{k}_{F}-Q\right)}{\operatorname{Tr}\left[\tau^{\dagger} \tau\right]}\left\{\left[\left|\tau_{0}\right|^{2}+\left|\tau_{\perp}\right|^{2} \frac{4 \bar{k}_{F}^{2}}{Q^{2}}\right] \sqrt{\frac{Q^{2}-\Delta^{2}}{4 \bar{k}_{F}^{2}-Q^{2}}}\right. & \\
+ & {\left.\left[\left|\tau_{z}\right|^{2}+\left|\tau_{\|}\right|^{2} \frac{\Delta^{2}}{Q^{2}}\right] \sqrt{\frac{4 \bar{k}_{F}^{2}-Q^{2}}{Q^{2}-\Delta^{2}}}\right\} . }
\end{aligned}
$$

One will notice that $G_{n \rightarrow n}^{(\mathrm{slg})}(0)$ has a similar form to $G_{n \rightarrow p}^{(\mathrm{slg})}(0)$, where the resonances are the same but depend on different parameters. This is because the conduction and valence band eignstates are related by a pseudo-spin flip about the $z$-axis, $\sigma_{z}|+\rangle=$ $|-\rangle$. This effectively swaps the roles between $\tau_{0}$ and $\tau_{z}$, as well as $\tau_{x}$ and $\tau_{y}$.

For equal densities $(\Delta=0)$ and pseudo-spin conservation in the tunnelling process $\left(\tau=\frac{1}{\sqrt{2}} \tau_{0} \sigma_{0}\right)$, one can generally show that

$$
\frac{G^{(\mathrm{slg})}(0)}{G_{0}^{(\mathrm{slg})}}=\left|{ }_{\mathbf{K}, \boldsymbol{\Pi}_{\mathrm{u}}^{(1)}}^{(\mathrm{slg})}\left\langle\sigma_{\mathrm{F}}^{(1)} \mid \sigma_{\mathrm{F}}^{(2)}\right\rangle_{\mathbf{K}, \boldsymbol{\Pi}_{\mathrm{u}}^{(2)}}^{(\mathrm{slg})}\right|^{2} \frac{G^{(2 \mathrm{deg})}(0)}{G_{0}^{(2 \mathrm{deg})}},
$$

which means the characteristics only differ from the 2DEG due to the pseudo-spin overlap at the $u$ intersection. Since the pseudo-spin overlap is bounded between 0 and 1 , the chiral eigenstates can only dampen the conductance. For two $n$ doped layers, one will find

$$
\frac{G_{n \leftrightarrow n}^{(\mathrm{slg})}(0)}{G_{0}}=\frac{\sqrt{4 \bar{k}_{\mathrm{F}}^{2}-Q^{2}}}{Q} \Theta\left(2 \bar{k}_{\mathrm{F}}-Q\right) .
$$

The pseudo-spins are parallel when the Fermi circles overlap $(Q=0)$, and continuously become orthogonal as the Fermi circles kiss. In particular, the pseudo-spin overlap removes the resonance at the kissing point $\left(Q=2 \bar{k}_{\mathrm{F}}\right)$. See Fig. 4.6 for a plot of Eq. 4.41).

Now one can investigate

$$
\frac{G_{n \leftrightarrow p}^{(\mathrm{slg})}(0)}{G_{0}}=\frac{Q}{\sqrt{4 \bar{k}_{\mathrm{F}}^{2}-Q^{2}}} \Theta\left(2 \bar{k}_{\mathrm{F}}-Q\right) .
$$

For two $n$ and $p$ doped layers, the pseudo-spins are orthogonal when the Fermi circles overlap $(Q=0)$, but become parallel at the kissing point $\left(Q=2 \bar{k}_{\mathrm{F}}\right)$. This removes the resonance at $Q=0$, while preserving the resonance at $Q=2 \bar{k}_{\mathrm{F}}$. Essentially, the reverse situation of two $n$ doped layers, because of the pseudo-spin relation $\sigma_{z}|+\rangle=|-\rangle$ between the conduction and valence band. See Fig. 4.7.

\subsubsection{Bilayer graphene}

In BLG, the energy is similar to a $2 \mathrm{DEG}$

$$
\varepsilon_{\gamma, \mathbf{k}, \sigma}^{(\mathrm{blg})}=\sigma \frac{1}{2 M}\|\mathbf{\Pi}(\mathbf{k}, \mathbf{B})\|^{2}
$$


and the pseudo-spin eigenstates are

$$
|\sigma\rangle_{\mathbf{K}, \boldsymbol{\Pi}}=\frac{1}{\sqrt{2}}\left(\begin{array}{c}
\mathrm{e}^{i \theta_{\boldsymbol{\Pi}}} \\
-\sigma \mathrm{e}^{-i \theta_{\boldsymbol{\Pi}}}
\end{array}\right) \quad|\sigma\rangle_{\mathbf{K}^{\prime}, \boldsymbol{\Pi}}=\frac{1}{\sqrt{2}}\left(\begin{array}{c}
\mathrm{e}^{-i \theta_{\boldsymbol{\Pi}}} \\
-\sigma \mathrm{e}^{i \theta_{\boldsymbol{\Pi}}}
\end{array}\right) .
$$

The linear magneto-tunnelling conductance between two $n$ doped layers is

$$
\begin{aligned}
& \frac{G_{n \leftrightarrow n}^{(\mathrm{blg})}(0)}{G_{0}}=\frac{\Theta(Q-\Delta) \Theta\left(2 \bar{k}_{\mathrm{F}}-Q\right)}{\operatorname{Tr}\left[\tau^{\dagger} \tau\right]} \times \\
& \quad\left\{\frac{\left|\tau_{0}\left(4 \bar{k}_{\mathrm{F}}^{2}+\Delta^{2}-2 Q^{2}\right) Q^{2}-\tau_{\perp}\left[8 \bar{k}_{\mathrm{F}}^{2} \Delta^{2}-\left(4 \bar{k}_{\mathrm{F}}^{2}+\Delta^{2}\right) Q^{2}\right]\right|^{2}}{Q^{4}\left(4 \bar{k}_{\mathrm{F}}^{2}-\Delta^{2}\right) \sqrt{\left(4 \bar{k}_{\mathrm{F}}^{2}-Q^{2}\right)\left(Q^{2}-\Delta^{2}\right)}}\right. \\
& \left.+\left|\tau_{\|} 8 \bar{k}_{\mathrm{F}}^{2} \Delta^{2}-i \tau_{z} 2 Q^{2}\right|^{2} \frac{\sqrt{\left(4 \bar{k}_{\mathrm{F}}^{2}-Q^{2}\right)\left(Q^{2}-\Delta^{2}\right)}}{Q^{4}\left(4 \bar{k}_{\mathrm{F}}^{2}-\Delta^{2}\right)}\right\}
\end{aligned}
$$

For tunnelling between an $n$ doped and $p$ doped system, the linear magneto-tunnelling conductance is

$$
\begin{aligned}
\frac{G_{n \rightarrow p}^{(\mathrm{blg})}(0)}{G_{0}}= & \frac{\Theta(Q-\Delta) \Theta\left(2 \bar{k}_{\mathrm{F}}-Q\right)}{\operatorname{Tr}\left[\tau^{\dagger} \tau\right]}\left\{\left|\tau_{0} 2 Q^{2}+\tau_{\perp} 8 \bar{k}_{\mathrm{F}}^{2} \Delta^{2}\right|^{2} \frac{\sqrt{\left(4 \bar{k}_{\mathrm{F}}^{2}-Q^{2}\right)\left(Q^{2}-\Delta^{2}\right)}}{Q^{4}\left(4 \bar{k}_{\mathrm{F}}^{2}-\Delta^{2}\right)}\right. \\
& \left.+\frac{\left|\tau_{\|}\left[8 \bar{k}_{\mathrm{F}}^{2} \Delta^{2}-\left(4 \bar{k}_{\mathrm{F}}^{2}+\Delta^{2}\right) Q^{2}\right]+i \tau_{z}\left(4 \bar{k}_{\mathrm{F}}^{2}+\Delta^{2}-2 Q^{2}\right) Q^{2}\right|^{2}}{Q^{4}\left(4 \bar{k}_{\mathrm{F}}^{2}-\Delta^{2}\right) \sqrt{\left(4 \bar{k}_{\mathrm{F}}^{2}-Q^{2}\right)\left(Q^{2}-\Delta^{2}\right)}}\right\}
\end{aligned}
$$

When pseudo-spin is conserved in tunnelling and the densities in each layer are equal, we find the familiar relation

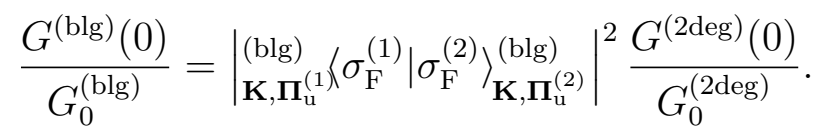

For two $n$ doped layers, one finds

$$
\frac{G_{n \leftrightarrow n}^{(\mathrm{blg})}(0)}{G_{0}}=\frac{\left(2 \bar{k}_{F}^{2}-Q^{2}\right)^{2}}{\bar{k}_{\mathrm{F}}^{2} Q \sqrt{4 \bar{k}_{F}^{2}-Q^{2}}} \Theta\left(2 \bar{k}_{\mathrm{F}}-Q\right) .
$$

In this case, the divergences when the Fermi circles overlap $(Q=0)$ and kiss $\left(Q=2 \bar{k}_{F}\right)$ are still present, because the pseudo-spins are parallel. However, when $Q=\sqrt{2} \bar{k}_{F}$, the pseudo-spins are orthogonal. This causes the magneto-tunnelling conductance to vanish at $Q=\sqrt{2} \bar{k}_{F}$, and dampen near this location. See Fig. 4.6 for a plot of Eq. 4.49. For tunnelling between $n$ and $p$ doped layers,

$$
\frac{G_{n \leftrightarrow p}^{(\mathrm{blg})}(0)}{G_{0}}=\frac{Q \sqrt{4 \bar{k}_{\mathrm{F}}^{2}-Q^{2}}}{\bar{k}_{\mathrm{F}}^{2}} .
$$

Now one finds, the magneto-tunnelling conductance vanishes at $Q=0$ and $Q=2 \bar{k}_{F}$ due to orthogoanl pseudo-spins, removing both resonances. The maximum of the magneto-tunnelling conductance is when $Q=\sqrt{2} \bar{k}_{F}$, where the pseudo-spins are parallel at the Fermi circle intersections. See Fig. 4.7. 


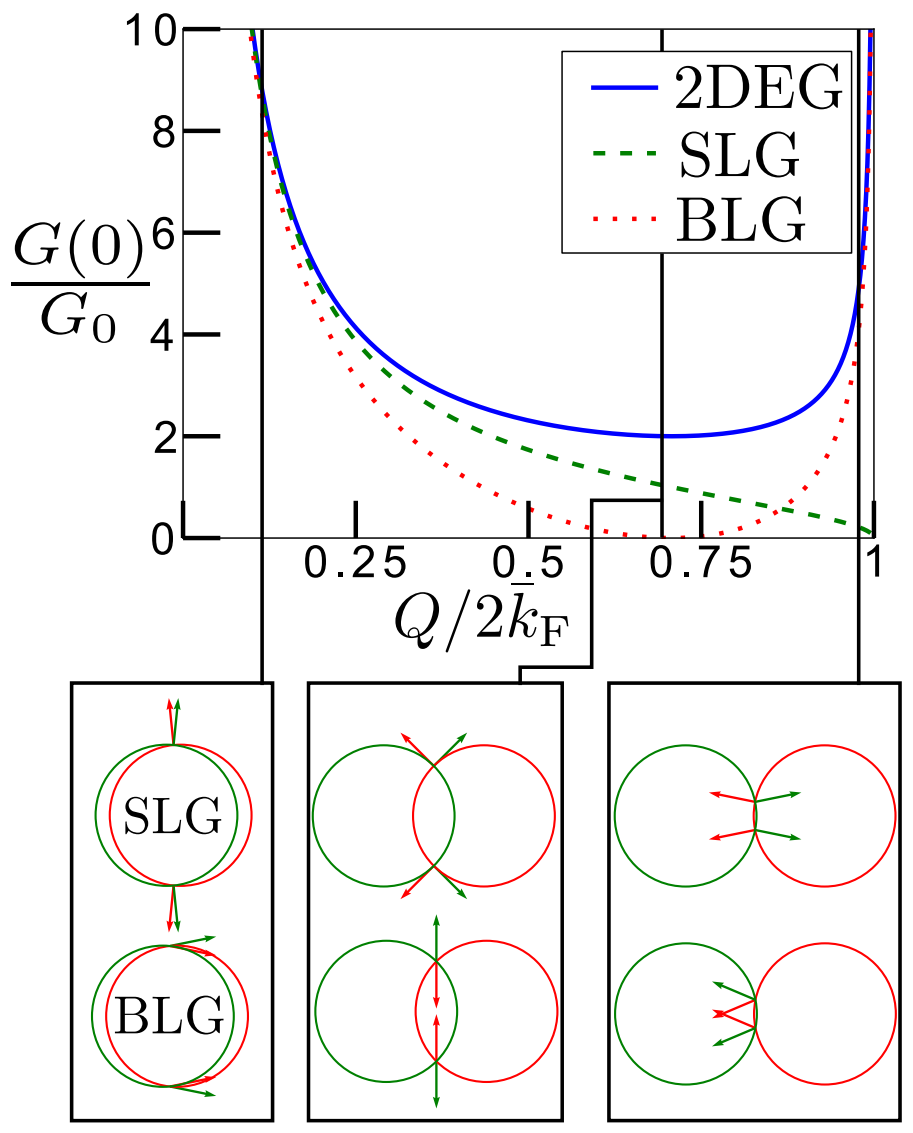

Figure 4.6: Here we compare the linear magneto-tunnelling conductance as a function of $Q / 2 \bar{k}_{\mathrm{F}}$ between two $n$ doped layers of 2DEG (blue), SLG (green), and BLG (red), when the densities in the layers are equal and pseudo-spin is conserved in tunnelling. Below the plot are diagrams of the intersecting Fermi circles with the direction of pseudo-spin for SLG and BLG, for values of $Q=0.2 \bar{k}_{\mathrm{F}}, \sqrt{2} \bar{k}_{\mathrm{F}}, 1.96 \bar{k}_{\mathrm{F}}$, as indicated by the black lines on the plot. The 2DEG shows a resonance when the Fermi-circles kiss and overlap. For SLG, the pseudo-spins at the intersections become orthogonal as $Q \rightarrow 2 \bar{k}_{\mathrm{F}}$, which dampens the conductance and removes the divergence at the kissing point $Q=2 \bar{k}_{\mathrm{F}}$. For BLG, the pseudo-spins at the intersections are parallel when $Q=0,2 \bar{k}_{\mathrm{F}}$, but become orthogonal as $Q \rightarrow \sqrt{2} \bar{k}_{\mathrm{F}}$, which dampens the conductance close to this point. 


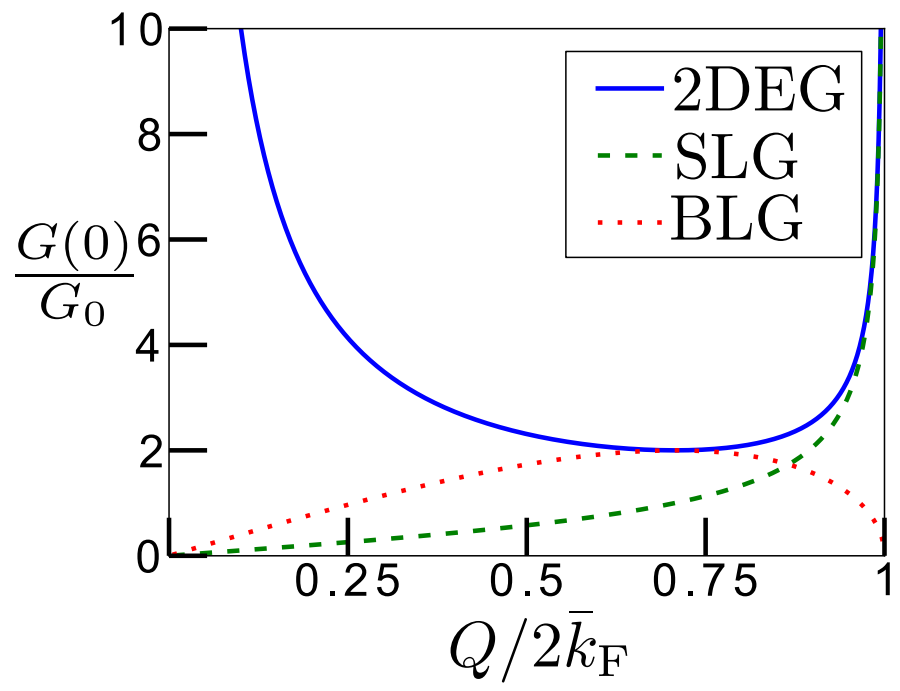

Figure 4.7: The linear magneto-tunnelling conductance as a function of $Q / 2 \bar{k}_{\mathrm{F}}$ between two $n \leftrightarrow p$ doped layers of 2DEG (blue), SLG (green), and BLG (red), when the densities in the layers are equal and pseudo-spin is conserved in tunnelling. The 2DEG shows a resonance when the Fermi circles kiss and overlap, note that there is no dampening due to pseudo-spin overlap. For SLG, the pseudo-spins at the intersections become orthogonal when $Q=0$, which dampens the conductance and removes the divergence at $Q=0$. For BLG, the pseudo-spins at the intersection are orthogonal when $Q=0,2 \bar{k}_{\mathrm{F}}$, but become parallel as $Q \rightarrow \sqrt{2} \bar{k}_{\mathrm{F}}$, which is why the BLG curve touches the 2DEG curve at this point. 


\subsubsection{Molybdenum disulphide}

The semi-conductor $\mathrm{MoS}_{2}$ has the energy-momentum relation

$$
\varepsilon_{\gamma, \mathbf{k}, \sigma}^{(\operatorname{mos})}=\sigma \sqrt{v^{2}\|\mathbf{\Pi}(\mathbf{k}, \mathbf{B})\|^{2}+v^{2} \hbar^{2} k_{\Delta}^{2}}
$$

and the pseudo-spin eigenstates for each valley are

$$
|\sigma\rangle_{\mathbf{K}, \boldsymbol{\Pi}}^{(\operatorname{mos})}=\frac{1}{\sqrt{2}}\left(\begin{array}{c}
\sqrt{\frac{1+\sigma \zeta_{\Pi}}{2}} \mathrm{e}^{-i \theta_{\boldsymbol{\Pi}} / 2} \\
\sigma \sqrt{\frac{1-\sigma \zeta_{\boldsymbol{\Pi}}}{2}} \mathrm{e}^{i \theta_{\boldsymbol{\Pi}} / 2}
\end{array}\right) \quad|\sigma\rangle_{\mathbf{K}^{\prime}, \boldsymbol{\Pi}}^{(\mathrm{mos})}=\frac{1}{\sqrt{2}}\left(\begin{array}{l}
\sqrt{\frac{1+\sigma \zeta_{\Pi}}{2}} \mathrm{e}^{-i\left(\pi-\theta_{\boldsymbol{\Pi}}\right) / 2} \\
\sigma \sqrt{\frac{1-\sigma \zeta_{\Pi}}{2}} \mathrm{e}^{i\left(\pi-\theta_{\boldsymbol{\Pi}}\right) / 2}
\end{array}\right) .
$$

One will notice that the pseudo-spin eigenstates do not lie in the $x y$ plane. This allows for more complexity when pseudo-spin is not conserved in tunnelling. To stay within the scope of this work, we enforce pseudo-spin conservation $\left(\tau=\frac{1}{\sqrt{2}} \tau_{0} \sigma_{0}\right)$, and calculate the linear magneto-tunnelling conductance between two $n$ doped layers of $\mathrm{MoS}_{2}$

$$
\begin{aligned}
\frac{G_{n \leftrightarrow n}^{(\operatorname{mos})}(0)}{G_{0}}= & \frac{\Theta(Q-\Delta) \Theta\left(2 \bar{k}_{\mathrm{F}}-Q\right)}{4} \\
& \times\left\{\sqrt{\frac{4 \bar{k}_{\mathrm{F}}^{2}-Q^{2}}{Q^{2}-\Delta^{2}}}\left[\sqrt{\left(1+\zeta_{k_{\mathrm{F}}^{(1)}}\right)\left(1+\zeta_{k_{\mathrm{F}}^{(2)}}\right)}+\sqrt{\left(1-\zeta_{k_{\mathrm{F}}^{(1)}}\right)\left(1-\zeta_{k_{\mathrm{F}}^{(2)}}\right)}\right]^{2}\right. \\
& \left.+\sqrt{\frac{Q^{2}-\Delta^{2}}{4 \bar{k}_{\mathrm{F}}^{2}-Q^{2}}}\left[\sqrt{\left(1+\zeta_{k_{\mathrm{F}}^{(1)}}\right)\left(1+\zeta_{k_{\mathrm{F}}^{(2)}}\right)}-\sqrt{\left(1-\zeta_{k_{\mathrm{F}}^{(1)}}\right)\left(1-\zeta_{k_{\mathrm{F}}^{(2)}}\right)}\right]^{2}\right\}
\end{aligned}
$$

For tunnelling between $n$ and $p$ doped layers, the linear magneto-tunnelling conductance is

$$
\begin{aligned}
\frac{G_{n \rightarrow p}^{(\operatorname{mos})}(0)}{G_{0}}= & \frac{\Theta(Q-\Delta) \Theta\left(2 \bar{k}_{\mathrm{F}}-Q\right)}{4} \\
& \times\left\{\sqrt{\frac{4 \bar{k}_{\mathrm{F}}^{2}-Q^{2}}{Q^{2}-\Delta^{2}}}\left[\sqrt{\left(1+\zeta_{k_{\mathrm{F}}^{(1)}}\right)\left(1-\zeta_{k_{\mathrm{F}}^{(2)}}\right)}-\sqrt{\left(1-\zeta_{k_{\mathrm{F}}^{(1)}}\right)\left(1+\zeta_{k_{\mathrm{F}}^{(2)}}\right)}\right]^{2}\right. \\
& \left.+\sqrt{\frac{Q^{2}-\Delta^{2}}{4 \bar{k}_{\mathrm{F}}^{2}-Q^{2}}}\left[\sqrt{\left(1+\zeta_{k_{\mathrm{F}}^{(1)}}\right)\left(1-\zeta_{k_{\mathrm{F}}^{(2)}}\right)}+\sqrt{\left(1-\zeta_{k_{\mathrm{F}}^{(1)}}\right)\left(1+\zeta_{k_{\mathrm{F}}^{(2)}}\right)}\right]^{2}\right\}
\end{aligned}
$$

If the densities in each layer are equal, one finds

$$
\frac{G_{n \leftrightarrow n}^{(\mathrm{mos})}(0)}{G_{0}}=\left(\frac{\sqrt{4 \bar{k}_{\mathrm{F}}^{2}-Q^{2}}}{Q}+\frac{\zeta_{\bar{k}_{\mathrm{F}}}^{2} Q}{\sqrt{4 \bar{k}_{\mathrm{F}}^{2}-Q^{2}}}\right) \Theta\left(2 \bar{k}_{\mathrm{F}}-Q\right) .
$$

We have denoted $\zeta_{\bar{k}_{\mathrm{F}}}=k_{\Delta} / \sqrt{k_{\mathrm{F}}^{2}+k_{\Delta}^{2}}$, which is a measure of how close the Fermi energy $v \hbar k_{\mathrm{F}}$ is to the band gap $v \hbar k_{\Delta}$. When the band gap is very small, $\frac{\bar{k}_{\mathrm{F}}}{k_{\Delta}}$ is very 


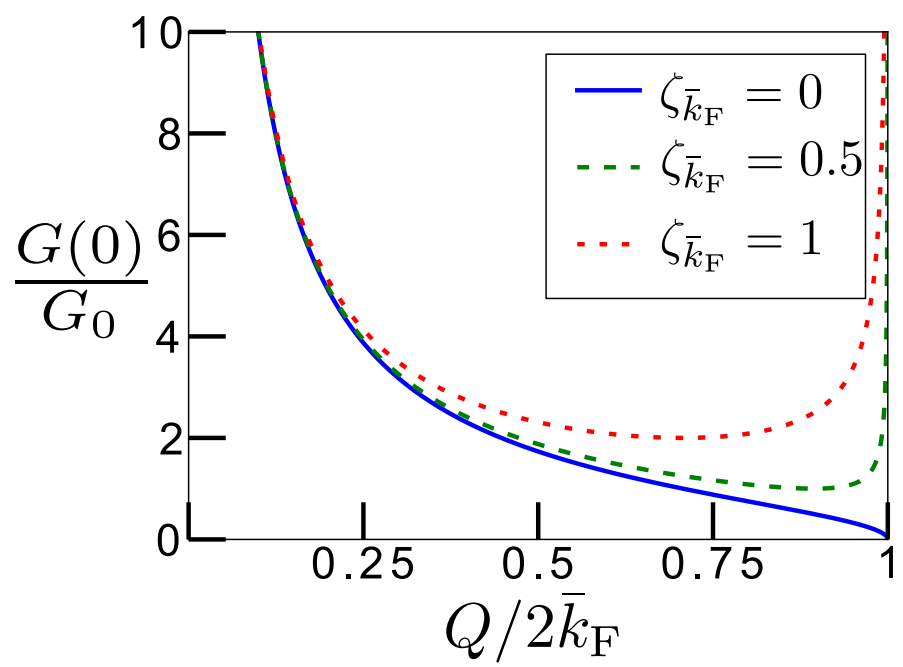

Figure 4.8: The linear magneto-tunnelling conductance for two $n$ doped layers of $\mathrm{MoS}_{2}$. One can see the transition from SLG to 2DEG as $\zeta_{\bar{k}_{\mathrm{F}}}$ transitions from 0 to 1.

large, so $\zeta_{\bar{k}_{\mathrm{F}}} \approx 0$ and one obtains the result found for SLG. For a Fermi energy close to the band gap $\frac{k_{\mathrm{F}}}{k_{\Delta}} \approx 0$, so $\zeta_{\bar{k}_{\mathrm{F}}} \approx 1$ and obtains the result for 2DEG. This shows how one can change the Fermi energy to switch on chiral characteristics. One can see the transition from SLG to 2DEG characteristics for equal densities in Fig. 4.8.

One will also find

$$
\frac{G_{n \leftrightarrow p}^{(\operatorname{mos})}(0)}{G_{0}}=\frac{\left(1-\zeta_{\bar{k}_{\mathrm{F}}}^{2}\right) Q}{\sqrt{4 \bar{k}_{\mathrm{F}}^{2}-Q^{2}}} \Theta\left(2 \bar{k}_{\mathrm{F}}-Q\right) .
$$

When $\frac{k_{\mathrm{F}}}{k_{\Delta}}$ is very large, one obtains the result found for SLG. However, when $\frac{k_{\mathrm{F}}}{k_{\Delta}} \approx 0$, the pseudo-spin in the $n$ layer points along $+z$ and the pseudo-spin in the $p$ layer points along $-z$. This means the magneto-tunnelling conductance vanishes due to zero pseudo-spin overlap. 


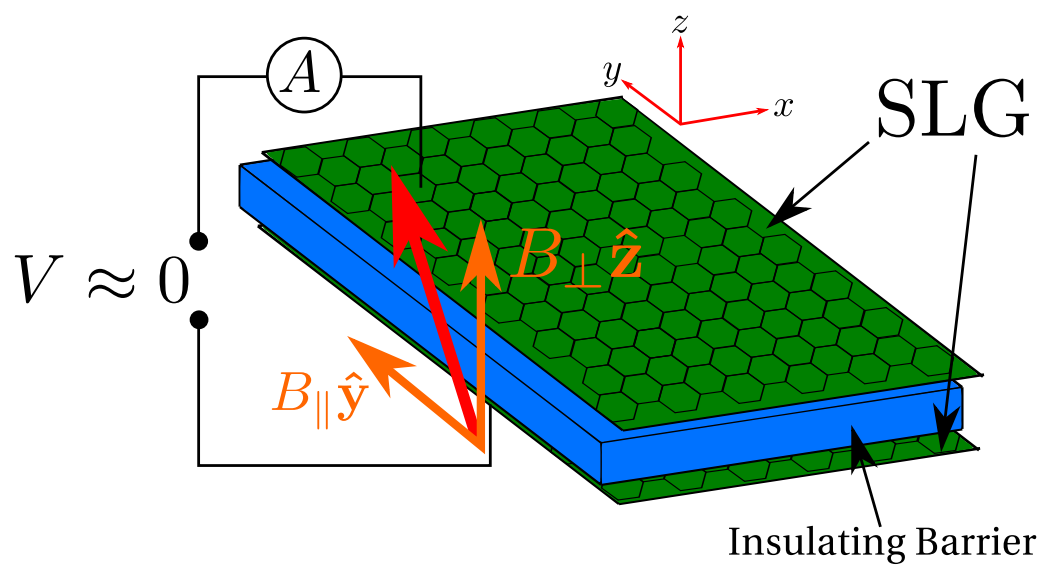

Figure 4.9: Two layers of SLG separated by a barrier, with $V \approx 0$. A tilted magnetic field, $\mathbf{B}=B_{\|} \hat{\mathbf{y}}+B_{\perp} \hat{\mathbf{z}}$ is applied across the systems and barrier. The red arrow represents the magnetic field, with its components shown in orange. This magnetic field produces discrete Landau level states and energies, with the cyclotron orbit guiding centres shifted by the in-plane magnetic field component. This shift in the orbit guiding centres creates oscillations in the wave function overlap between the SLG layers, as a function of the in-plane magnetic field.

\subsubsection{Experimental requirements}

To observe the full extent of the magneto-tunnelling characteristics discussed in this work requires sufficiently large magnetic fields to shift the Fermi circles in kinetic momentum space, through to separation. This leads to the condition $d \geq 2 \bar{k}_{\mathrm{F}} \ell_{B_{\|}^{(\operatorname{Max})}}^{2}$, which ensures that the largest applied magnetic field will separate the Fermi circles completely. For the case of two layers of SLG, with equal densities $n=g_{\mathrm{s}} g_{\mathrm{v}} \bar{k}_{\mathrm{F}}^{2} /(4 \pi)$ in the two layers, we find

$$
B_{\|}^{(\operatorname{Max})} \geq \frac{2 \pi \hbar}{e} \sqrt{\frac{4}{g_{\mathrm{s}} g_{\mathrm{v}}} \frac{n}{\pi d^{2}}} \approx 20 \mathrm{~T} \times \frac{\sqrt{n\left[10^{10} \mathrm{~cm}^{-2}\right]}}{d[\mathrm{~nm}]} .
$$

In recently made Van der Waals heterostructures, made from stacking SLG/h-BN/SLG, a typical tunnelling barrier will be made from four to nine layers of h-BN. The thickness of one layer of h-BN is $0.33 \mathrm{~nm}$. With densities on the order of $10^{10} \mathrm{~cm}^{-2}$, one would need an in-plane magnetic field of at most $20 \mathrm{~T}$.

\subsection{Landau levels in single layer graphene}

In this section, we look at the linear magneto-tunnelling conductance between two layers of SLG when the magnetic field is titled in the $z y$ plane, i.e. $\mathbf{B}=B_{\|} \hat{\mathbf{y}}+B_{\perp} \hat{\mathbf{z}}$, as shown in Fig. 4.9. We enforce momentum conservation, using the tunnelling matrix found in Eq. (4.2). To calculate Eq. (3.20) for two layers of SLG in a tilted magnetic field, one needs to know the eigenstates and energies of SLG in a tilted 
magnetic field. This is an extension of Ref. [24], which has been briefly reviewed in section 3.2 .2

Using the Landau level ladder operators defined by $a^{ \pm}=\ell_{B_{\perp}}\left(\Pi_{x} \pm i \Pi_{y}\right) /(\sqrt{2} \hbar)$, the single-particle Hamiltonians for the $\mathbf{K}$ and $\mathbf{K}^{\prime} \equiv-\mathbf{K}$ valleys of SLG are given by

$$
\mathcal{H}_{ \pm \mathbf{K}}^{(\mathrm{slg})}(\mathbf{B})= \pm \sqrt{2} \frac{\hbar v}{\ell_{B_{\perp}}}\left(\begin{array}{cc}
0 & a^{\mp} \\
a^{ \pm} & 0
\end{array}\right)
$$

As in section 3.2.2, we choose the Landau gauge $\mathbf{A}=\left(-y B_{\perp}+z B_{\|}, 0,0\right)$. The energy eigenvalues of $\mathcal{H}_{ \pm \mathbf{K}}^{\text {(slg) }}(\mathbf{B})$ are found to be $\varepsilon_{\gamma, \sigma, \nu}=\sigma \hbar v \sqrt{2 \nu} / \ell_{B_{\perp}}$, where $\nu=0,1, \ldots$, and the corresponding eigenstates in the $\mathbf{K}$ and $\mathbf{K}^{\prime}$ valleys are

$$
\begin{aligned}
\left|\nu, \sigma, \kappa_{x}\right\rangle_{\mathbf{K}} & =\frac{1}{\sqrt{2}}\left(\begin{array}{c}
\sigma\left|\nu-1, \kappa_{x}\right\rangle \\
\left|\nu, \kappa_{x}\right\rangle
\end{array}\right) \text { for } \nu>0,\left|0, \kappa_{x}\right\rangle_{\mathbf{K}}=\left(\begin{array}{c}
0 \\
\left|0, \kappa_{x}\right\rangle
\end{array}\right) \\
\left|\nu, \sigma, \kappa_{x}\right\rangle_{\mathbf{K}^{\prime}} & =\frac{1}{\sqrt{2}}\left(\begin{array}{c}
\left|\nu, \kappa_{x}\right\rangle \\
\sigma\left|\nu-1, \kappa_{x}\right\rangle
\end{array}\right) \text { for } \nu>0\left|0, \kappa_{x}\right\rangle_{\mathbf{K}^{\prime}}=\left(\begin{array}{c}
\left|0, \kappa_{x}\right\rangle \\
0
\end{array}\right) .
\end{aligned}
$$

Here the real-space Landau level eigenstates satisfy $a^{+} a^{-}\left|\nu, k_{x}\right\rangle=\nu\left|\nu, k_{x}\right\rangle$, with the wave number $\kappa_{x}=k_{x}+\frac{z}{\ell_{B_{\|}}^{2}}$ determining the cyclotron-orbit guiding-centre position in $y$ direction.

\subsubsection{Linear magneto-tunnelling conductance in tilted mag- netic field}

One can now write the linear magneto-tunnelling conductance between two layers of SLG in a tilted magnetic field as

$$
\begin{aligned}
G^{(\mathrm{LLg})}(0)= & \frac{g_{\mathrm{s}} g_{\mathrm{v}} e^{2}}{\hbar} \frac{A}{\hbar^{2} v^{2}} \sqrt{\nu_{\mathrm{F}}^{(1)}\left(\nu_{\mathrm{F}}^{(1)}+\Delta \nu_{\mathrm{F}}\right)} \sum_{\nu_{1}, \nu_{2}=1}^{\infty} \delta\left(\nu_{\mathrm{F}}^{(1)}-\nu_{1}\right) \delta\left(\nu_{\mathrm{F}}^{(1)}+\Delta \nu_{\mathrm{F}}-\nu_{2}\right) \\
& \times\left[\left|\tau_{0} F_{\nu_{1} \nu_{2}}^{(0)}(\xi)+\tau_{x} F_{\nu_{1} \nu_{2}}^{(x)}(\xi)\right|^{2}+\left|\tau_{y} F_{\nu_{1} \nu_{2}}^{(y)}(\xi)+\tau_{z} F_{\nu_{1} \nu_{2}}^{(z)}(\xi)\right|^{2}\right],
\end{aligned}
$$

We denote the Landau level at the Fermi energy in layer $j$ by $\nu_{\mathrm{F}}^{(j)}$, and $\Delta \nu_{\mathrm{F}}=$ $\nu_{\mathrm{F}}^{(2)}-\nu_{\mathrm{F}}^{(1)}$. We have not included $\nu^{(1)}=0$ or $\nu^{(2)}=0$ contribution in the sum because of the vanishing pre-factor, however, for electrons with finite life-times there will be a contribution. We have defined

$$
F_{\nu_{1} \nu_{2}}^{(\eta)}(\xi)={ }_{\mathbf{K}}\left\langle\nu_{1}, \sigma_{\mathrm{F}}^{(1)}, \kappa_{x}^{(1)}\left|\sigma_{\eta}\right| \nu_{2}, \sigma_{\mathrm{F}}^{(2)}, \kappa_{x}^{(2)}\right\rangle_{\mathbf{K}}
$$


where $\sigma_{\eta}$ are Pauli matrices for $\eta=0, x, y, z$, and $\sigma_{\mathrm{F}}^{(m)}=\operatorname{Sign}\left(\varepsilon_{\mathrm{F}}^{(m)}\right)$. One can then calculate the following

$$
\begin{aligned}
& F_{\nu_{1} \nu_{2}}^{(0)}(\xi)= \frac{1}{2}\left(\frac{\nu_{<} !}{\nu_{>} !}\right)^{\frac{1}{2}}\left(\frac{\xi^{2}}{2}\right)^{\frac{\nu_{>}-\nu_{<}}{2}} \mathrm{e}^{-\frac{\xi^{2}}{4}} \\
& \times\left[L_{\nu_{<}}^{\nu_{>}-\nu_{<}}\left(\frac{\xi^{2}}{2}\right) \pm \sqrt{\frac{\nu_{>}}{\nu_{<}}} L_{\nu<-1}^{\nu_{>}-\nu_{<}}\left(\frac{\xi^{2}}{2}\right)\right] \\
& F_{\nu_{1} \nu_{2}}^{(x)}(\xi)=-\frac{1}{2}\left(\frac{\nu_{<} !}{\nu_{>} !}\right)^{\frac{1}{2}}\left(\frac{\xi^{2}}{2}\right)^{\frac{\nu_{>}-\nu_{<}-1}{2}} \mathrm{e}^{-\frac{\xi^{2}}{4}} \\
& \times\left[\sqrt{\nu_{>}} L_{\nu<}^{\nu_{>}-\nu_{<}-1}\left(\frac{\xi^{2}}{2}\right) \pm \frac{\xi^{2}}{2 \sqrt{\nu_{<}}} L_{\nu_{<}-1}^{\nu_{>}-\nu_{<}+1}\left(\frac{\xi^{2}}{2}\right)\right] \\
& F_{\nu_{1} \nu_{2}}^{(y)}(\xi)=-\frac{i}{2}\left(\frac{\nu_{<} !}{\nu_{>} !}\right)^{\frac{1}{2}}\left(\frac{\xi^{2}}{2}\right)^{\frac{\nu_{>}-\nu_{<}-1}{2}} \mathrm{e}^{-\frac{\xi^{2}}{4}} \\
& \times\left[\sqrt{\nu_{>}} L_{\nu_{<}}^{\nu_{>}-\nu_{<}-1}\left(\frac{\xi^{2}}{2}\right) \mp \frac{\xi^{2}}{2 \sqrt{\nu_{<}}} L_{\nu_{<}-1}^{\nu_{>}-\nu_{<}+1}\left(\frac{\xi^{2}}{2}\right)\right] \\
& \frac{1}{2}\left(\frac{\nu_{<} !}{\nu_{>} !}\right)^{\frac{1}{2}}\left(\frac{\xi^{2}}{2}\right)^{\frac{\nu_{>}-\nu_{<}}{2}} \mathrm{e}^{-\frac{\xi^{2}}{4}} \\
& F_{\nu_{1} \nu_{2}}^{(z)}(\xi)=\left[L_{\nu_{<}}^{\nu_{>}-\nu_{<}}\left(\frac{\xi^{2}}{2}\right) \mp \sqrt{\frac{\nu_{>}}{\nu_{<}}} L_{\nu_{<}-1}^{\nu_{>}-\nu_{<}}\left(\frac{\xi^{2}}{2}\right)\right]
\end{aligned}
$$

where the upper (lower) $+/-$ sign is used for tunnelling between two $n$ doped layers (an $n$ and $p$ doped layer). As defined in section 3.2.2. $\nu_{<(>)}=\min (\max )\left\{\nu, \nu^{\prime}\right\}$, $\xi=\left(d / \ell_{B_{\perp}}\right)\left(B_{\|} / B_{\perp}\right)$, and $L_{n}^{n^{\prime}}(\cdot)$ is the generalized Laguerre polynomial. When $\nu_{1}=\nu_{2} \equiv \nu$, we simplify the notation to

$$
\begin{aligned}
\left.F_{\nu \nu}^{(0)}(\xi)\right|_{n \rightarrow n} & \left.\equiv F_{\nu \nu}^{(z)}(\xi)\right|_{n \rightarrow p}=\mathcal{F}_{\nu}^{(+)}(\xi) \\
\left.F_{\nu \nu}^{(x)}(\xi)\right|_{n \rightarrow n} & \left.\equiv i F_{\nu \nu}^{(y)}(\xi)\right|_{n \rightarrow p}=\mathcal{F}_{\nu}^{(\perp)}(\xi) \\
\left.F_{\nu \nu}^{(y)}(\xi)\right|_{n \rightarrow n} & \left.\equiv F_{\nu \nu}^{(x)}(\xi)\right|_{n \rightarrow p}=0 \\
\left.F_{\nu \nu}^{(z)}(\xi)\right|_{n \rightarrow n} & \left.\equiv F_{\nu \nu}^{(0)}(\xi)\right|_{n \rightarrow p}=\mathcal{F}_{\nu}^{(-)}(\xi)
\end{aligned}
$$

with the definitions

$$
\begin{aligned}
& \mathcal{F}_{\nu}^{( \pm)}(\xi)=\frac{1}{2} \mathrm{e}^{-\frac{\xi^{2}}{4}}\left[L_{\nu}^{0}\left(\frac{\xi^{2}}{2}\right) \pm L_{\nu-1}^{0}\left(\frac{\xi^{2}}{2}\right)\right] \\
& \mathcal{F}_{\nu}^{(\perp)}(\xi)=-\mathrm{e}^{-\frac{\xi^{2}}{4}} \sqrt{\frac{\xi^{2}}{2 \nu}} L_{\nu-1}^{1}\left(\frac{\xi^{2}}{2}\right) .
\end{aligned}
$$


One can then write the linear magneto-tunnelling conductance as

$$
\begin{aligned}
G_{n \leftrightarrow n}^{(\mathrm{LLg})}(0)= & \frac{g_{\mathrm{s}} g_{\mathrm{v}} e^{2}}{\hbar} \frac{A}{\hbar^{2} v^{2}} \nu_{\mathrm{F}} \sum_{\nu_{1}, \nu_{2}=1}^{\infty} \delta\left(\nu_{\mathrm{F}}-\nu_{1}\right) \delta\left(\nu_{\mathrm{F}}-\nu_{2}\right) \\
& \times\left[\left|\tau_{0} \mathcal{F}_{\nu_{1}}^{(+)}(\xi)+\tau_{\perp} \mathcal{F}_{\nu_{1}}^{(\perp)}(\xi)\right|^{2}+\left|\tau_{z} \mathcal{F}_{\nu_{1}}^{(-)}(\xi)\right|^{2}\right], \\
G_{n \leftrightarrow p}^{(\mathrm{LLg})}(0)= & \frac{g_{\mathrm{s}} g_{\mathrm{v}} e^{2}}{\hbar} \frac{A}{\hbar^{2} v^{2}} \nu_{\mathrm{F}} \sum_{\nu_{1}, \nu_{2}=1}^{\infty} \delta\left(\nu_{\mathrm{F}}-\nu_{1}\right) \delta\left(\nu_{\mathrm{F}}-\nu_{2}\right) \\
& \times\left[\left|\tau_{0} \mathcal{F}_{\nu_{1}}^{(-)}(\xi)\right|^{2}+\left|\tau_{\|} \mathcal{F}_{\nu_{1}}^{(\perp)}(\xi)+\tau_{z} \mathcal{F}_{\nu_{1}}^{(+)}(\xi)\right|^{2}\right] .
\end{aligned}
$$

One can see plots of Eqs. 4.64 in Fig. 4.10, as a function of $\xi$. One will see that increasing $B_{\|}$will result in oscillations in the magneto-tunnelling conductance. This is because $B_{\|}$determines the shift in the cyclotron orbit guiding centres, which shifts the wave function overlap between the orbits in each layer. These oscillations also happen in the non-chiral tunnelling shown in section 3.2.2, however the pseudospin tunnel-coupled structure allows for more possible oscillations in wave function overlap.

\subsubsection{Effect of a perpendicular magnetic field}

In the limit that $\mathbf{B}=B_{\perp} \hat{\mathbf{z}}$, one finds that $\xi=0$. In this case, the linear magnetotunnelling conductance between two SLG layers is

$$
\begin{aligned}
G_{n \leftrightarrow n}^{(\mathrm{LLg})}(0)= & \frac{g_{\mathrm{s}} g_{\mathrm{v}} \mathrm{e}^{2}}{\hbar} \frac{A}{\hbar^{2} v^{2}} \sqrt{\nu_{\mathrm{F}}^{(1)}\left(\nu_{\mathrm{F}}^{(1)}+\Delta \nu_{\mathrm{F}}\right)} \sum_{\nu_{1}, \nu_{2}=1}^{\infty} \delta\left(\nu_{\mathrm{F}}^{(1)}-\nu_{1}\right) \delta\left(\nu_{\mathrm{F}}^{(1)}+\Delta \nu_{\mathrm{F}}-\nu_{2}\right) \\
& \times\left[\left|\tau_{0} \delta_{0, \Delta \nu_{\mathrm{F}}}+\tau_{x} \frac{\delta_{-1, \Delta \nu_{\mathrm{F}}}+\delta_{1, \Delta \nu_{\mathrm{F}}}}{2}\right|^{2}+\left|\tau_{y}\right|^{2} \frac{\delta_{-1, \Delta \nu_{\mathrm{F}}}-\delta_{1, \Delta \nu_{\mathrm{F}}}}{4}\right], \quad(4.66 \mathrm{a}) \\
G_{n \leftrightarrow p}^{(\mathrm{LLg})}(0)= & \frac{g_{\mathrm{s}} g_{\mathrm{v}} \mathrm{e}^{2}}{\hbar} \frac{A}{\hbar^{2} v^{2}} \sqrt{\nu_{\mathrm{F}}^{(1)}\left(\nu_{\mathrm{F}}^{(1)}+\Delta \nu_{\mathrm{F}}\right)} \sum_{\nu_{1}, \nu_{2}=1}^{\infty} \delta\left(\nu_{\mathrm{F}}^{(1)}-\nu_{1}\right) \delta\left(\nu_{\mathrm{F}}^{(1)}+\Delta \nu_{\mathrm{F}}-\nu_{2}\right) \\
& \times\left[\left|\tau_{x}\right|^{2} \frac{\delta_{-1, \Delta \nu_{\mathrm{F}}}+\delta_{1, \Delta \nu_{\mathrm{F}}}}{4}+\left|\tau_{y} \frac{\delta_{-1, \Delta \nu_{\mathrm{F}}}-\delta_{1, \Delta \nu_{\mathrm{F}}}}{2}-i \tau_{z} \delta_{0, \Delta \nu_{\mathrm{F}}}\right|^{2}\right] . \quad(4.66 \mathrm{~b})
\end{aligned}
$$

There are resonances in tunnelling between the Landau levels at the Fermi energy. But, the possible resonances are also limited from the pseudo-spin structure of the tunnelling matrix. For tunnelling between two $n$-doped layers, pseudo-spin conservation would mean that a resonance could occur at equal Fermi energies. However, if the barrier causes pseudo-spin flips about the $x$ or $y$ axes in the tunnelling process, this will allow adjacent SLG states to overlap, causing a resonance when the Fermi energies are slightly mismatched $\left(\Delta_{\mathrm{F}}= \pm 1\right)$.

In a system with disorder, the resonances will have a finite width and height. One can use the Born approximation so that the spectral functions become Lorentzian. 

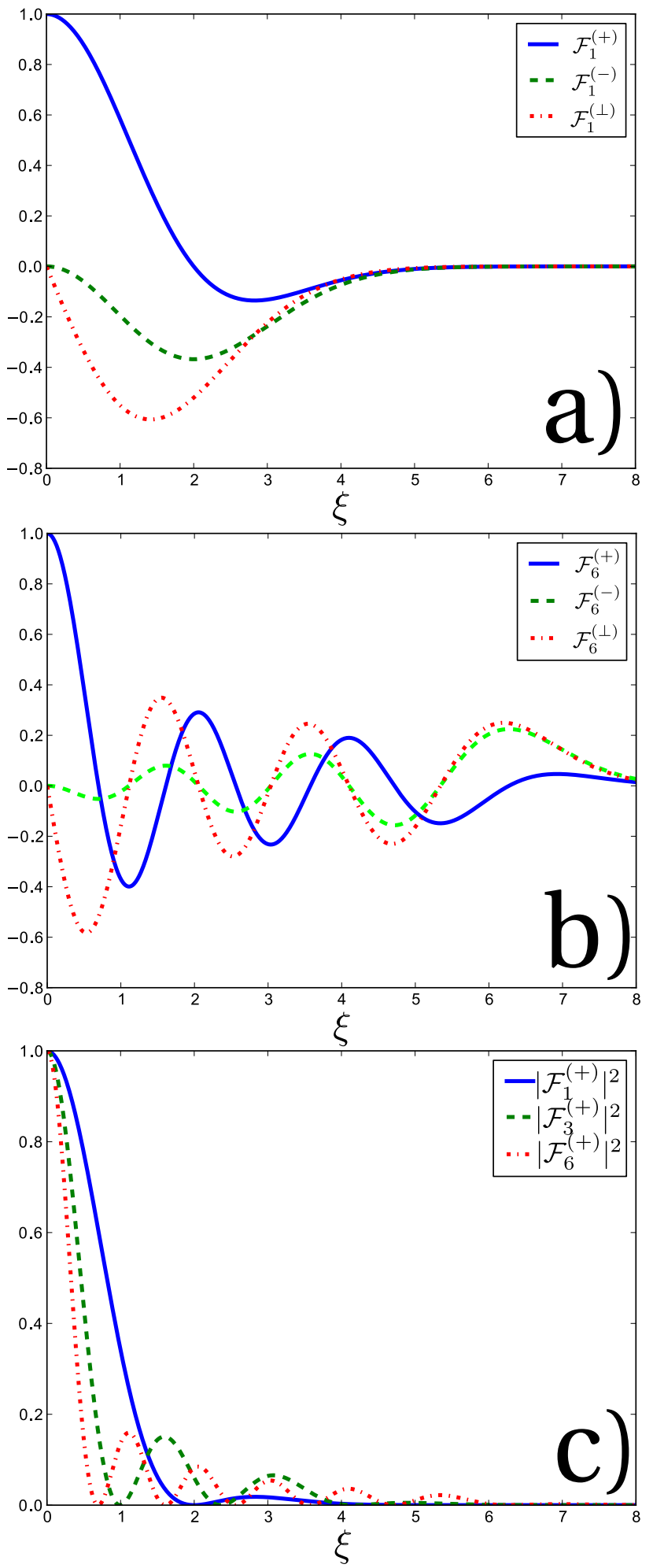

Figure 4.10: a) and b) are plots of the form factors in Eqs. (4.64) for Landau Fermi levels $\nu_{\mathrm{F}}=1,6$ as a function of $\xi=\left(d / \ell_{B_{\perp}}\right)\left(B_{\|} / B_{\perp}\right)$. c) shows shows $\left|\mathcal{F}^{(+)}(\xi)\right|^{2}$ for $\nu_{\mathrm{F}}=1,3,6$, which is relevant when pseudo-spin is conserved $\left(\tau=\frac{1}{\sqrt{2}} \tau_{0} \sigma_{0}\right)$. The oscillatory behaviour is due to the shift in orbit guiding centres in each layer due to $B_{\|} \cdot$ 
This suggests that the resonance in tunnelling between the Landau levels shown here by the delta functions, will have a finite width and peak determined by the electron life-time of the system.

It is useful to notice that the density in each layer is related to $\nu_{\mathrm{F}}$ via summation of states

$$
n=\frac{g_{\mathrm{s}} g_{\mathrm{v}}}{A} \sum_{k_{x}} \sum_{\nu=0}^{\nu_{\mathrm{F}}} \approx \frac{g_{\mathrm{s}} g_{\mathrm{v}}}{2 \pi} \frac{e B_{\perp}}{\hbar}\left(\nu_{\mathrm{F}}+1\right) .
$$

This relation between $n$ and $\nu_{\mathrm{F}}$ shows that $\Delta \nu_{\mathrm{F}} \propto n^{(2)}-n^{(1)}$. This makes it explicitly clear how changing the density in each layer will shift the Fermi Landau levels.

In particular, when the densities in each layer are equal $\Delta \nu_{\mathrm{F}}=0$, and one will find only a contribution from the pseudo-spin conserving component of the tunnelling matrix $\tau_{0}$ or the pseudo-spin flip component $\tau_{z}$

$$
\begin{aligned}
& G_{n \leftrightarrow n}^{(\mathrm{LLg})}=\frac{g_{\mathrm{s}} g_{\mathrm{v}} \mathrm{e}^{2}}{\hbar} \frac{A\left|\tau_{0}\right|^{2}}{\hbar^{2} v^{2}} \nu_{\mathrm{F}} \sum_{\nu_{1}, \nu_{2}} \delta\left(\nu_{\mathrm{F}}-\nu_{1}\right) \delta\left(\nu_{\mathrm{F}}-\nu_{2}\right), \\
& G_{n \leftrightarrow p}^{(\mathrm{LLg})}=\frac{g_{\mathrm{s}} g_{\mathrm{v}} \mathrm{e}^{2}}{\hbar} \frac{A\left|\tau_{z}\right|^{2}}{\hbar^{2} v^{2}} \nu_{\mathrm{F}} \sum_{\nu_{1}, \nu_{2}} \delta\left(\nu_{\mathrm{F}}-\nu_{1}\right) \delta\left(\nu_{\mathrm{F}}-\nu_{2}\right) .
\end{aligned}
$$

Since these delta functions will be Lorentzian for systems with disorder, one will be able to probe the $\tau_{0}$ and $\tau_{z}$ components for any barrier, when both layers have equal density. If one knows the life-time for the SLG systems, one should be able to estimate $\left|\tau_{0}\right|^{2}$ and $\left|\tau_{0}\right|^{2}$ from experiment. 


\section{Chapter 5}

\section{Magneto-tunnelling as a spectroscopic tool}

In this chapter, we investigate how magneto-tunnelling can be used as a spectroscopic tool. We start by looking at how measuring the linear magneto-tunnelling conductance for an in-plane and titled magnetic field may allow one to measure the tunnel-coupling between two parallel SLG layers, which could provide insight into the properties of the double layer SLG system. Then we investigate parallel layers of SLG and BLG in an in-plane magnetic field, where we find conditions that could lead to a valley polarised current. We discuss the effect of electron-electron interactions in SLG, and how they will alter the linear magneto-tunnelling for an in-plane magnetic field in a double layer SLGs system. We use a mean field theory (Hartree-Fock) approximation to model the interactions. In this case the interactions renormalise the velocity, which changes the density of states at the Fermi level of each layer. This results in a dampening of the tunnelling-current magnitude, allowing one to observe the effect of electron-electron interactions. Last in this chapter, we investigate how an in-plane electric field would alter the linear magneto-tunnelling conductance in a double layer SLG system, for an in-plane magnetic field. We find that one can observe the effects of an in-plane electric field through resonance shifts in the linear magneto-tunnelling conductance.

\subsection{Experimental measurement of tunnel-coupling}

In this section, we focus on showing how one can measure the zero bias magnetotunnelling conductance to obtain amplitudes of the tunnelling matrix parameters, $\left|\tau_{\alpha}\right|^{2}$. Then we discuss how these parameters could be dependent on the lattice structure for a van der Waals heterostructure.

In the previous chapter, we have shown that for $\mathbf{B}=B_{\perp} \hat{\mathbf{z}}$, the magneto-tunnelling conductance between two layers of SLG with equal densities could allow one to estimate $\left|\tau_{0}\right|^{2}$ and $\left|\tau_{z}\right|^{2}$ from experiment. To measure $\left|\tau_{\|}\right|^{2},\left|\tau_{\perp}\right|^{2},\left|\tau_{z}\right|^{2}$, one can look at the magneto-tunnelling conductance for an in-plane magnetic field $\mathbf{B}=B_{\|} \hat{\mathbf{b}}$.

It is possible rearrange the Eq. 4.38 into a polynomial of the form

$$
F\left(Q, \bar{k}_{\mathrm{F}}, \Delta\right)=\frac{2 \pi \hbar G_{n \leftrightarrow n}^{(\mathrm{slg})}(0)}{g_{\mathrm{s}} g_{\mathrm{v}} \mathrm{e}^{2}} Q^{2} \sqrt{\left(4 \bar{k}_{\mathrm{F}}^{2}-Q^{2}\right)\left(Q^{2}-\Delta^{2}\right)}
$$


where

$$
F\left(Q, \bar{k}_{\mathrm{F}}, \Delta\right) \equiv-c_{1} Q^{4}+c_{2} Q^{2} \bar{k}_{\mathrm{F}}^{2}-c_{3} Q^{2} \Delta^{2}+c_{4} \bar{k}_{\mathrm{F}}^{2} \Delta^{2} .
$$

We have defined the coefficients as

$$
\begin{aligned}
& c_{1} \equiv \frac{A\left(\left|\tau_{0}\right|^{2}-\left|\tau_{z}\right|^{2}\right)}{\hbar^{2} v^{2}}, c_{2} \equiv 4 \frac{A\left(\left|\tau_{0}\right|^{2}+\left|\tau_{\|}\right|^{2}\right)}{\hbar^{2} v^{2}}, \\
& c_{3} \equiv \frac{A\left(\left|\tau_{\perp}\right|^{2}+\left|\tau_{z}\right|^{2}\right)}{\hbar^{2} v^{2}}, c_{4} \equiv 4 \frac{A\left(\left|\tau_{\perp}\right|^{2}-\left|\tau_{\|}\right|^{2}\right)}{\hbar^{2} v^{2}} .
\end{aligned}
$$

In practice, one can perform a least squares fit to this polynomial and obtain an estimate for the coefficients. This can be done by first choosing the densities in each layer to be equal, which allows one to make a measurement of $G_{n \leftrightarrow n}^{(\mathrm{slg})}(0)$ when $\Delta=0$, then using a least squares allows one to obtain $c_{1}$ and $c_{2}$. Knowing $c_{1}$ and $c_{2}$, another measurement of $G_{n \leftrightarrow n}^{(\mathrm{slg})}(0)$ where $\Delta \neq 0$ will make it possible to obtain $c_{3}$ and $c_{4}$.

According to theory, these coefficients are not independent due to their relation with the tunnelling matrix. We can show this in a matrix format, where our system of equations has the form

$$
\frac{A}{\hbar^{2} v^{2}}\left(\begin{array}{cccc}
1 & -1 & 0 & 0 \\
4 & 0 & 4 & 0 \\
0 & 1 & 0 & 1 \\
0 & 0 & -4 & 4
\end{array}\right)\left(\begin{array}{l}
\left|\tau_{0}\right|^{2} \\
\left|\tau_{z}\right|^{2} \\
\left|\tau_{\|}\right|^{2} \\
\left|\tau_{\perp}\right|^{2}
\end{array}\right)=\left(\begin{array}{l}
c_{1} \\
c_{2} \\
c_{3} \\
c_{4}
\end{array}\right)
$$

From a simple calculation, this matrix only has rank three, so there are only three independent equations with four unknowns. However, it is possible solve for an upper bound on the tunnelling matrix element amplitudes

$$
\frac{A}{\hbar^{2} v^{2}} \operatorname{Tr}\left[\tau^{\dagger} \tau\right]=\frac{1}{4} c_{2}+c_{3}
$$

Also, we find that $c_{4}$ must depend on $c_{1}, c_{2}$, and $c_{3}$ from the relation

$$
c_{2}+c_{4}=4\left(c_{1}+c_{3}\right)
$$

If this equality is not true for the fitted parameters, then the derived theory is not consistent with experiment. To be clear, this equality allows one to test if the theory is wrong.

If uses magnetic field perpendicular to the double layer system, Eq. 4.65 can be used to independently measure $c_{5}=\frac{A}{\hbar^{2} v^{2}}\left|\tau_{0}\right|^{2}$, and we find

$$
\frac{A}{\hbar^{2} v^{2}}\left(\begin{array}{cccc}
1 & -1 & 0 & 0 \\
4 & 0 & 4 & 0 \\
0 & 1 & 0 & 1 \\
0 & 0 & -4 & 4 \\
1 & 0 & 0 & 0
\end{array}\right)\left(\begin{array}{c}
\left|\tau_{0}\right|^{2} \\
\left|\tau_{z}\right|^{2} \\
\left|\tau_{\|}\right|^{2} \\
\left|\tau_{\perp}\right|^{2}
\end{array}\right)=\left(\begin{array}{c}
c_{1} \\
c_{2} \\
c_{3} \\
c_{4} \\
c_{5}
\end{array}\right)
$$


The rank of this matrix is four, so it is clear that one can always solve for all of the magnitudes of tunnelling matrix parameters $\left|\tau_{\alpha}\right|^{2}$. For any barrier, one can use the following formula

$$
\begin{aligned}
\frac{A}{\hbar^{2} v^{2}}\left|\tau_{0}\right|^{2} & =c_{5}, \\
\frac{A}{\hbar^{2} v^{2}}\left|\tau_{z}\right|^{2} & =-c_{1}+c_{5}, \\
\frac{A}{\hbar^{2} v^{2}}\left|\tau_{\|}\right|^{2} & =\frac{1}{4} c_{2}-c_{5}, \\
\frac{A}{\hbar^{2} v^{2}}\left|\tau_{\perp}\right|^{2} & =c_{1}+c_{3}-c_{5} .
\end{aligned}
$$

A general expression for the magnitudes of tunnelling matrix parameters can be given in terms of the azimuthal angle $\theta_{\mathbf{B}_{\|}} \equiv \arctan \left(B_{\|, y} / B_{\|, x}\right)$ of the in-plane magnetic field,

$$
\begin{gathered}
\left|\tau_{\perp}\left(\theta_{\mathbf{B}_{\|}}\right)\right|^{2}=\frac{\left|\tau_{x}\right|^{2}+\left|\tau_{y}\right|^{2}}{2}+\frac{\left|\tau_{x}\right|^{2}-\left|\tau_{y}\right|^{2}}{2} \cos \left(2 \theta_{\mathbf{B}_{\|}}\right) \\
+\Re\left\{\tau_{x} \tau_{y}^{*}\right\} \sin \left(2 \theta_{\mathbf{B}_{\|}}\right), \\
\left|\tau_{\|}\left(\theta_{\mathbf{B}_{\|}}\right)\right|^{2}=\frac{\left|\tau_{x}\right|^{2}+\left|\tau_{y}\right|^{2}}{2}-\frac{\left|\tau_{x}\right|^{2}-\left|\tau_{y}\right|^{2}}{2} \cos \left(2 \theta_{\mathbf{B}_{\|}}\right) \\
-\Re\left\{\tau_{x} \tau_{y}^{*}\right\} \sin \left(2 \theta_{\mathbf{B}_{\|}}\right) .
\end{gathered}
$$

If a measurement is performed at $\theta_{\mathbf{B}_{\|}}=\frac{\pi}{4}$ and $\theta_{\mathbf{B}_{\|}}=0$, one can obtain the phase difference between $\tau_{x}$ and $\tau_{y}$

$$
\arg \left(\tau_{x} \tau_{y}^{*}\right)=\arccos \left[\frac{\left|\tau_{\perp}\left(\frac{\pi}{4}\right)\right|^{2}-\left|\tau_{\perp}\left(\frac{\pi}{4}\right)\right|^{2}}{2\left|\tau_{\|}(0)\right|\left|\tau_{\perp}(0)\right|}\right] .
$$

This allows an experimentalist to learn about some of the phase information within the tunnelling matrix.

\subsubsection{Relating $\tau$ to sub-lattice tunnel-coupling}

In SLG, the pseudo-spin represents an electron occupying either sub-lattice $\uparrow \equiv \mathbf{A}$ or $\downarrow \equiv \mathbf{B}$. It is then straight forward to see that $\tau$ represents the tunnel-coupling of sub-lattices between two systems. We can label our pseudo-spin using sub-latices $\left\{\mathbf{A}_{1}, \mathbf{B}_{1}\right\}$ and $\left\{\mathbf{A}_{2}, \mathbf{B}_{2}\right\}$. We then find the coupling between sub-lattices is represented by

$$
\tau=\left(\begin{array}{cc}
\tau_{\mathbf{A}_{1} \mathbf{A}_{2}} & \tau_{\mathbf{A}_{1} \mathbf{B}_{2}} \\
\tau_{\mathbf{B}_{1} \mathbf{A}_{2}} & \tau_{\mathbf{B}_{1} \mathbf{B}_{2}}
\end{array}\right) .
$$

We can relate the coupling between sub-lattices to our previous parametrization $\tau=$ $\frac{1}{\sqrt{2}}\left[\tau_{0} \sigma_{0}+\tau_{x} \sigma_{x}+\tau_{y} \sigma_{y}+\tau_{z} \sigma_{z}\right]$. In this parametrization we find the same tunnelling matrix as

$$
\tau=\frac{1}{\sqrt{2}}\left(\begin{array}{cc}
\tau_{0}+\tau_{z} & \tau_{x}+i \tau_{y} \\
\tau_{x}-i \tau_{y} & \tau_{0}-\tau_{z}
\end{array}\right)
$$


In this form, one can see that if $\tau_{0}, \tau_{x}, \tau_{y}$ and $\tau_{z}$ are all real valued, then $\tau$ is Hermitian.

When $\tau$ is Hermitian, $\left|\tau_{\mathbf{A}_{1} \mathbf{B}_{2}}\right|^{2}=\left|\tau_{\mathbf{B}_{1} \mathbf{A}_{2}}\right|^{2}$, so the probability rate of tunnelling between $\mathbf{A}_{1} \leftrightarrow \mathbf{B}_{2}$ is the same as tunnelling between $\mathbf{B}_{1} \leftrightarrow \mathbf{A}_{2}$. However, if both $\tau_{0} \neq 0$ and $\tau_{z} \neq 0$, one will find that $\left|\tau_{\mathbf{A}_{1} \mathbf{A}_{2}}\right|^{2} \neq\left|\tau_{\mathbf{B}_{1} \mathbf{B}_{2}}\right|^{2}$. In general, this means the probability rate of tunnelling between $\mathbf{A}_{1} \leftrightarrow \mathbf{A}_{2}$ is not the same as tunnelling between $\mathbf{B}_{1} \leftrightarrow \mathbf{B}_{2}$.

Also, if $\tau$ is Hermitian, one can interpret $\vec{\tau}=\left(\tau_{x}, \tau_{y}, \tau_{z}\right)$ as a real valued vector pointing in the direction of a pseudo-spin flip. One can use the relation

$$
i\|\vec{\tau}\| \mathrm{e}^{-i \frac{\pi}{2} \frac{\vec{\tau}}{\|\vec{\tau}\|} \cdot \vec{\sigma}}=\vec{\tau} \cdot \vec{\sigma}
$$

to show that

$$
\tau=\frac{1}{\sqrt{2}}\left[\tau_{0} \sigma_{0}+i\|\vec{\tau}\| \mathrm{e}^{-i \frac{\pi}{2} \| \vec{\tau}} \cdot \vec{\sigma}\right] .
$$

However, this equality does not hold when $\tau$ is not Hermitian, because then $\vec{\tau}$ is not real valued.

When $\tau$ is not Hermitian, there is no restriction on the couplings between the two systems. So all couplings could be independent.

\subsubsection{Alignment of sub-lattices and structure of the barrier}

One can speculate how the alignment of sub-lattices and the barrier itself may affect the components of $\tau$. For example, if the barrier is a vacuum, then the coupling will depend strongly on the wave function overlap between the initial and final tunnelling states. In SLG, the wave functions are determined by the $p_{z}$-orbitals of the sublattices, which implies that the coupling is determined by the separation of the sublattices. For tunnelling to occur, it is reasonable to assume that the coupling requires the wave-functions to overlap between the sub-lattices $\left\{\mathbf{A}_{1}, \mathbf{B}_{1}\right\}$ and $\left\{\mathbf{A}_{2}, \mathbf{B}_{2}\right\}$. In particular [27] shows that the tunnelling matrix element can be expressed in terms of the wave-functions in each system:

$$
\tau_{\sigma \sigma^{\prime}}=\int_{\text {System } 2} \psi_{2, \sigma^{\prime}}^{*}(\mathbf{r}) U_{2}(\mathbf{r}) \psi_{1, \sigma}(\mathbf{r}) \mathrm{d}^{3} r,
$$

where $\sigma$ is represents sub-lattice, and $U_{2}$ is the potential for system 2. From this equation, it is clear how the tunnelling matrix elements will depend on the wavefunction overlap between two sub-lattices. And thus, how the tunnelling matrix elements will depend on the separation distance between the sub-lattices. This means that the coupling between sub-lattices should change when one translates one sublattice relative to the other, as shown in Fig. 5.1.

When one includes a thin barrier, which is typical for a van der Waals heterostructure, the barrier will influence the coupling between sub-lattices. In particular, one can look at the total coupling between the two SLG systems as the sum of possible nearest neighbour couplings through the barrier. One can view this as the sum of possible paths through the barrier, from one sub-lattice to another, as seen in Fig. 


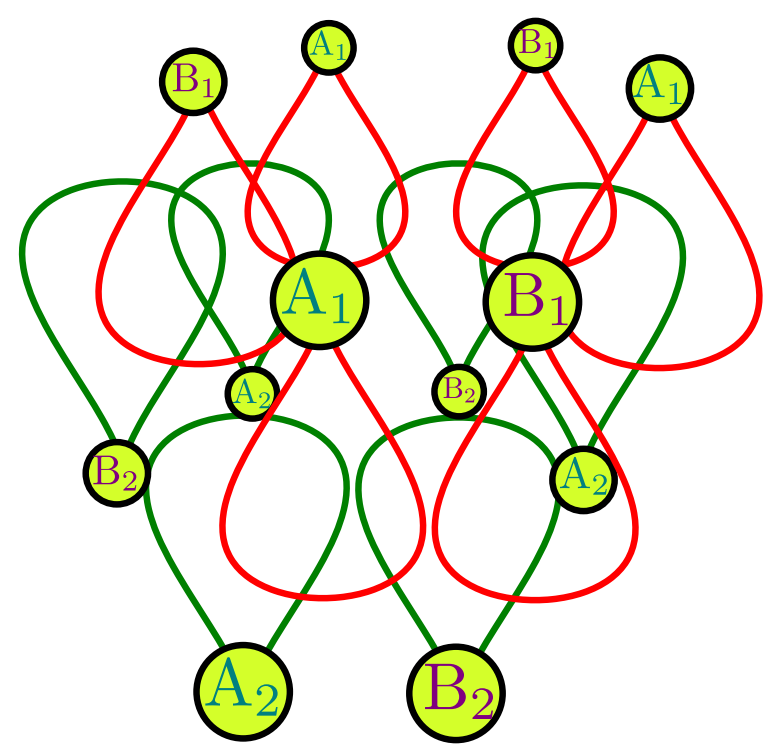

Figure 5.1: Two SLG lattices are separated by a vacuum, with the $p_{z}$-orbitals for each sub-lattice overlapping with the other layer. Since the top layer has been translated relative to the bottom layer, the wave function overlap between the sub-lattices has been altered. This suggests that translating one system relative to the other will change the pseudo-spin coupling between the systems. So that the coupling is strongly determined by the alignment of the sub-lattices between the SLG layers.

5.2. In this view, the barrier could also cause constructive and destructive interference for tunnelling due to phase effects (which also justifies using a complex valued $\tau)$. The implies that the barrier will play a large role in determining the pseudo-spin coupling, with the couplings along the possible tunnelling paths determining $\tau$.

\subsection{Valley polarisation of current between single and bilayer graphene}

In this chapter, we investigate a Van der Waals heterostructure in the form of SLG/barrier/BLG. We focus on the linear magneto-tunnelling conductance for an in-plane magnetic field $\mathbf{B}=B_{\|} \hat{\mathbf{b}}$, and look at how the conductance is different for tunnelling between $\mathbf{K}_{\mathrm{slg}} \leftrightarrow \mathbf{K}_{\mathrm{blg}}$ and $\mathbf{K}_{\mathrm{slg}}^{\prime} \leftrightarrow \mathbf{K}_{\mathrm{blg}}^{\prime}$. We find a situation where the magneto-tunnelling transport creates a valley-filter, where tunnelling can only occur for a single valley. A realised valley-filter would provide control over the valley degree of freedom for electrons in graphene for information processing [43 -45]. 


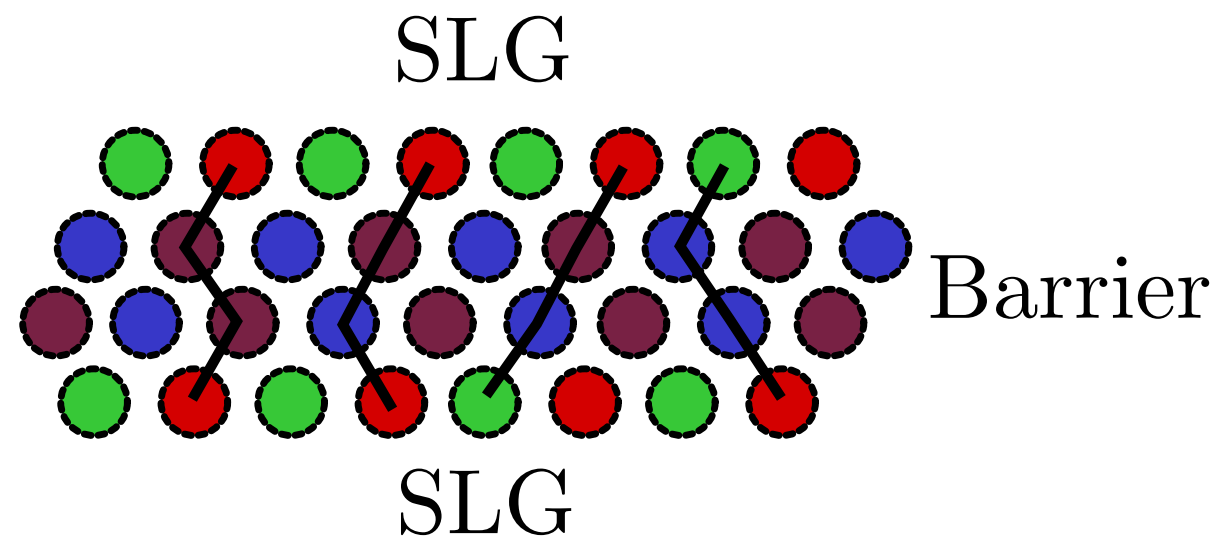

Figure 5.2: Two SLG lattices separated by a tunnelling barrier (in practice, they are 4 layers or more). One can view the couplings between each layer as a sum of nearest neighbour couplings through the barrier. For example, the black lines represent possible paths through the barrier, from one sub-lattice to another. The net coupling due to these paths will determine $\tau$.

\subsubsection{Effective tunnel coupling between single layer and bi- layer graphene}

In this section, we focus on the single particle Hamiltonian for coupled SLG and BLG systems. Since BLG is two stacked layers of SLG, BLG can be described by a $4 \times 4$ matrix. However, at low energies, two eigenstates become inaccessible, previously allowing us to approximate BLG using a $2 \times 2$ matrix through Löwdin partitioning. We now use Löwdin partitioning for both coupled systems, to see how removing the two inaccessible eigenstates affects the coupling between SLG and BLG.

One can write the single particle Hamiltonian in the form of

$$
\mathcal{H}=\left(\begin{array}{cc}
\mathcal{H}^{(\mathrm{slg})} & \mathcal{T} \\
\mathcal{T}^{\dagger} & \mathcal{H}^{(\mathrm{blg})}
\end{array}\right)
$$

Using the sub-lattice representation $\{A, B, A 1, B 1, A 2, B 2\}$, this Hamiltonian is

$$
\mathcal{H}=\left(\begin{array}{cccccc}
0 & v \hbar k_{-} & \tau_{A, A 1} & \tau_{A, B 1} & \tau_{A, A 2} & \tau_{A, B 2} \\
v \hbar k_{+} & 0 & \tau_{B, A 1} & \tau_{B, B 1} & \tau_{B, A 2} & \tau_{B, B 2} \\
\tau_{A, A 1}^{*} & \tau_{B, A 1}^{*} & 0 & v \hbar k_{+} & \gamma_{1} & 0 \\
\tau_{A, B 1}^{*} & \tau_{B, B 1}^{*} & v \hbar k_{-} & 0 & 0 & 0 \\
\tau_{A, A 2}^{*} & \tau_{B, A 2}^{*} & \gamma_{1} & 0 & 0 & v \hbar k_{-} \\
\tau_{A, B 2}^{*} & \tau_{B, B 2}^{*} & 0 & 0 & v \hbar k_{+} & 0
\end{array}\right)
$$


We can then change the basis using the matrix

$$
\Lambda=\left(\begin{array}{cccccc}
1 & 0 & 0 & 0 & 0 & 0 \\
0 & 1 & 0 & 0 & 0 & 0 \\
0 & 0 & 0 & 1 & 0 & 0 \\
0 & 0 & 0 & 0 & 0 & 1 \\
0 & 0 & \frac{1}{\sqrt{2}} & 0 & \frac{1}{\sqrt{2}} & 0 \\
0 & 0 & \frac{1}{\sqrt{2}} & 0 & -\frac{1}{\sqrt{2}} & 0
\end{array}\right) .
$$

This puts one in the position to use Löwdin partitioning to project on to the $\{A, B, B 1, B 2\}$ partition of the Hamiltonian, giving an approximate $4 \times 4$ Hamiltonian. To zeroth order, one finds

$$
\mathcal{H}^{(0)}=0 .
$$

The first order contribution consists of the SLG Hamiltonian, and couplings between the SLG and BLG states

$$
\mathcal{H}^{(1)}=\left(\begin{array}{cccc}
0 & v \hbar k_{-} & \tau_{A, B 1} & \tau_{A, B 2} \\
v \hbar k_{+} & 0 & \tau_{B, B 1} & \tau_{B, B 2} \\
\tau_{A, B 1}^{*} & \tau_{B, B 1}^{*} & 0 & 0 \\
\tau_{A, B 2}^{*} & \tau_{B, B 2}^{*} & 0 & 0
\end{array}\right)
$$

To second order, one finds the Hamiltonian

$\mathcal{H}^{(2)}=-\frac{1}{\gamma_{1}}\left(\begin{array}{cccc}\tau_{A, A 1} \tau_{A, A 2}^{*}+\tau_{A, A 1}^{*} \tau_{A, A 2} & \tau_{A, A 1} \tau_{B, A 2}^{*}+\tau_{A, A 2} \tau_{B, A 1}^{*} & v \hbar k_{-} \tau_{A, A 2} & v \hbar k_{+} \tau_{A, A 1} \\ \tau_{B, A 1} \tau_{A, A 2}^{*}+\tau_{A, A 1}^{*} \tau_{B, A 2} & \tau_{B, A 1} \tau_{B, A 2}^{*}+\tau_{B, A 1}^{*} \tau_{B, A 2} & v \hbar k_{-} \tau_{B, A 2} & v \hbar k_{+} \tau_{B, A 1} \\ v \hbar k_{+} \tau_{A, A 2}^{*} & v \hbar k_{+} \tau_{B, A 2}^{*} & 0 & (v \hbar)^{2} k_{+}^{2} \\ v \hbar k_{-} \tau_{A, A 1}^{*} & v \hbar k_{-} \tau_{B, A 1}^{*} & (v \hbar)^{2} k_{-}^{2} & 0\end{array}\right)$.

One can see the form of the familiar BLG Hamiltonian. However, one can see extra terms due to the couplings between SLG and BLG. In the case that $\frac{v \hbar^{2}}{\gamma_{1}} \ll 1$ and each term of $\tau$ is small, we find that these terms can be neglected, and only the BLG Hamiltonian will remain

$$
\mathcal{H}^{(2)} \approx\left(\begin{array}{cccc}
0 & 0 & 0 & 0 \\
0 & 0 & 0 & 0 \\
0 & 0 & 0 & -\frac{(v \hbar)^{2}}{\gamma_{1}} k_{+}^{2} \\
0 & 0 & -\frac{(v \hbar)^{2}}{\gamma_{1}} k_{-}^{2} & 0
\end{array}\right)
$$

If one lets $v^{2} / \gamma_{1}=1 / 2 M$, one obtains the simplified result

$$
\mathcal{H} \approx\left(\begin{array}{cccc}
0 & v \hbar k_{-} & \tau_{A, B 1} & \tau_{A, B 2} \\
v \hbar k_{+} & 0 & \tau_{B, B 1} & \tau_{B, B 2} \\
\tau_{A, B 1}^{*} & \tau_{B, B 1}^{*} & 0 & -\frac{\hbar^{2}}{2 M} k_{+}^{2} \\
\tau_{A, B 2}^{*} & \tau_{B, B 2}^{*} & -\frac{\hbar^{2}}{2 M} k_{-}^{2} & 0
\end{array}\right)
$$

where the tunnelling matrix is straightforward. Here, we have justified that we can ignore the coupling to the $A 1$ and $A 2$ sub-lattices. It follows that we can use Eq. (5.21) to calculate the linear magneto-tunnelling conductance between the SLG and BLG graphene systems. 


\subsubsection{Generation of a valley polarisation}

To calculate the total linear magneto-tunnelling conductance for $\mathbf{B}=B_{\|} \hat{\mathbf{y}}$, one can use Eq. 4.8. However, in this section we find it useful to label the linear magnetotunnelling conductance for each valley

$$
\begin{aligned}
& G^{(\gamma)}=\frac{g_{\mathrm{s}} e^{2}}{\hbar} 2 \pi A \rho_{\mathrm{F}}^{(1)} \rho_{\mathrm{F}}^{(2)}\left[\left|\Gamma_{\mathrm{u}}^{(\gamma)}\right|^{2}+\left|\Gamma_{\mathrm{l}}^{(\gamma)}\right|^{2}\right] \\
& \times \frac{\Theta\left(|\mathbf{Q}|-\left|k_{\mathrm{F}}^{(1)}-k_{\mathrm{F}}^{(2)}\right|\right) \Theta\left(k_{\mathrm{F}}^{(1)}+k_{\mathrm{F}}^{(2)}-|\mathrm{Q}|\right)}{\sqrt{\left[\left(k_{\mathrm{F}}^{(1)}+k_{\mathrm{F}}^{(2)}\right)^{2}-Q^{2}\right]\left[Q^{2}-\left(k_{\mathrm{F}}^{(1)}-k_{\mathrm{F}}^{(2)}\right)^{2}\right]}} .
\end{aligned}
$$

To quantify the how much current is passing through one valley relative to the other, we define the valley polarisation

$$
\chi=\frac{G^{\mathbf{K}}-G^{\mathbf{K}^{\prime}}}{G^{\mathbf{K}}+G^{\mathbf{K}^{\prime}}} .
$$

When $\chi= \pm 1$ one will find $G(0)=G^{\mathbf{K}}$ or $G(0)=G^{\mathbf{K}^{\prime}}$. Using Eq. (5.22), the valley polarisation can be written as

$$
\chi=\frac{\left[\left|\Gamma_{\mathrm{u}}^{\mathbf{K}}\right|^{2}+\left|\Gamma_{1}^{\mathbf{K}}\right|^{2}\right]-\left[\left|\Gamma_{\mathrm{u}}^{\mathbf{K}^{\prime}}\right|^{2}+\left|\Gamma_{1}^{\mathbf{K}^{\prime}}\right|^{2}\right]}{\left|\Gamma_{\mathrm{u}}^{\mathbf{K}}\right|^{2}+\left|\Gamma_{1}^{\mathbf{K}}\right|^{2}+\left|\Gamma_{\mathrm{u}}^{\mathbf{K}^{\prime}}\right|^{2}+\left|\Gamma_{1}^{\mathbf{K}^{\prime}}\right|^{2}} .
$$

Following work in Sec. 4.2, one can write the pseudo-spin eigenstates using the rotation $\mathcal{U}(\theta)=\exp \left(-i \sigma_{z} \theta / 2\right)$ and pseudo-spin along the x-axis $|\rightarrow\rangle$ :

$$
\begin{aligned}
\left|+_{\mathrm{F}}^{(\mathrm{slg})} \mathbf{K}, \boldsymbol{\Pi}_{\mathrm{u}}^{(\mathrm{slg})}\right\rangle & =\mathcal{U}\left(\theta_{\boldsymbol{\Pi}_{\mathrm{u}}^{(\mathrm{slg})}}\right)|\rightarrow\rangle, \\
\left|+_{\mathrm{F}}^{(\mathrm{slg})} \mathbf{K}^{\prime}, \boldsymbol{\Pi}_{\mathrm{u}}^{(\mathrm{slg})}\right\rangle & =\mathcal{U}\left(\pi-\theta_{\left.\mathbf{\Pi}_{\mathrm{u}}^{(\mathrm{slg})}\right)}\right)|\rightarrow\rangle, \\
\left|+_{\mathrm{F}}^{(\mathrm{blg})} \mathbf{K}, \boldsymbol{\Pi}_{\mathrm{u}}^{(\mathrm{blg})}\right\rangle & =\mathcal{U}\left(-2 \theta_{\mathbf{\Pi}_{\mathrm{u}}^{(\mathrm{blg})}}\right)|\leftarrow\rangle, \\
\left|+_{\mathrm{F}}^{(\mathrm{blg})} \mathbf{K}^{\prime}, \boldsymbol{\Pi}_{\mathrm{u}}^{(\mathrm{blg})}\right\rangle & =\mathcal{U}\left(2 \theta_{\boldsymbol{\Pi}_{\mathrm{u}}^{(\mathrm{blg})}}\right)|\leftarrow\rangle .
\end{aligned}
$$

This allows one to write the momentum-resolved tunnelling matrix elements as

$$
\begin{aligned}
& \left|\Gamma_{\mathrm{u}}^{\mathbf{K}}\right|^{2}=\left|\left\langle\leftarrow\left|\mathcal{U}\left(2 \theta_{\boldsymbol{\Pi}_{\mathrm{u}}^{(\mathrm{blg})}}\right) \tau \mathcal{U}\left(\theta_{\boldsymbol{\Pi}_{\mathrm{u}}^{(\mathrm{slg})}}\right)\right| \rightarrow\right\rangle\right|^{2}, \\
& \left|\Gamma_{1}^{\mathbf{K}}\right|^{2}=\left|\left\langle\leftarrow\left|\mathcal{U}\left(2 \theta_{\Pi_{\mathrm{u}}^{(\mathrm{blg})}}\right) \sigma_{x} \tau \sigma_{x} \mathcal{U}\left(\theta_{\boldsymbol{\Pi}_{\mathrm{u}}^{(\mathrm{slg})}}\right)\right| \rightarrow\right\rangle\right|^{2}, \\
& \left|\Gamma_{\mathrm{u}}^{\mathbf{K}^{\prime}}\right|^{2}=\left|\left\langle\leftarrow\left|\mathcal{U}\left(2 \theta_{\boldsymbol{\Pi}_{\mathrm{u}}^{(\mathrm{blg})}}\right) \sigma_{x} \tau \sigma_{y} \mathcal{U}\left(\theta_{\boldsymbol{\Pi}_{\mathrm{u}}^{(\mathrm{slg})}}\right)\right| \rightarrow\right\rangle\right|^{2}, \\
& \left|\Gamma_{1}^{\mathbf{K}^{\prime}}\right|^{2}=\left|\left\langle\leftarrow\left|\mathcal{U}\left(2 \theta_{\boldsymbol{\Pi}_{\mathrm{u}}^{(\mathrm{blg})}}\right) \sigma_{z} \tau \mathcal{U}\left(\theta_{\boldsymbol{\Pi}_{\mathrm{u}}^{(\mathrm{slg})}}\right)\right| \rightarrow\right\rangle\right|^{2} .
\end{aligned}
$$

It is worth noting that the pseudo-spin eigenstates in SLG transform from the $\mathbf{K} \rightarrow \mathbf{K}^{\prime}$ valley as $\theta \rightarrow \pi-\theta$, while in BLG they transform as $2 \theta \rightarrow-2 \theta$. These transformations have been represented with Pauli matrices using the relation $R_{\nu}(\pi)=i \sigma_{\nu}$ 


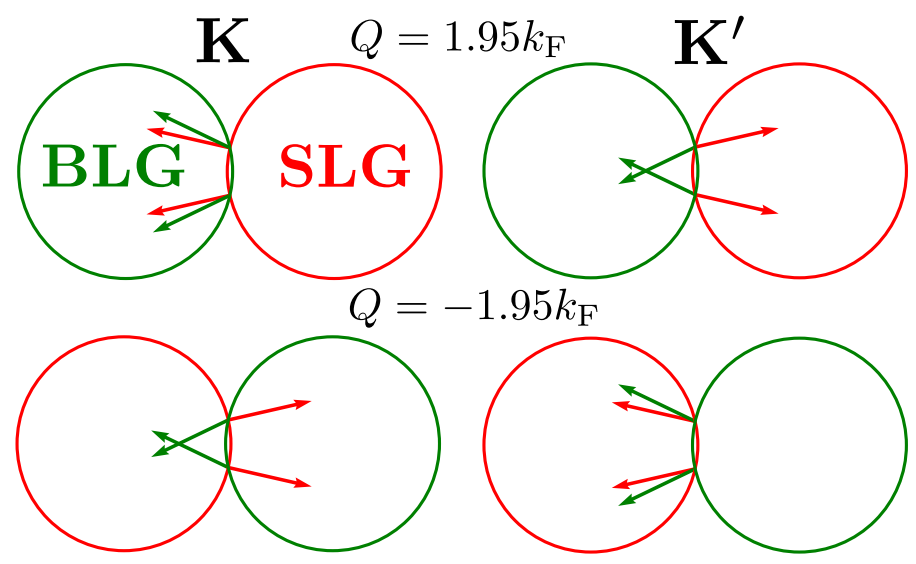

Figure 5.3: When $Q= \pm 2 k_{\mathrm{F}}$, the Fermi circles kiss, and the pseudo-spin lies along the $x$-axis. When $Q \approx 2 k_{\mathrm{F}}$, we see that the $\mathbf{K}$ valley pseudo-spins will align at $2 k_{\mathrm{F}}$ while the $\mathbf{K}^{\prime}$ valley pseudo-spins will be orthogonal. This allows tunnelling for only one valley at the kissing point.

(which is discussed in work from Sec. 4.2). The difference in how the SLG and BLG eigenstates transform between their respective valleys is the source of valley polarization effects.

For a general tunnelling matrix, the valley polarisation at $Q= \pm\left[k_{\mathrm{F}}^{(\mathrm{slg})}+k_{\mathrm{F}}^{(\mathrm{blg})}\right]$, the kissing point between the two Fermi circles, can be calculated by inputting $\theta_{\boldsymbol{\Pi}_{\mathrm{u}}^{(\mathrm{slg})}}=0$ and $\theta_{\boldsymbol{\Pi}_{\mathrm{u}}^{(\mathrm{blg})}}=\pi$, or $\theta_{\boldsymbol{\Pi}_{\mathrm{u}}^{(\mathrm{slg})}}=\pi$ and $\theta_{\boldsymbol{\Pi}_{\mathrm{u}}^{(\mathrm{blg})}}=0$. One can then show that the valley polarisation at $Q= \pm\left[k_{\mathrm{F}}^{(\mathrm{slg})}+k_{\mathrm{F}}^{(\mathrm{blg})}\right]$ is

$$
\chi\left(\mathbf{Q}= \pm\left[k_{\mathrm{F}}^{(\mathrm{slg})}+k_{\mathrm{F}}^{(\mathrm{blg})}\right] \hat{\mathbf{x}}\right)= \pm \frac{\left|\tau_{0}-\tau_{x}\right|^{2}-\left|\tau_{y}+i \tau_{z}\right|^{2}}{\left|\tau_{0}-\tau_{x}\right|^{2}+\left|\tau_{y}+i \tau_{z}\right|^{2}}
$$

It is clear that there is a valley filter at the kissing point when $\tau=\left[\tau_{0} \sigma_{0}+\tau_{x} \sigma_{x}\right] / \sqrt{2}$ or $\tau=\left[\tau_{y} \sigma_{y}+\tau_{z} \sigma_{z}\right] / \sqrt{2}$. This valley filter is a result of the pseudo-spin overlap at the $\mathrm{u} / \mathrm{l}$ intersections shown in Fig. 5.3. The pseudo-spins are shown to be parallel in one valley, and orthogonal in the other.

When the pseudo-spin coupling is $\tau=\left[\tau_{0} \sigma_{0}+\tau_{x} \sigma_{x}\right] / \sqrt{2}$, the magneto-tunnelling conductance can be written as

$$
\begin{aligned}
G^{(\mathrm{slg}) \leftrightarrow(\mathrm{blg})}(0)=G^{\mathbf{K}}+G^{\mathbf{K}^{\prime}} & =\frac{e^{2} A}{\pi \hbar} \frac{\left(k_{\mathrm{F}}^{(\mathrm{slg})} / v \hbar\right)\left(m / \hbar^{2}\right)}{\sqrt{\left[\left(k_{\mathrm{F}}^{(\mathrm{blg})}+k_{\mathrm{F}}^{(\mathrm{slg})}\right)^{2}-Q^{2}\right]\left[Q^{2}-\left(k_{\mathrm{F}}^{(\mathrm{blg})}-k_{\mathrm{F}}^{(\mathrm{slg})}\right)^{2}\right]}} \\
& \times\left[2\left|\tau_{0}+\tau_{x}\right|^{2}-\Re\left\{\tau_{0} \tau_{x}^{*}\right\} \frac{\left(Q^{2}+\left(k_{\mathrm{F}}^{(\mathrm{blg})}\right)^{2}-\left(k_{\mathrm{F}}^{(\mathrm{slg})}\right)^{2}\right)^{2}}{Q^{2}\left(k_{\mathrm{F}}^{(\mathrm{blg})}\right)^{2}}\right] .
\end{aligned}
$$

At the kissing point $Q= \pm\left[k_{\mathrm{F}}^{(\mathrm{slg})}+k_{\mathrm{F}}^{(\mathrm{blg})}\right]$, there is a divergence in the magnetotunnelling conductance. In practice, this divergence will become a resonance that is 


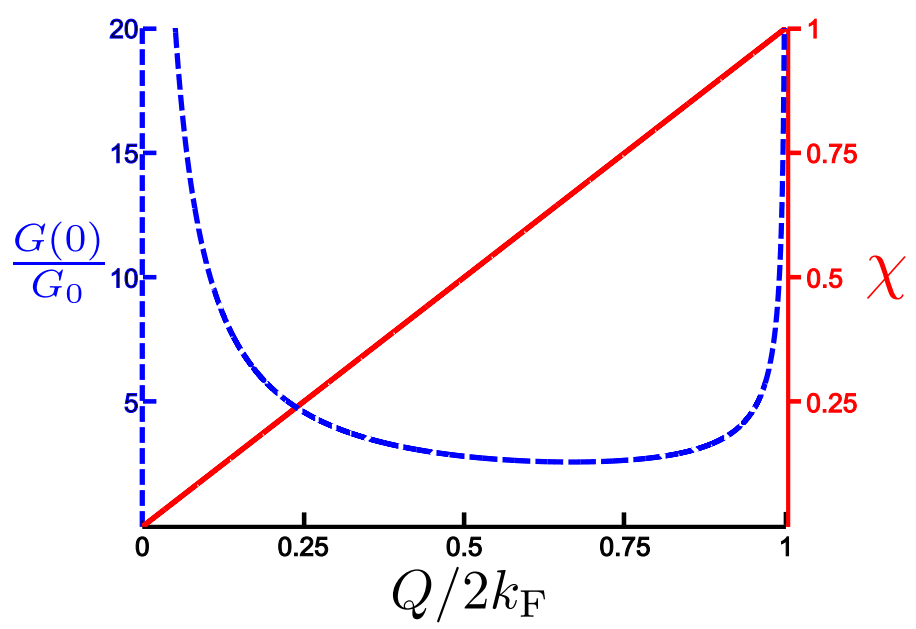

Figure 5.4: The valley polarisation $\chi$ (red) is compared with the magneto-tunnelling conductance $G(0)$ (blue). The magneto-tunnelling conductance is in units of $G_{0}=$ $e^{2} M A\left|\tau_{0}\right|^{2} /\left(2 \pi \hbar^{4} v k_{\mathrm{F}}\right)$, where both systems have equal electron densities and pseudospin is conserved in the tunnelling process.

a function of the electron life-time, emphasising that $G^{(\mathrm{slg}) \leftrightarrow(\mathrm{blg})}(0) \neq 0$ at the kissing point. When $\tau=\tau_{0} \sigma_{0} / \sqrt{2}$, one finds

$$
\chi=\sin ^{2}\left(\frac{\theta_{\boldsymbol{\Pi}_{\mathrm{u}}^{(\mathrm{slg})}}+2 \theta_{\boldsymbol{\Pi}_{\mathrm{u}}^{(\mathrm{blg})}}}{2}\right)-\cos ^{2}\left(\frac{\theta_{\boldsymbol{\Pi}_{\mathrm{u}}^{(\mathrm{slg})}}+2 \theta_{\boldsymbol{\Pi}_{\mathrm{u}}^{(\mathrm{blg})}}}{2}\right) .
$$

In the case that $k_{\mathrm{F}}^{(\mathrm{slg})}=k_{\mathrm{F}}^{(\mathrm{blg})}$, the valley polarisation can be put in the simple algebraic form

$$
\chi=\frac{Q}{2 k_{\mathrm{F}}} \text {. }
$$

Which states that there is a valley filter at the kissing points $Q= \pm 2 k_{\mathrm{F}}$. This can be seen in Fig. 5.4, where it is compared with the magneto-tunnelling conductance for pseudo-spin conserved tunnelling

$$
\frac{G(0)}{G_{0}}=\frac{4 k_{\mathrm{F}}^{2}}{Q \sqrt{4 k_{\mathrm{F}}^{2}-Q^{2}}}
$$

where $G_{0}=e^{2} M A\left|\tau_{0}\right|^{2} /\left(2 \pi \hbar^{4} v k_{\mathrm{F}}\right)$. Again, we see a resonance in $G(0)$ at $Q=2 k_{\mathrm{F}}$, resulting in a finite current when $\chi= \pm 1$.

Using Eq. (5.29), the maximum possible valley polarization is then limited by the maximum magnetic field

$$
\chi_{\max } \leq \min \left\{1, \frac{e}{\hbar} \frac{B^{(\max )} d}{\sqrt{4 \pi n}} \equiv 0.05 \times \frac{B^{(\max )}[\mathrm{T}] d[\mathrm{~nm}]}{\sqrt{n\left[10^{10} \mathrm{~cm}^{-2}\right]}}\right\} .
$$

Methods to generate a valley polarized current require breaking inversion/timereversal symmetry, methods suggested in other works involve nanostructuring of 
a graphene sample [44, 57], coupling to electromagnetic fields [58 66], application of mechanical strain [61 63, 66 68], or the presence of defects 69]. However, the method proposed in this work is unique, it requires an applied magnetic field and an appropriate pseudo-spin coupling between the single layer and bilayer graphene systems to generate a valley polarized current.

\subsection{Effect of an in-plane electric field on magneto- tunneling current}

In this section, we look at the effect of a constant electric field applied perpendicular and parallel to the magnetic field in two SLG layers, and investigate the effect on the magneto-tunnelling conductance between them.

It can be shown that when an electric field is applied in the $x y$ plane, we should have a Hamiltonian of the form [7]:

$$
\mathcal{H}_{ \pm \mathbf{K}}^{\mathrm{slg}}(\mathbf{B}, \mathcal{E})=v\left(\Pi_{x} \sigma_{x}+\Pi_{y} \sigma_{y} \pm\left(F_{x} \Pi_{y}-F_{y} \Pi_{x}\right) \sigma_{z}\right)
$$

where $\mathcal{E}$ is an in-plane electric field, and $F_{i}$ is an energy such that $F_{i} \propto \mathcal{E}_{i}$. We calculate the magneto-tunnelling conductance following the same methods in chapter 3

\subsubsection{Electric field perpendicular to the in-plane magnetic field}

One can look at an electric field $\mathcal{E}_{\perp} \hat{\mathbf{x}}$ perpendicular to the in-plane magnetic field $B_{\|} \hat{\mathbf{y}}$ as shown in Fig. 5.5. The Hamiltonian is

$$
\mathcal{H}_{ \pm \mathbf{K}}^{\mathrm{slg}}=v\left(\Pi_{x} \sigma_{x}+\Pi_{y} \sigma_{y} \pm F_{\perp} \Pi_{y} \sigma_{z}\right)
$$

The energies are

$$
\varepsilon_{\gamma, \sigma, \mathbf{k}}=\sigma v \sqrt{\Pi_{x}^{2}+\left(1+F_{\perp}^{2}\right) \Pi_{y}^{2}} .
$$

The pseudo-spin eigenstates are

$|\sigma\rangle_{\mathbf{K}, \boldsymbol{\Pi}(\mathbf{k}, \mathbf{B})}=\frac{1}{\sqrt{2}}\left(\begin{array}{l}\mathrm{e}^{-i \frac{\theta_{\Pi}}{2}} \sqrt{\frac{\frac{\varepsilon_{\mathbf{k}, \sigma}+v F_{\perp} \Pi_{y}}{\varepsilon_{\mathbf{k}, \sigma}}}{\sigma \mathrm{e}^{i \frac{\theta_{\Pi}}{2}}}} \sqrt{\frac{\varepsilon_{\mathbf{k}, \sigma}-v F_{\perp} \Pi_{y}}{\varepsilon_{\mathbf{k}, \sigma}}}\end{array}\right),|\sigma\rangle_{\mathbf{K}^{\prime}, \boldsymbol{\Pi}(\mathbf{k}, \mathbf{B})}=\frac{1}{\sqrt{2}}\left(\begin{array}{l}\mathrm{e}^{-i \frac{\pi-\theta_{\Pi}}{2}} \sqrt{\frac{\varepsilon_{\mathbf{k}, \sigma}-v F_{\perp} \Pi_{y}}{\varepsilon_{\mathbf{k}, \sigma}}} \\ \sigma \mathrm{e}^{i \frac{\pi-\theta_{\boldsymbol{\Pi}}}{2}} \sqrt{\frac{\varepsilon_{\mathbf{k}, \sigma}+v F_{\perp} \Pi_{y}}{\varepsilon_{\mathbf{k}, \sigma}}}\end{array}\right)$.

One can evaluate Eq. (4.4) using a substitution to obtain the linear magnetotunnelling conductance. Eq. (5.34) shows that the momentum values at the Fermi energy parametrize an ellipse rather than a circle. Now, the linear magneto-tunnelling conductance is determined where the two Fermi ellipses intersect $\mathbf{k}_{\mathrm{u} / \mathrm{l}}$.

One will find that since the Fermi momentum does not have a constant magnitude, one has to express the the linear magneto-tunnelling conductance using the Fermi 


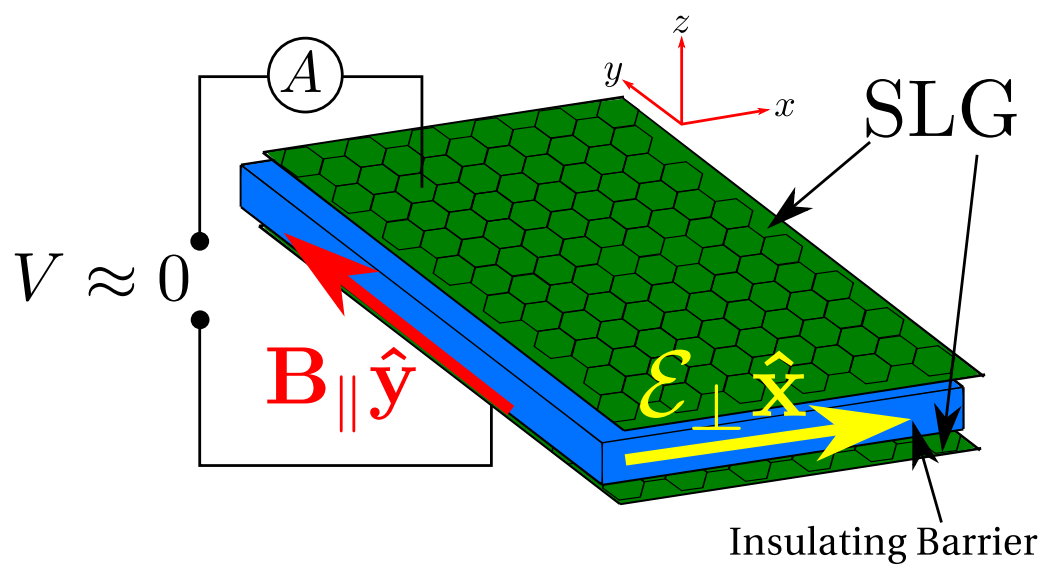

Figure 5.5: Two layers of SLG separated by a barrier. A constant magnetic field $\mathbf{B}=B_{\|} \hat{\mathbf{y}}$ and electric field $\mathcal{E}=\mathcal{E}_{\perp} \hat{\mathbf{x}}$ are applied across the systems and barrier. The red arrow represents the magnetic field, and the yellow arrow represents the electric field.

energy

$$
\begin{aligned}
G^{(\mathrm{slg})}(0) & =\frac{g_{\mathrm{s}} \mathrm{e}^{2} A}{\pi \hbar \sqrt{1+F_{\perp}^{2}}} \frac{\varepsilon_{\mathrm{F}}^{(1)} \varepsilon_{\mathrm{F}}^{(2)}}{(v \hbar)^{2} \sqrt{\left[\left(\varepsilon_{\mathrm{F}}^{(1)}+\varepsilon_{\mathrm{F}}^{(2)}\right)^{2}-\hbar^{2} v^{2} Q^{2}\right]\left[\hbar^{2} v^{2} Q^{2}-\left(\varepsilon_{\mathrm{F}}^{(1)}-\varepsilon_{\mathrm{F}}^{(2)}\right)^{2}\right]}} \\
& \times \Theta\left(\hbar v|\mathbf{Q}|-\left|\varepsilon_{\mathrm{F}}^{(1)}-\varepsilon_{\mathrm{F}}^{(2)}\right|\right) \Theta\left(\varepsilon_{\mathrm{F}}^{(1)}+\varepsilon_{\mathrm{F}}^{(2)}-\hbar v|\mathbf{Q}|\right) \sum_{\gamma}\left[\left|\Gamma_{\mathrm{u}}^{(\gamma)}\right|^{2}+\left|\Gamma_{\mathrm{l}}^{(\gamma)}\right|^{2}\right]
\end{aligned}
$$

The tunnelling matrix elements at the intersecting Fermi ellipses are

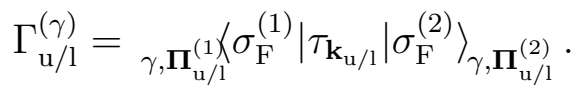

In the case where $\varepsilon_{\mathrm{F}}^{(1)}=\varepsilon_{\mathrm{F}}^{(2)}$ and $\tau_{\mathbf{k}} \equiv \frac{1}{\sqrt{2}} \tau_{0} \sigma_{0}$

$$
G_{\perp}^{(\mathrm{slg})}(0) \equiv G_{n \leftrightarrow n}^{(\mathrm{slg})}(0)=\frac{g_{\mathrm{s}} \mathrm{e}^{2} A\left|\tau_{0}\right|^{2}}{(v \hbar)^{2} \pi \hbar \sqrt{1+F_{\perp}^{2}}} \frac{\sqrt{4\left(\frac{\varepsilon_{F}}{v \hbar}\right)^{2}-Q^{2}}}{Q} .
$$

\subsubsection{Electric field parallel to the in-plane magnetic field}

One can now investigate using the electric field $\mathcal{E}_{\|} \hat{\mathbf{y}}$ parallel to the magnetic field $B_{\| \hat{\mathbf{y}}}$, as shown in Fig. 5.6. The Hamiltonian is

$$
\mathcal{H}_{ \pm \mathbf{K}}^{\mathrm{slg}}=v\left(\Pi_{x} \sigma_{x}+\Pi_{y} \sigma_{y} \mp F_{\|} \Pi_{x} \sigma_{z}\right)
$$

The energies are

$$
\varepsilon_{\gamma, \sigma, \mathbf{k}}=\sigma v \sqrt{\Pi_{x}^{2}+\left(1+F_{\|}^{2}\right) \Pi_{x}^{2}}
$$




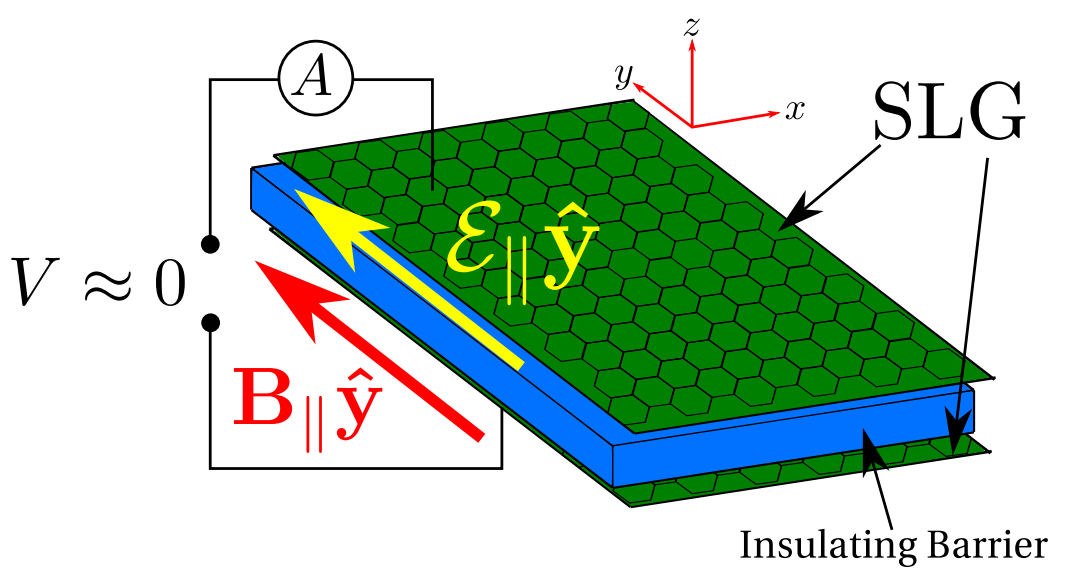

Figure 5.6: Two layers of SLG separated by a barrier. A constant magnetic field $\mathbf{B}=B_{\|} \hat{\mathbf{y}}$ and electric field $\mathcal{E}=\mathcal{E}_{\|} \hat{\mathbf{y}}$ are applied across the systems and barrier. The red arrow represents the magnetic field, and the yellow arrow represents the electric field.

The pseudo-spin eigenstates are

$|\sigma\rangle_{\mathbf{K}, \boldsymbol{\Pi}(\mathbf{k}, \mathbf{B})}=\frac{1}{\sqrt{2}}\left(\begin{array}{l}\mathrm{e}^{-i \frac{\theta_{\Pi}}{2}} \sqrt{\frac{\varepsilon_{\mathbf{k}, \sigma}-v F_{\|} \Pi_{x}}{\varepsilon_{\mathbf{k}, \sigma}}} \\ \sigma \mathrm{e}^{i \frac{\theta_{\Pi}}{2}} \sqrt{\frac{\varepsilon_{\mathbf{k}, \sigma}+v F_{\|} \Pi_{x}}{\varepsilon_{\mathbf{k}, \sigma}}}\end{array}\right),|\sigma\rangle_{\mathbf{K}^{\prime}, \boldsymbol{\Pi}(\mathbf{k}, \mathbf{B})}=\frac{1}{\sqrt{2}}\left(\begin{array}{l}\mathrm{e}^{-i \frac{\pi-\theta_{\Pi}}{2}} \sqrt{\frac{\varepsilon_{\mathbf{k}, \sigma}+v F_{\|} \Pi_{x}}{\varepsilon_{\mathbf{k}, \sigma}}} \\ \sigma \mathrm{e}^{i \frac{\pi-\theta_{\Pi}}{2}} \sqrt{\frac{\varepsilon_{\mathbf{k}, \sigma}-v F_{\|} \Pi_{x}}{\varepsilon_{\mathbf{k}, \sigma}}}\end{array}\right)$.

One can evaluate Eq. (4.4) using a substitution to obtain the magneto-tunnelling conductance. Eq. 5.40 shows that the momentum values at the Fermi energy parametrize an ellipse rather than a circle. Again, the magneto-tunnelling conductance is determined where the two Fermi ellipses intersect $\mathbf{k}_{\mathrm{u} / \mathrm{l}}$.

One will find

$$
\begin{array}{r}
G^{(\mathrm{slg})}(0)=\frac{\frac{g_{\mathrm{s}} \mathrm{e}^{2} A}{\pi \hbar \sqrt{1+F_{\perp}^{2}}} \varepsilon_{\mathrm{F}}^{(1)} \varepsilon_{\mathrm{F}}^{(2)} \sum_{\gamma}\left[\left|\Gamma_{\mathrm{u}}^{(\gamma)}\right|^{2}+\left|\Gamma_{\mathrm{l}}^{(\gamma)}\right|^{2}\right]}{(v \hbar)^{2} \sqrt{\left[\left(\varepsilon_{\mathrm{F}}^{(1)}+\varepsilon_{\mathrm{F}}^{(2)}\right)^{2}-\hbar^{2} v^{2}\left[1+F_{\|}^{2}\right] Q^{2}\right]\left[\hbar^{2} v^{2}\left[1+F_{\|}^{2}\right] Q^{2}-\left(\varepsilon_{\mathrm{F}}^{(1)}-\varepsilon_{\mathrm{F}}^{(2)}\right)^{2}\right]}} . \\
\times \Theta\left(\hbar v|\mathbf{Q}|\left[1+F_{\|}^{2}\right]-\left|\varepsilon_{\mathrm{F}}^{(1)}-\varepsilon_{\mathrm{F}}^{(2)}\right|\right) \Theta\left(\varepsilon_{\mathrm{F}}^{(1)}+\varepsilon_{\mathrm{F}}^{(2)}-\hbar v|\mathbf{Q}|\left[1+F_{\|}^{2}\right]\right)
\end{array}
$$

The tunnelling matrix elements at the intersecting Fermi ellipses are

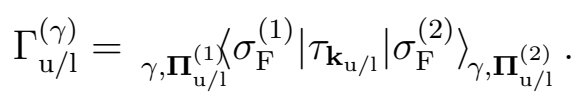

In the case where $\varepsilon_{\mathrm{F}}^{(1)}=\varepsilon_{\mathrm{F}}^{(2)}$ and $\tau_{\mathbf{k}} \equiv \frac{1}{\sqrt{2}} \tau_{0} \sigma_{0}$

$$
G_{\|}^{(\mathrm{slg})}(0) \equiv G_{n \leftrightarrow n}^{(\mathrm{slg})}(0)=\frac{g_{\mathrm{s}} \mathrm{e}^{2} A\left|\tau_{0}\right|^{2}}{(v \hbar)^{2} \pi \hbar \sqrt{1+F_{\|}^{2}}} \frac{\sqrt{4\left(\frac{\varepsilon_{F}}{v \hbar}\right)^{2}-\left[1+F_{\|}^{2}\right] Q^{2}}}{Q \sqrt{1+F_{\|}^{2}}} .
$$




\subsubsection{Noticeable effects of an in-plane electric field}

To see the effects of an in-plane electric field in the magneto-tunnelling conductance in presence of an in-plane magnetic field, one has to first formulate the Fermi energy in terms of the electron density in both layers. The electron density for SLG in an applied electric field is calculated to be

$$
n=\frac{\varepsilon_{\mathrm{F}}^{2}}{\pi v^{2} \hbar^{2}} \frac{1}{\sqrt{1+F_{q}^{2}}},
$$

where $q=\perp, \|$. The density of states at the Fermi energy can now be calculated in terms of the density

$$
\rho_{F}=\frac{\sqrt{n}}{2 v \hbar \sqrt{\pi} \sqrt{\sqrt{1+F_{q}^{2}}}} .
$$

We then choose to define

$$
G_{0}=\frac{2 \pi \mathrm{e}^{2}}{\hbar} \rho_{F}^{2} A \operatorname{Tr}\left\{\tau^{\dagger} \tau\right\} \frac{1}{n \pi} .
$$

Eq. (5.47) is consistent with Eq. (3.27) when $F_{q}=0$, however, it is written in terms of density $n$ rather than Fermi wave number $k_{\mathrm{F}}$. We choose this formulation because when an electric field is applied $k_{\mathrm{F}} \equiv\left\|\mathbf{k}_{\mathrm{F}}\right\|$ is directionally dependent, however, $n$ still only depends on the Fermi energy.

We can now see a renormalized $Q$, moving the divergence in $G_{n \leftrightarrow n}^{(\mathrm{slg})}(0)$ for the $F_{\|}$ term

$$
\frac{G_{\|}^{(\operatorname{slg})}(0)}{G_{0}}=\frac{\sqrt{4 n \pi-\left[Q \sqrt{\sqrt{1+F_{\|}^{2}}}\right]^{2}}}{Q \sqrt{\sqrt{1+F_{\|}^{2}}}},
$$

and for the $F_{\perp}$ term

$$
\frac{G_{\perp}^{(\operatorname{slg})}(0)}{G_{0}}=\frac{\sqrt{4 n \pi-\left[\frac{Q}{\sqrt{\sqrt{1+F_{\perp}^{2}}}}\right]^{2}}}{\frac{Q}{\sqrt{\sqrt{1+F_{\perp}^{2}}}}}
$$

Each equation above can be explained from each Fermi surface being stretched/contracted into a Fermi ellipse from the applied electric field. This changes the where the Fermi ellipses cease to intersect, which is accounted for in the re-normalization of $Q$. In particular, $G_{\|}^{(\mathrm{slg})}(0)=0$ when $Q=\sqrt{\frac{4 n \pi}{\sqrt{1+F_{\|}^{2}}}}$ and $G_{\perp}^{(\mathrm{slg})}(0)=0$ when $Q=\sqrt{4 n \pi \sqrt{1+F_{\perp}^{2}}}$. For an in-plane electric field, when $Q \rightarrow 0$ one will also find

$$
\frac{G_{\|}^{(\mathrm{slg})}(0)}{G_{0}} \rightarrow \frac{1}{Q} \sqrt{\frac{4 n \pi}{\sqrt{1+F_{\|}^{2}}}}
$$


and

$$
\frac{G_{\perp}^{(\mathrm{slg})}(0)}{G_{0}} \rightarrow \frac{1}{Q} \sqrt{4 n \pi \sqrt{1+F_{\perp}^{2}}}
$$

which also allows one to observe the effect of an in-plane electric field.

\subsection{Measuring interaction renormalisation of Fermi velocity}

From Eqs. (3.27) \& 4.38), one will find that the linear magneto-tunnelling conductance $G^{(\operatorname{sg})}(0)$ depends on the pseudo-spin structure, the intersecting Fermi circles, and the density of states at the Fermi energy. In this section, we investigate how the presence of electron-electron interactions could affect the magneto-tunnelling conductance.

To introduce electron-electron interactions, one can use many-body theory [53, 70$]$. The Dyson equation for a single particle retarded Green's function can be written as 70 .

$$
\mathcal{G}_{\alpha \beta}^{\mathrm{R}}(\mathbf{k}, \varepsilon)=\mathcal{G}_{\alpha \beta}^{\mathrm{R}, 0}(\mathbf{k}, \varepsilon)+\sum_{\gamma \eta} \mathcal{G}_{\alpha \gamma}^{\mathrm{R}, 0}(\mathbf{k}, \varepsilon) \Sigma_{\gamma \eta}(\mathbf{k}, \varepsilon) \mathcal{G}_{\eta \beta}^{\mathrm{R}}(\mathbf{k}, \varepsilon)
$$

where $\mathcal{G}_{\alpha \beta}^{\mathrm{R}, 0}(\mathbf{k}, \varepsilon)$ is the non-interacting Green's function, and $\Sigma_{\gamma \eta}(\mathbf{k}, \varepsilon)$ is the self energy. The self energy can be written as a series of electron-electron interaction processes within the system. By adding different terms of the series, one can estimate the effect of the full interaction process. In particular, the self energy allows one to renormalise aspects of the band structure due to interactions.

If one writes the Green's functions and self energy as matricies in pseudo-spin, Eq. (5.52) can be rearranged into the form

$$
\mathcal{G}^{\mathrm{R}}(\mathbf{k}, \varepsilon)=\left[\left[\mathcal{G}^{\mathrm{R}, 0}(\mathbf{k}, \varepsilon)\right]^{-1}-\Sigma(\mathbf{k}, \varepsilon)\right]^{-1}
$$

For SLG this can be written as[71]

$$
\mathcal{G}^{\mathrm{R}}(\mathbf{k}, \varepsilon)=\left[\varepsilon \sigma_{0}-v^{0} \hbar \mathbf{k} \cdot \vec{\sigma}-\Sigma(\mathbf{k}, \varepsilon)\right]^{-1}
$$

where $v^{0}$ is the Fermi velocity in SLG with no interactions, $\sigma_{0}$ and $\vec{\sigma}$ are the Pauli matrices. $\mathcal{G}^{\mathrm{R}}(\mathbf{k}, \varepsilon)$ has been written as a matrix in pseudo-spin states. When $\mathcal{G}^{\mathrm{R}}(\mathbf{k}, \varepsilon)$ is diagonal in pseudo-spin, one can calculate the band structure as [70, 72]

$$
\mathcal{A}_{\sigma}(\mathbf{k}, \varepsilon)=\frac{-2 \Im\left\{\Sigma_{\sigma}(\mathbf{k}, \varepsilon)\right\}}{\left[\varepsilon-\sigma v^{0} \hbar k-\Re\left\{\Sigma_{\sigma}(\mathbf{k}, \varepsilon)\right\}\right]^{2}+\left[\Im\left\{\Sigma_{\sigma}(\mathbf{k}, \varepsilon)\right\}\right]^{2}} .
$$

Here we can see that the real part of the self energy shifts the energy of the band structure, and imaginary part of the self energy provides a finite width. 
The first order diagram approximation of the self energy, the Hartree-Fock contribution, for electron-electron interactions in SLG is [71]

$$
\Sigma(\mathbf{k}, \varepsilon) \approx-\frac{\alpha}{4} \ln \left[\frac{k}{\Lambda}\right] v^{0} \hbar \mathbf{k} \cdot \vec{\sigma}-i 0^{+},
$$

where the cut-off momentum is $\Lambda \gg k$, and the interaction strength is $\alpha \ll 1$. Using the first order self energy to calculate the spectral function, one finds that the velocity for SLG is renormalized

$$
v(k)=v^{0}\left(1-\frac{\alpha}{4} \ln \left[\frac{k}{\Lambda}\right]\right) .
$$

This implies that the new Hamiltonians are

$$
\mathcal{H}_{\mathbf{K}}=v(k) \hbar k\left(\begin{array}{cc}
0 & e^{-i \theta_{\mathbf{k}} / 2} \\
e^{i \theta_{\mathbf{k}} / 2} & 0
\end{array}\right), \mathcal{H}_{\mathbf{K}^{\prime}}=v(k) \hbar k\left(\begin{array}{cc}
0 & e^{-i\left(\pi-\theta_{\mathbf{K}}\right) / 2} \\
e^{i\left(\pi-\theta_{\mathbf{k}}\right) / 2} & 0
\end{array}\right)
$$

with energies $\varepsilon_{\gamma, \sigma, \mathbf{k}}=\sigma v(k) \hbar k$. From the new Hamiltonians, it is clear that the pseudo-spin eigenstates remain unchanged. For the first order diagram of the selfenergy, one only has to renormalise the velocity $v$. (This is also true for the random phase approximation [71]). Further more, the renormalised velocity has radial symmetry, i.e. $v(\mathbf{k})=v(k)$, so one expects most aspects of Eq. (4.38) to be preserved. For instance, the pseudo-spin eigenstates are the same. Also, the Fermi surface will be a Fermi circle when $\varepsilon_{\gamma, \sigma, \mathbf{k}}=\sigma v(k) \hbar k$, so the conductance is still described by two interesting Fermi circles. However, the renormalised velocity will change the density of states at the Fermi energy, changing the pre-factor $G_{0}$.

In particular, one can calculate the density of states at the Fermi energy

$$
\begin{aligned}
\rho_{\mathrm{F}} & =\frac{k_{\mathrm{F}}}{2 \pi \hbar}\left[\frac{1}{v\left(k_{\mathrm{F}}\right)+\frac{\mathrm{d} v}{\mathrm{~d} k}\left(k_{\mathrm{F}}\right) k_{\mathrm{F}}}\right] \\
& =\rho_{\mathrm{F}}^{0}\left[\frac{1}{1-\frac{\alpha}{4}\left(\ln \left[\frac{k_{\mathrm{F}}}{\Lambda}\right]+1\right)}\right],
\end{aligned}
$$

where $\rho_{\mathrm{F}}^{0}=\frac{k_{\mathrm{F}}}{2 \pi v^{0} \hbar}$ is the density of states without interactions. One can now look at how the renormalised velocity changes the scaling of the tunnelling conductance through

$$
G_{0}=\frac{g_{\mathrm{s}} g_{\mathrm{v}} \mathrm{e}^{2}}{2 \pi v_{0}^{2} \hbar^{3}} \operatorname{Tr}\left[\tau^{\dagger} \tau\right] A \frac{\rho_{\mathrm{F}}^{(1)} \rho_{\mathrm{F}}^{(2)}}{\rho_{\mathrm{F}}^{0,(1)} \rho_{\mathrm{F}}^{0,(2)}},
$$

which shows that $G_{0} \propto\left[\rho_{\mathrm{F}} / \rho_{\mathrm{F}}^{0}\right]^{2}$ for $k_{\mathrm{F}}^{(1)}=k_{\mathrm{F}}^{(2)}$. One can use $(5.59)$ to see how $G_{0}$ scales for different interactions strengths and different Fermi wave numbers, resulting in the relation

$$
\left[\frac{\rho_{\mathrm{F}}}{\rho_{\mathrm{F}}^{0}}\right]^{2}=\left[\frac{1}{1-\frac{\alpha}{4}\left(\ln \left[\frac{k_{\mathrm{F}}}{\Lambda}\right]+1\right)}\right]^{2} .
$$

Eq. (5.61) can be seen for different values of $\alpha$ in Fig. 5.7, which shows that increasing the interaction strength dampens the tunnelling conductance. 


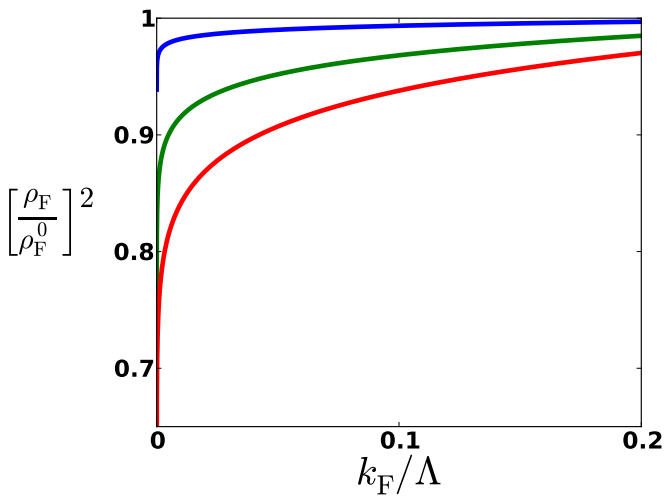

Figure 5.7: $\left[\rho_{\mathrm{F}} / \rho_{\mathrm{F}}^{0}\right]^{2}$ as a function of $k_{\mathrm{F}} / \Lambda$, using Eq. 5.61 for different interaction strengths $\alpha=0.01$ (blue), 0.05 (green), 0.1 (red). Through the relation $G_{0} \propto$ $\left[\rho_{\mathrm{F}} / \rho_{\mathrm{F}}^{0}\right]^{2}$, this plot shows that increasing the interaction strength $\alpha$ dampens the tunnelling conductance. 


\section{Chapter 6}

\section{Non-linear magneto-tunnelling trans- port}

In this chapter, we use linear response theory to obtain an analytic description of the finite bias magneto-tunnelling current and conductance between two layers of SLG in the presence of an in-plane magnetic field (see Fig. 6.1). In partciular, we extend the previous work reviewed in section 3.2 .3 . This chapter also lays out the ground work for finding an analytic finite bias magneto-tunnelling conductance and current between BLG and $\mathrm{MoS}_{2}$ layers, which one could investigate in the future.

To calculate the magneto-tunnelling current and conductance for an in-plane magnetic field, we use Eqs. (3.18) \& (3.19).

We start with the assumption that the momentum-resolved pseudo-spin tunnelling matrix is momentum independent, $\tau_{\mathbf{k}}=\tau$. To avoid a cumbersome formula we choose $\tau=\frac{\tau_{0}}{\sqrt{2}} \sigma_{0}$ and equal densities in each layer. However, one could extend the work in this chapter to a non-pseudo-spin-conserving barrier, and for different densities in each layer. Using these assumptions, we calculate Eq. (3.18) for SLG

$$
\begin{aligned}
\tilde{G}^{(\mathrm{slg})}(\varepsilon, V)= & G_{0} \sqrt{\frac{(2 \varepsilon-e V)^{2}-(\hbar v Q)^{2}}{(\hbar v Q)^{2}-(e V)^{2}}} \\
& \times \Theta(|2 \varepsilon-e V|-\hbar v Q) \Theta(\hbar v Q-|e V|) .
\end{aligned}
$$

Next we discuss how to interpret the finite bias magneto-tunnelling conductance from Eq. (3.19) and Eq. (6.1).

Fig. 6.2 a) shows the dispersion relations of two layers of SLG, which are separated in momentum by $Q$ and in energy by $e V$. The applied bias $V$ opens up an window of energies $\varepsilon_{\mathrm{F}} \leq \varepsilon \leq \varepsilon_{\mathrm{F}}+e V$ for which electrons can participate in tunnelling.

Fig. 6.2 b) shows that tunnelling at an energy $\varepsilon$ can be described by the intersection of an occupied circle with radius $\varepsilon-e V$ and an unoccupied circle with radius $\varepsilon$. The two intersecting circles suggest that the tunnelling at an energy $\varepsilon$ can be understood using a the zero-bias tunnelling conductance, with a density difference of $\mathrm{eV}$. If one labels $2 v \hbar \bar{k}=2 \varepsilon-e V$ and $v \hbar \Delta=e V$, it is straightforward to see that $\tilde{G}(\varepsilon, V)$ has the same form as the linear magneto-tunnelling conductance in Eq. (4.38), where $\mathrm{eV}$ acts like a difference in densities between the layers. The current contribution at an energy $\varepsilon$, due to a small energy window of thickness $\mathrm{d} \varepsilon$, is $\frac{1}{e} \tilde{G}^{(\operatorname{slg})}(\varepsilon, V) \mathrm{d} \varepsilon$.

Eq. (3.19) shows that the tunnelling current $I^{(\mathrm{slg})}(V)$ can be calculated from integrating $G^{(\mathrm{slg})}(\varepsilon, V)$ over the total window of energies $\varepsilon_{\mathrm{F}} \leq \varepsilon \leq \varepsilon_{\mathrm{F}}+e V$. Since 
$\tilde{G}^{(\operatorname{slg})}(\varepsilon, V)$ can be described by the intersection of an occupied circle with radius $\varepsilon-e V$ and an unoccupied circle with radius $\varepsilon, I^{(\operatorname{slg})}(V)$ can be understood using a stack of intersecting circles between $\varepsilon_{\mathrm{F}} \leq \varepsilon \leq \varepsilon_{\mathrm{F}}+e V$.

The integral in Eq. (3.19) can be evaluated to find an analytic solution of $I^{(\mathrm{slg})}(V)$

$$
\begin{aligned}
\frac{I^{(\mathrm{slg})}(V)}{G_{0}} & =\Theta\left(2 \varepsilon_{\mathrm{F}}+e V-\hbar v Q\right) \frac{1}{4 \sqrt{(\hbar v Q)^{2}-(e V)^{2}}}\left(2 \varepsilon_{\mathrm{F}}+e V\right) \sqrt{\left(2 \varepsilon_{\mathrm{F}}+e V\right)^{2}-(\hbar v Q)^{2}} \\
& -\Theta\left(2 \varepsilon_{\mathrm{F}}-e V-\hbar v Q\right) \frac{1}{4 \sqrt{(\hbar v Q)^{2}-(e V)^{2}}}\left(2 \varepsilon_{\mathrm{F}}-e V\right) \sqrt{\left(2 \varepsilon_{\mathrm{F}}-e V\right)^{2}-(\hbar v Q)^{2}} \\
& +\Theta\left(2 \varepsilon_{\mathrm{F}}+e V-\hbar v Q\right) \frac{(\hbar v Q)^{2}}{4 \sqrt{(\hbar v Q)^{2}-(e V)^{2}}} \ln \left[\frac{\hbar v Q}{2 \varepsilon_{\mathrm{F}}+e V+\sqrt{\left(2 \varepsilon_{\mathrm{F}}+e V\right)^{2}-(\hbar v Q)^{2}}}\right] \\
& +\Theta\left(2 \varepsilon_{\mathrm{F}}-e V-\hbar v Q\right) \frac{(\hbar v Q)^{2}}{4 \sqrt{(\hbar v Q)^{2}-(e V)^{2}}} \ln \left[\frac{2 \varepsilon_{\mathrm{F}}-e V+\sqrt{\left(2 \varepsilon_{\mathrm{F}}-e V\right)^{2}-(\hbar v Q)^{2}}}{\hbar v Q}\right]
\end{aligned}
$$

If one differentiates the tunnelling current with respect to $V$, one will find the finite bias magneto-tunnelling conductance

$$
\begin{aligned}
\frac{G^{(\mathrm{slg})}(V)}{G_{0}} & =\Theta\left(2 \varepsilon_{\mathrm{F}}+e V-\hbar v Q\right) \frac{1}{2} \sqrt{\frac{\left(2 \varepsilon_{\mathrm{F}}-e V\right)^{2}-(\hbar v Q)^{2}}{(\hbar v Q)^{2}-(e V)^{2}}} \\
& +\Theta\left(2 \varepsilon_{\mathrm{F}}-e V-\hbar v Q\right) \frac{1}{2} \sqrt{\frac{\left(2 \varepsilon_{\mathrm{F}}+e V\right)^{2}-(\hbar v Q)^{2}}{(\hbar v Q)^{2}-(e V)^{2}}} \\
& +\Theta\left(2 \varepsilon_{\mathrm{F}}+e V-\hbar v Q\right) \frac{V}{4\left[(\hbar v Q)^{2}-(e V)^{2}\right]^{\frac{3}{2}}}\left[\left(2 \varepsilon_{\mathrm{F}}+e V\right) \sqrt{\left(2 \varepsilon_{\mathrm{F}}+e V\right)^{2}-(\hbar v Q)^{2}}\right] \\
& +\Theta\left(2 \varepsilon_{\mathrm{F}}-e V-\hbar v Q\right) \frac{V}{4\left[(\hbar v Q)^{2}-(e V)^{2}\right]^{\frac{3}{2}}}\left[\left(2 \varepsilon_{\mathrm{F}}-e V\right) \sqrt{\left(2 \varepsilon_{\mathrm{F}}-e V\right)^{2}-(\hbar v Q)^{2}}\right] \\
& +\Theta\left(2 \varepsilon_{\mathrm{F}}+e V-\hbar v Q\right) \frac{(\hbar v Q)^{2} V}{4\left[(\hbar v Q)^{2}-(e V)^{2}\right]^{\frac{3}{2}}} \ln \left[\frac{\hbar v Q}{2 \varepsilon_{\mathrm{F}}+e V+\sqrt{\left(2 \varepsilon_{\mathrm{F}}+e V\right)^{2}-(\hbar v Q)^{2}}}\right] \\
& +\Theta\left(2 \varepsilon_{\mathrm{F}}-e V-\hbar v Q\right) \frac{(\hbar v Q)^{2} V}{4\left[(\hbar v Q)^{2}-(e V)^{2}\right]^{\frac{3}{2}}} \ln \left[\frac{2 \varepsilon_{\mathrm{F}}-e V+\sqrt{\left(2 \varepsilon_{\mathrm{F}}-e V\right)^{2}-(\hbar v Q)^{2}}}{\hbar v Q}\right]
\end{aligned}
$$

which can be seen as a function of $Q$ and $V$ in Fig. 6.3. From this figure and Fig. 6.4, one can see different characteristics in the regions $0<v \hbar Q<e V, e V<v \hbar Q<$ $2 \varepsilon_{\mathrm{F}}-e V$, and $2 \varepsilon_{\mathrm{F}}-e V<v \hbar Q<2 \varepsilon_{\mathrm{F}}+e V$. When $v \hbar Q<e V$, the two Dirac cones do not intersect, so no tunnelling is allowed. At $v \hbar Q=e V$, the two conical surfaces start to intersect with maximum pseudo-spin overlap, this gives rise to a resonance in the tunnelling conductance. For $e V<v \hbar Q<2 \varepsilon_{\mathrm{F}}-e V$, the characteristics originate from the stacked pairs of intersecting circles, and the pseudo-spin overlap at each of their intersections. For $v \hbar Q$ near $\mathrm{eV}$, the finite bias tunnelling conductance is greater than the zero bias tunnelling conductance. This is because there are more 


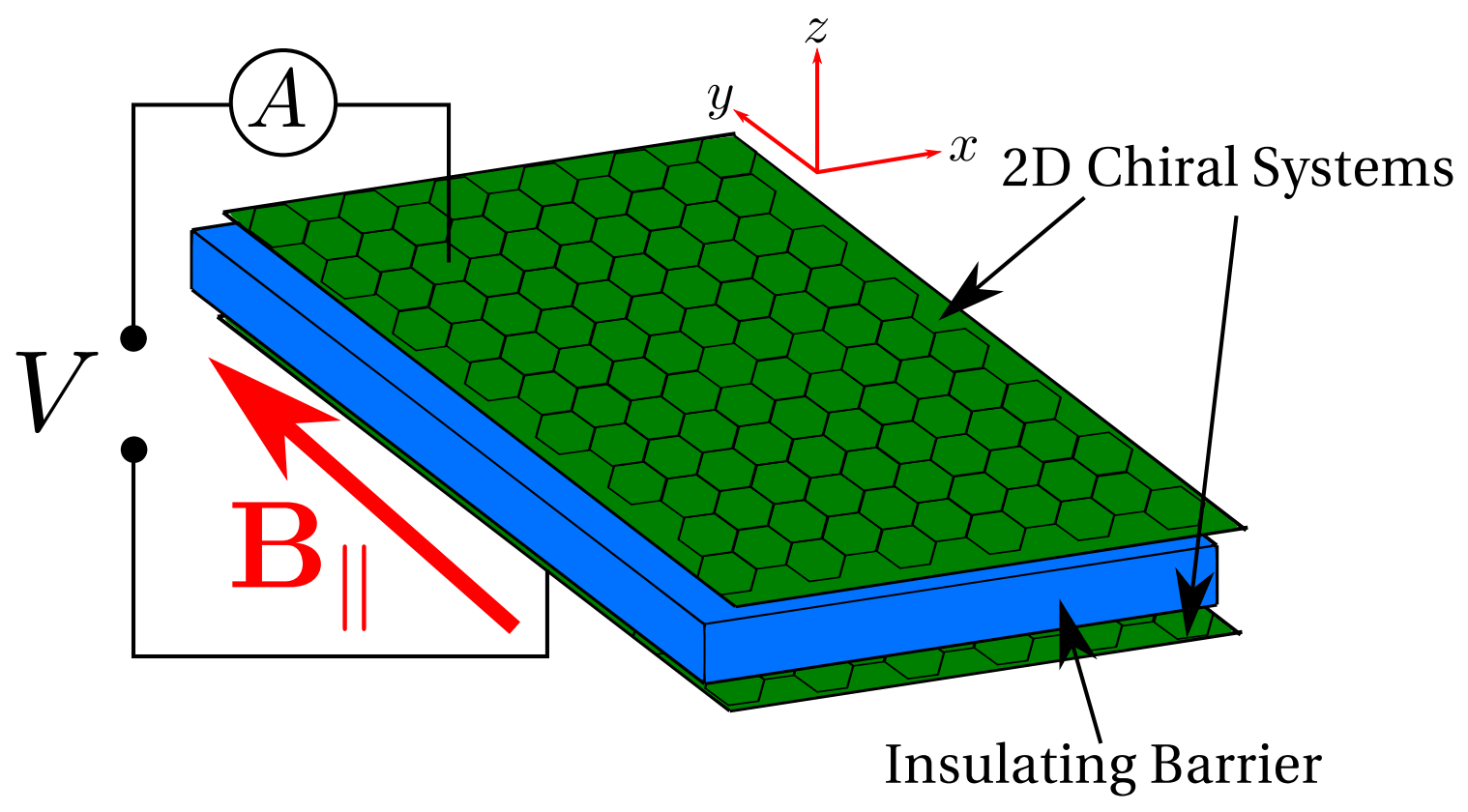

Figure 6.1: Two parallel layers of SLG (green) are separated by an insulating barrier (blue). There is a finite bias between the layers, and an in-plane magnetic field is applied. In this chapter we derive the finite bias magneto-tunnelling current and conductance for this system.

electrons tunnelling within the energy window. However, the finite bias tunnelling conductance dips below the zero-bias tunnelling conductance as $v \hbar Q$ increases. When $v \hbar Q=2 \varepsilon_{\mathrm{F}}-e V$, the intersecting circles at $\varepsilon=\varepsilon_{\mathrm{F}}$ cease to intersect, this generates a kink in the tunnelling conductance. The intersecting circles completely separate over the region $2 \varepsilon_{\mathrm{F}}-e V<v \hbar Q<2 \varepsilon_{\mathrm{F}}+e V$. At each separation, the pseudo-spins are orthogonal, which explains why there is no divergence over this region.

In this chapter, we have linked the linear magneto-tunnelling conductance to the non-linear magneto-tunnelling conductance, for tunnelling between two layers of SLG. Using Eq. 3.19), one can explore other examples of chiral and non-chiral magneto-tunnelling transport with a finite bias by relating each example to the zerobias case. 

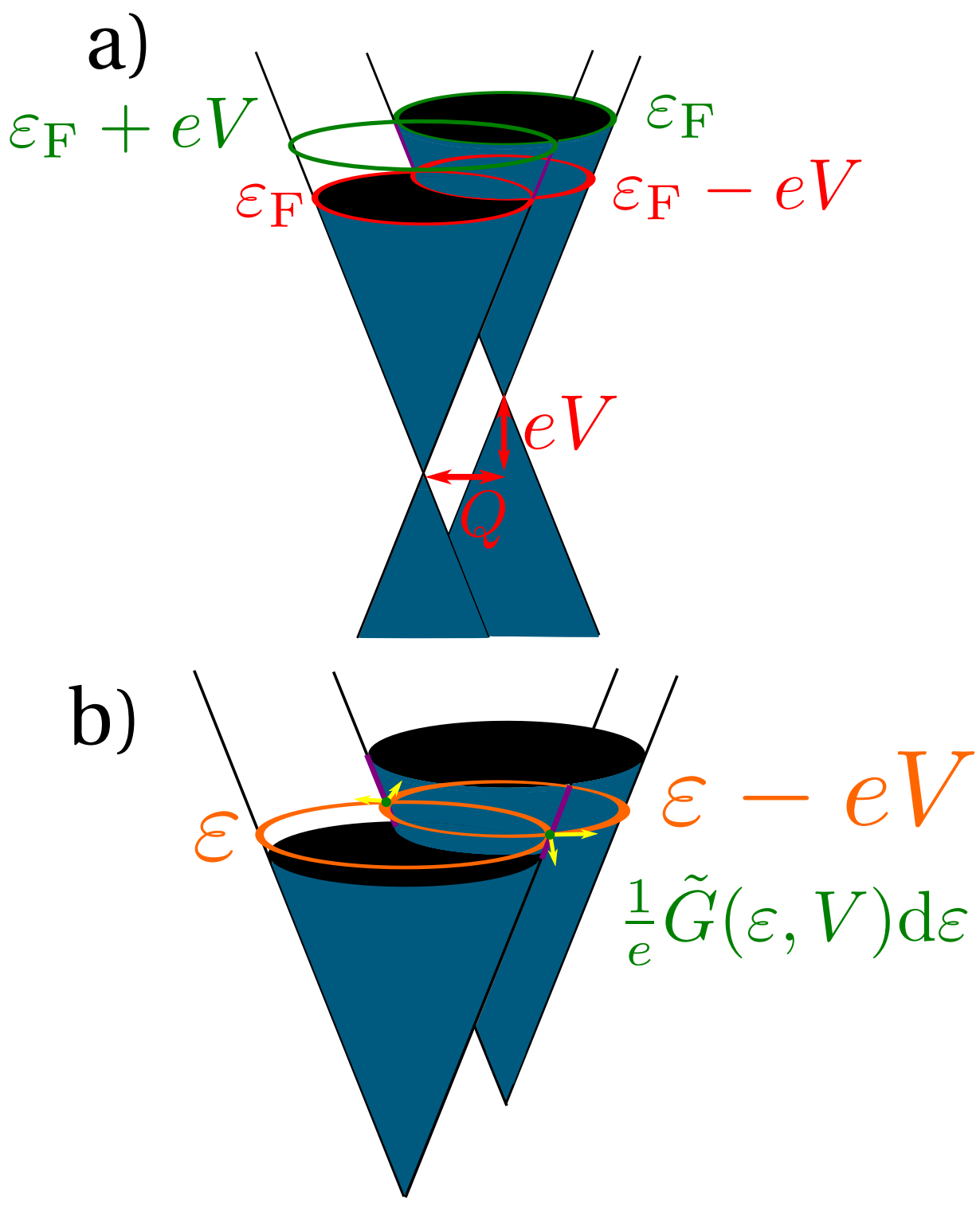

Figure 6.2: a) Two intersecting Dirac cones that have been shifted by a magnetic field and applied bias, as shown by $Q$ and $e V$. Tunnelling can only occur for states where the conical surfaces intersect (purple). The energy window at which tunnelling occurs can be seen in red $\left(\varepsilon_{\mathrm{F}}\right)$ and green $\left(\varepsilon_{\mathrm{F}}+e V\right)$. b) The tunnelling that occurs can be described using two intersecting circles (orange), an unoccupied circle of radius $\varepsilon$ and an occupied circle of radius $\varepsilon-e V$. At an energy $\varepsilon$, the current contribution where the circles intersect is $\frac{1}{e} \tilde{G}(\varepsilon, V) \mathrm{d} \varepsilon$ (green dots). The pseudo-spin vectors for each intersection can be seen in yellow. The finite bias tunnelling current characteristics can be understood from using a stack of intersecting circles (in orange) from $\varepsilon_{\mathrm{F}}$ to $\varepsilon_{\mathrm{F}}+e V$. 

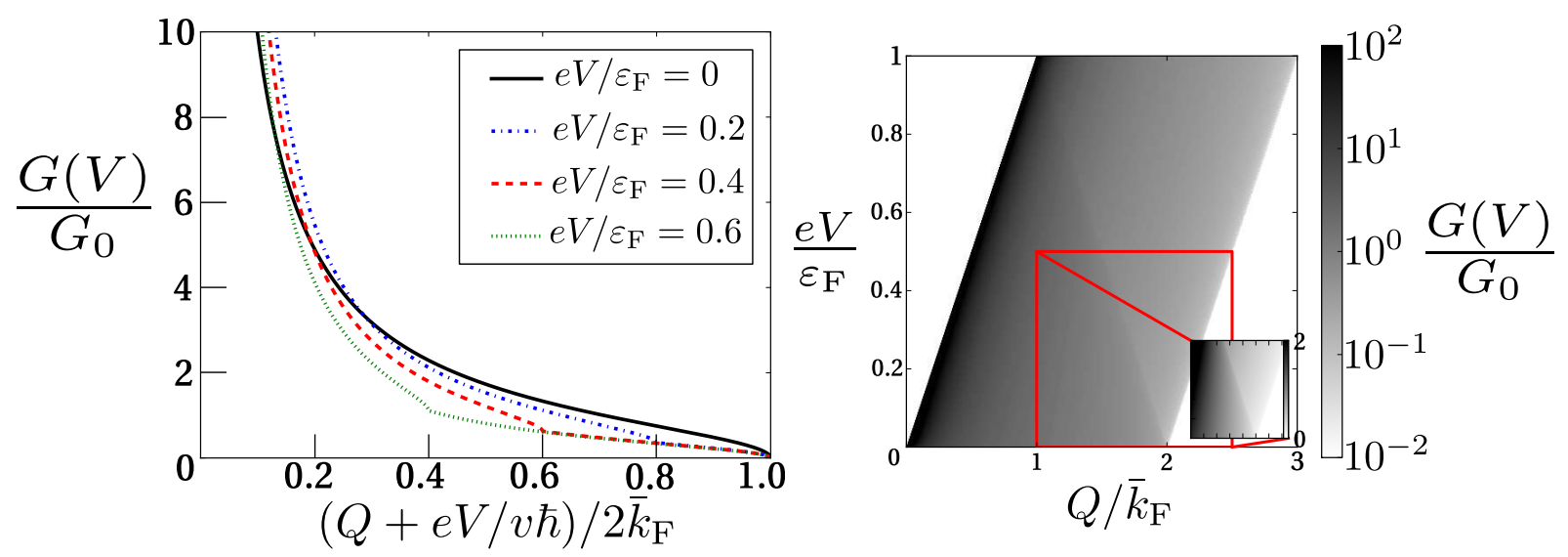

Figure 6.3: (Left)The finite bias magneto-tunnelling conductance ploted as a function of $\frac{Q+e V / v \hbar}{2 \bar{k}_{\mathrm{F}}}$, for $\mathrm{eV} / \varepsilon_{\mathrm{F}}=0$ (black) $\mathrm{eV} / \varepsilon_{\mathrm{F}}=0.2$ (green) $\mathrm{eV} / \varepsilon_{\mathrm{F}}=0.4$ (red), $\mathrm{eV} / \varepsilon_{\mathrm{F}}=0.6$ (blue). We see that the finite bias conductance dips below the zero-bias conductance, this is because of the pseudo-spin overlap at the intersection of the two cones. We can also see a kink at $v \hbar Q=2 \varepsilon_{\mathrm{F}}-e V$, where there is a change in behaviour due to the current switching off. (Right) The magneto-tunnelling conductance plotted as a function of $Q$ and $V$. One can see the divergence where the cones intersect, and the termination when the cones are completely separated. In the small window with the adjusted scale, one can see the kink where the current starts to switch off (see Fig. 6.4).
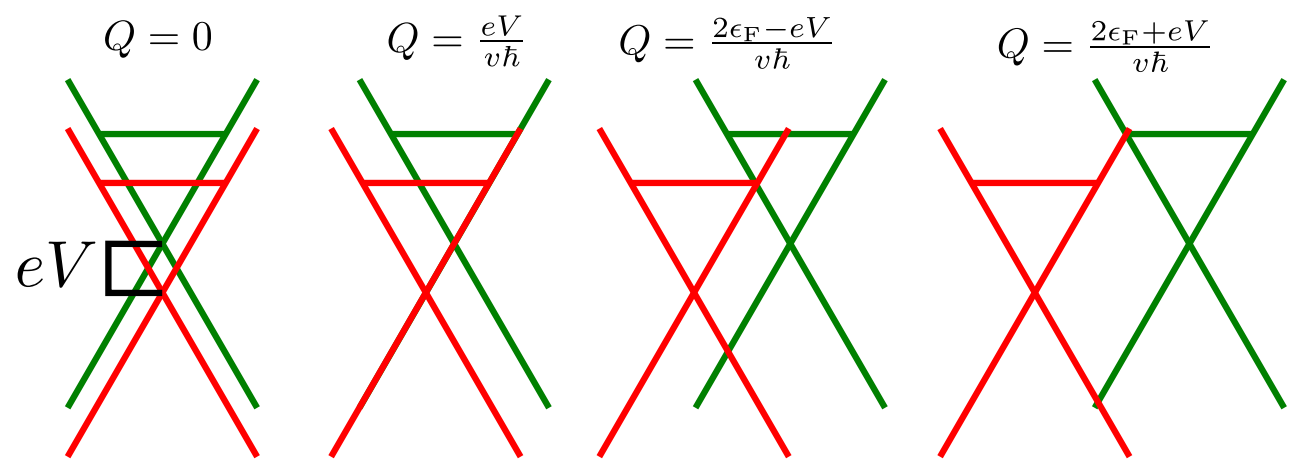

Figure 6.4: A series of Dirac cones for equal densities, separated by an applied bias $e V$ and momentum $Q$. The horizontal lines represent the Fermi level, all states below the horizontal lines are occupied. When $v \hbar Q=0$, the Dirac cones do not intersect so there is no current. However, once $v \hbar Q=e V$ the cones intersect, and the pseudo-spins have a full overlap at this boundary, which produces a divergence in the tunnelling conductance. For the region $e V<v \hbar Q<2 \varepsilon_{\mathrm{F}}+e V$, only states between the Fermi levels $\left(\varepsilon_{\mathrm{F}}<\varepsilon<\varepsilon_{\mathrm{F}}+e V\right)$ of each cone can tunnel. At $v \hbar Q=2 \varepsilon_{\mathrm{F}}-e V$, the current starts to turn off because the cones start to separate, this causes a kink in the conductance. At each separation point the pseudo-spin overlap is zero. For $v \hbar Q>2 \varepsilon_{\mathrm{F}}-e V$, only states between $v \hbar Q<\varepsilon<2 \varepsilon_{\mathrm{F}}+e V$ can tunnel. When $v \hbar Q>2 \varepsilon_{\mathrm{F}}+e V$, particles do not occupy the states that would conserve energy and momentum in the tunnelling process, so the conductance is zero. 


\section{Chapter 7}

\section{Conclusion}

We theoretically studied magneto-tunnelling transport between two parallel twodimensional chiral electron gases. The motivations for doing so stem from past work on magneto-tunnelling transport between two parallel two-dimensional nonchiral electron gases, and the recent fabrication of double layer van der Waals heterostructures which have a chiral structure. This thesis extends previous knowledge in magneto-tunnelling transport to chiral charge carriers, in a way that is relevant to current research. We show how magneto-tunnelling be used to probe the lattice structure of a van der Waals heterostructure, and how magneto-tunnelling can be used to generate a valley polarized current in a van der Waals heterostructure. Significant results from this thesis can be found in Refs. [1, 2].

At the start of this work, we preformed a review of magneto-tunnelling transport. We discussed energy-momentum conservation in the tunnelling process, and we also introduced our assumptions of zero temperature, no interactions or disorder. This review allowed us to extend the previous work of magneto-tunnelling transport between non-chiral electron gases, to the new work of magneto-tunnelling transport between chiral electron gases. We will now conclude this MSc thesis by summarising the new and relevant understanding obtained from this work.

To investigate the tunnelling of chiral charge carriers, we extended the tunnelling matrix to include pseudo-spin tunnelling processes. We represented the pseudospin tunnelling matrix using the basis of Pauli matrices, where each Pauli matrix represents a possible pseudo-spin flip in the tunnelling process. This basis for the tunnelling matrix provides clarity in the results, as it relates terms in the magnetotunnelling conductance to the types of tunnelling processes.

In general, the momentum dependent pseudo-spin states that conserve energy and momentum in a tunnelling event are responsible for the change in magnetotunnelling characteristics from that of a non-chiral electron gas. This could be seen in the linear magneto-tunnelling conductance characteristics for tunnelling between two single layer graphene, bilayer graphene, and $\mathrm{MoS}_{2}$ layers in an in-plane magnetic field. The pseudo-spin states at the intersections of the Fermi circles change as a function of the Fermi circle separation, this changes the pseudo-spin overlap between the final and initial tunnelling states as a function of the in-plane magnetic field. Further more, the pseudo-spin tunnelling matrix may alter the pseudo-spin states in the tunnelling process. Both the momentum dependence of pseudo-spin and the altering of pseudo-spin states in the tunnelling process explain the resonances and features of the linear magneto-tunnelling conductance between two parallel two dimensional 
chiral electron gases.

When pseudo-spin is conserved, it was shown that the pseudo-spin overlap between tunnelling states would dampen the linear magneto-tunnelling conductance characteristics from that of the non-chiral tunnelling. This explains the tunnelling characteristics for single layer graphene, bilayer graphene, and $\mathrm{MoS}_{2}$ double layer systems. In $\mathrm{MoS}_{2}$, the chirality of the pseudo-spin eigenstates depends on how close the Fermi energy is to the band gap. By shifting the Fermi energy in each layer of $\mathrm{MoS}_{2}$, one can tune the characteristics of the linear magneto-tunnelling conductance.

For a tilted magnetic field in single layer graphene, the magneto-tunnelling conductance was also found to depend on pseudo-spin overlap between energy-momentum conserving states. In this case, pseudo-spin overlap consists of overlapping Landau levels (a pair of cyclotron orbits). The orbits are determined by the out of plane component of the magnetic field, and the in-plane component of the magnetic field shifts guiding centres for these orbits between the layers. As one shifts the guiding centres of the orbits between the layers using an in-plane magnetic field, one will see the wave function overlap between the layers oscillate. These oscillations can then be seen in the magneto-tunnelling conductance, since it is proportional to the wave function overlap. Again, the pseudo-spin tunnelling matrix allows for different tunnelling processes, allowing for the different oscillations in wave-function overlap. For zero in-plane magnetic field, the relevant pseudo-spin structure of the tunnelling matrix simplifies, and becomes sensitive to the Fermi energies in each layer.

In this work, we discussed how linear magneto-tunnelling transport can be employed as a tool. We showed how it can be used to investigate the properties of a double layer van der Waals heterostructure, or to generate a valley polarised current between single and bilayer graphene.

We explicitly demonstrated how measurements of the linear magneto-tunnelling conductance between two layers of single layer graphene can be used to measure properties of the tunnelling matrix. We suggest using a least squares approach to fit the curve to a polynomial, to obtain parameters that interdependent on the pseudospin tunnel-coupling. Theory predicts that the fitted parameters will be dependent, this allows one to test if theory is consistent with experiment. The pseudo-spin tunnel-coupling is expected to depend on the atomic lattice structure between the layers. Measuring the pseudo-spin tunnel-coupling allows one to probe this structure, providing a new spectroscopic tool.

For two parallel tunnel-coupled single layer and bilayer graphene systems, we calculated the linear magneto-tunnelling conductance for an in-plane magnetic field. In particular, we found conditions in which the tunnel-coupling would allow a valley polarised current. When pseudo-spin is conserved in the tunnelling process, we calculate the valley polarisation of the current as a function of the maximum magnetic field. This method for generating a valley polarised current is dependent on the applied magnetic field and the pseudo-spin tunnel-coupling between the single layer and bilayer graphene systems. It is distinct from methods suggsted in other works which require nanostructuring of a graphene sample [44, 57], coupling to electromagnetic fields [58, 66], application of mechanical strain [61 63, 66 68], or the presence 
of defects 69. This new method for generating a valley polarised current may be important for creating valleytronic information processing devices.

The effect of electron-electron interactions in single layer graphene on magnetotunnelling transport was investigated. For two parallel tunnel-coupled layers of graphene with an applied in-plane magnetic field, the first order self energy diagram was used to calculate the effect on the linear magneto-tunnelling conductance. From this calculation, it was found that increasing the interaction strength will dampen the magnitude of the tunnelling conductance, while not effecting the characteristics.

The effect of an in-plane electric field on magneto-tunnelling transport was investigated. The linear magneto-tunnelling conductance between two single layer graphene layers was calculated with an in-plane magnetic field, when an in-plane electric field was perpendicular or parallel to the magnetic field. The in-plane electric field changes the Fermi surface from a Fermi circle to a Fermi ellipse. Therefore, the linear magneto-tunnelling conductance can be explained by two intersecting Fermi ellipses, separated due to a magnetic field. The electric field determines the shape of each ellipse, which determines how the Fermi circles start/cease to intersect, therefore the electric field will change the resonance locations in the linear magneto-tunnelling conductance.

The non-linear magneto-tunnelling transport between two parallel single graphene layers was studied. We calculated the magneto-tunnelling current and conductance in the presence of an in-plane magnetic field. We explained how the magnetotunnelling current is calculated from integrating over the intersection of the two energy-momentum relations, separated in momentum by the in-plane magnetic field and in energy by the applied bias between the layers. We discussed how the magnetotunnelling current could be related to the understanding of the linear magnetotunnelling conductance. We described how features in the conductance relate to various intersecting energy-momentum relations and the pseudo-spin overlap between the states at the intersection.

Looking towards the future, there are multiple research avenues to explore. To point this out, we list two avenues that we are aware of, and suggest why they could be worth investigating.

For experimental research, one could use measurements of the magneto-tunnelling conductance between tunnel-coupled two-dimensional chiral electron gases, and compare the measurements with the theory in this work. In the case that experiment is consistent with theory, it should be possible to test the new spectroscopic methods suggested in this work. This may lead to an experimental understanding of pseudo-spin tunnel-coupling.

To move forward in theoretical aspects of magneto-tunnelling, one can investigate magneto-tunnelling spin transport between two-dimensional chiral electron gases. In this topic, one could start with the spin polarised non-linear magneto-tunnelling transport between the electronic surface states of a thin topological insulator film (which has momentum coupled with real spin eigenstates). In Ref. [25], the linear magneto-tunnelling conductance was calculated for a charge and spin tunnelling current between the two surfaces of the film. However, extending chapter 6 of this 
work, one can derive an analytic formula for the finite bias magneto-tunnelling conductance for the spin and charge current across a thin topological insulator film. Only recently, Ref. [73] experimentally investigated the band-structure of $\mathrm{Bi}_{2} \mathrm{Se}_{3}$ thin films in the quantum tunnelling limit. A theoretical understanding of magnetotunnelling spin transport between surface states of a thin film topological insulator could aid experimental efforts. 


\section{Bibliography}

[1] L. Pratley and U. Zülicke, Magnetotunneling spectroscopy of chiral twodimensional electron systems, Phys. Rev. B 88, 245412 (2013).

[2] L. Pratley and U. Zülicke, Valley filter from magneto-tunneling between single and bi-layer graphene, Applied Physics Letters 104, 082401 (2014).

[3] K. S. Novoselov et al., Electric Field Effect in Atomically Thin Carbon Films, Science 306, 666 (2004).

[4] K. S. Novoselov et al., A roadmap for graphene, Nature 490, 192 (2012).

[5] A. K. Geim, Graphene: status and prospects, Science 324, 1530 (2009).

[6] A. K. Geim and A. H. MacDonald, Graphene: Exploring Carbon Flatland, Physics Today 60, 080000 (2007).

[7] R. Winkler and U. Zülicke, Invariant expansion for the trigonal band structure of graphene, Phys. Rev. B 82, 245313 (2010).

[8] A. C. Neto et al., The electronic properties of graphene, Reviews of modern physics 81, 109 (2009).

[9] K. Novoselov et al., Two-dimensional atomic crystals, Proceedings of the National Academy of Sciences of the United States of America 102, 10451 (2005).

[10] M. Xu, T. Liang, M. Shi, and H. Chen, Graphene-Like Two-Dimensional Materials, Chemical Reviews 113, 3766 (2013).

[11] S. Z. Butler et al., Progress, Challenges, and Opportunities in Two-Dimensional Materials Beyond Graphene, ACS Nano 7, 2898 (2013).

[12] A. K. Geim and I. V. Grigorieva, Van der Waals heterostructures, Nature (London) 499, 419 (2013).

[13] W. Greiner, Relativistic Quantum Mechanics. Wave Equations (U.S. Government Printing Office, ADDRESS, 2000).

[14] R. Winkler, Spin-orbit Coupling Effects in Two-Dimensional Electron and Hole Systems, No. no. 191 in Physics and Astronomy Online Library (Springer, ADDRESS, 2003).

[15] L. Britnell et al., Field-Effect Tunneling Transistor Based on Vertical Graphene Heterostructures, Science 335, 947 (2012). 
[16] T. Georgiou et al., Vertical field-effect transistor based on graphene- $W S_{2}$ heterostructures for flexible and transparent electronics, Nat. Nanotech. 8, 100 (2012).

[17] L. Britnell et al., Electron Tunneling through Ultrathin Boron Nitride Crystalline Barriers, Nano Lett. 12, 1707 (2012).

[18] L. Britnell et al., Resonant tunnelling and negative differential conductance in graphene transistors, Nat. Comms. 4, 1794 (2013).

[19] C. R. Dean et al., Boron nitride substrates for high-quality graphene electronics, Nat. Nanotech. 5, 722 (2010).

[20] R. V. Gorbachev et al., Strong Coulomb drag and broken symmetry in doublelayer graphene, Nature Physics (2012).

[21] F. T. Vasko, Resonant and nondissipative tunneling in independently contacted graphene structures, Phys. Rev. B 87, 075424 (2013).

[22] O. E. Raichev and F. T. Vasko, Non-dissipative tunnelling between lowdimensional electron states in a magnetic field, J. Phys.: Condens. Matter 8, 1041 (1996).

[23] L. Zheng and A. H. MacDonald, Tunneling conductance between parallel twodimensional electron systems, Phys. Rev. B 47, 10619 (1993).

[24] S. K. Lyo, Quantum oscillations of two-dimensional to two-dimensional tunneling in bilayer electron gases in tilted magnetic fields, Phys. Rev. B 57, 9114 (1998).

[25] S. S. Pershoguba and V. M. Yakovenko, Spin-polarized tunneling current through a thin film of a topological insulator in a parallel magnetic field, Phys. Rev. B 86, 165404 (2012).

[26] E. L. Wolf, Principles of Electron Tunneling Spectroscopy (Oxford University Press, New York, 1985).

[27] C. Chen, Introduction to scanning tunneling microscopy, Monographs on the physics and chemistry of materials (Oxford University Press, New York, 2008).

[28] J. Smoliner, E. Gornik, and G. Weimann, Direct observation of tunneling between Landau levels in barrier-separated two-dimensional electron-gas systems, Phys. Rev. B 39, 12937 (1989).

[29] J. Smoliner et al., Momentum conservation in tunneling processes between barrier-separated 2D-electron-gas systems, Phys. Rev. Lett. 63, 2116 (1989).

[30] J. P. Eisenstein, T. J. Gramila, L. N. Pfeiffer, and K. W. West, Probing a two-dimensional Fermi surface by tunneling, Phys. Rev. B 44, 6511 (1991). 
[31] U. Gennser et al., Probing band structure anisotropy in quantum wells via magnetotunneling, Phys. Rev. Lett. 67, 3828 (1991).

[32] J. A. Simmons et al., Submicrometer control of two-dimensional-twodimensional magnetotunneling in double-well heterostructures, Phys. Rev. B 47, 15741 (1993).

[33] N. K. Patel et al., Lateral transport studies of coupled electron gases, Semicond. Sci. Technol. 11, 703 (1996).

[34] J. E. Hasbun, Conductance in double quantum well systems, J. Phys.: Condens. Matter 15, R143 (2003).

[35] C. C. Eugster, J. A. del Alamo, M. J. Rooks, and M. R. Melloch, Onedimensional to one-dimensional tunnelling between electron waveguides, Appl. Phys. Lett. 64, 3157 (1994).

[36] J. Wang et al., Resonant Magnetotunneling via One-Dimensional Quantum Confined States, Phys. Rev. Lett. 73, 1146 (1994).

[37] O. M. Auslaender et al., Tunneling Spectroscopy of the Elementary Excitations in a One-Dimensional Wire, Science 295, 825 (2002).

[38] E. Bielejec, J. A. Seamons, J. L. Reno, and M. P. Lilly, Tunneling and nonlinear transport in a vertically coupled GaAs/AlGaAs double quantum wire system, Appl. Phys. Lett. 86, (2005).

[39] E. E. Vdovin et al., Imaging the electron wave function in self-assembled quantum dots, Science 290, 122 (2000).

[40] G. Rainer et al., Tunneling and nonparabolicity effects in in-plane magnetic fields, Phys. Rev. B 51, 17642 (1995).

[41] S. Lyo et al., Nonlinear resonant tunneling in low-dimensional systems in a magnetic field: Energy dispersion, Physica E 34, 425 (2006).

[42] E. Bielejec et al., Tunneling and nonlinear transport in a low-dimensional vertically coupled GaAs/AlGaAs system, Physica E 34, 433 (2006).

[43] O. Gunawan, B. Habib, E. P. De Poortere, and M. Shayegan, Quantized conductance in an AlAs two-dimensional electron system quantum point contact, Phys. Rev. B 74, 155436 (2006).

[44] J. T. A Rycerz et al., Valley filter and valley valve in graphene, Nature Physics 3, 172 (2007).

[45] D. Xiao, W. Yao, and Q. Niu, Valley-Contrasting Physics in Graphene: Magnetic Moment and Topological Transport, Phys. Rev. Lett. 99, 236809 (2007). 
[46] A. H. Castro Neto et al., The electronic properties of graphene, Rev. Mod. Phys. 81, 109 (2009).

[47] E. McCann and M. Koshino, The electronic properties of bilayer graphene, Reports on Progress in Physics 76, 056503 (2013).

[48] E. McCann and V. I. Fal'ko, Landau-Level Degeneracy and Quantum Hall Effect in a Graphite Bilayer, Phys. Rev. Lett. 96, 086805 (2006).

[49] B. Mills, Molybdenite-3D-balls.png, http://commons.wikimedia.org/wiki/ File:Molybdenite-3D-balls.png, 2007, [Online; accessed 22-January-2014].

[50] A. Kormányos et al., Monolayer $\mathrm{MoS}_{2}$ : Trigonal warping, the $\Gamma$ valley, and spin-orbit coupling effects, Phys. Rev. B 88, 045416 (2013).

[51] D. Xiao et al., Coupled Spin and Valley Physics in Monolayers of $\mathrm{MoS}_{2}$ and Other Group-VI Dichalcogenides, Phys. Rev. Lett. 108, 196802 (2012).

[52] C. Kittel and H. Kroemer, Thermal Physics (W. H. Freeman, New York, 1980).

[53] H. Bruus and K. Flensberg, Many-Body Quantum Theory in Condensed Matter Physics: An Introduction (Oxford University Press, Oxford, UK, 2004).

[54] A. H. MacDonald, Mesoscopic Quantum Physics (Elsevier, Amsterdam, 1995), pp. 659,720, edited by E. Akkermans et al.

[55] J. Hu and A. H. MacDonald, Electronic structure of parallel two-dimensional electron systems in tilted magnetic fields, Phys. Rev. B 46, 12554 (1992).

[56] J. J. J. Sakurai and J. Napolitano, Modern Quantum Mechanics (AddisonWesley, Boston, 2010).

[57] H. Schomerus, Helical scattering and valleytronics in bilayer graphene, Phys. Rev. B 82, 165409 (2010).

[58] W. Yao, D. Xiao, and Q. Niu, Valley-dependent optoelectronics from inversion symmetry breaking, Phys. Rev. B 77, 235406 (2008).

[59] J. L. Garcia-Pomar, A. Cortijo, and M. Nieto-Vesperinas, Fully Valley-Polarized Electron Beams in Graphene, Phys. Rev. Lett. 100, 236801 (2008).

[60] D. S. L. Abergel and T. Chakraborty, Generation of valley polarized current in bilayer graphene, Appl. Phys. Lett. 95, (2009).

[61] T. Fujita, M. B. A. Jalil, and S. G. Tan, Valley filter in strain engineered graphene, Appl. Phys. Lett. 97, (2010).

[62] T. Low and F. Guinea, Strain-Induced Pseudomagnetic Field for Novel Graphene Electronics, Nano Lett. 10, 3551 (2010). 
[63] F. Zhai, X. Zhao, K. Chang, and H. Q. Xu, Magnetic barrier on strained graphene: A possible valley filter, Phys. Rev. B 82, 115442 (2010).

[64] L. E. Golub, S. A. Tarasenko, M. V. Entin, and L. I. Magarill, Valley separation in graphene by polarized light, Phys. Rev. B 84, 195408 (2011).

[65] G. Y. Wu, N.-Y. Lue, and Y.-C. Chen, Quantum manipulation of valleys in bilayer graphene, Phys. Rev. B 88, 125422 (2013).

[66] Z. Khatibi, H. Rostami, and R. Asgari, Valley polarized transport in a strained graphene based Corbino disc, Phys. Rev. B 88, 195426 (2013).

[67] Z. Wu et al., Valley-Dependent Brewster Angles and Goos-Hänchen Effect in Strained Graphene, Phys. Rev. Lett. 106, 176802 (2011).

[68] Y. Jiang et al., Generation of Pure Bulk Valley Current in Graphene, Phys. Rev. Lett. 110, 046601 (2013).

[69] D. Gunlycke and C. T. White, Graphene Valley Filter Using a Line Defect, Phys. Rev. Lett. 106, 136806 (2011).

[70] G. Mahan, Many-Particle Physics, Physics of Solids and Liquids (Springer, New York, 2000).

[71] V. N. Kotov et al., Electron-Electron Interactions in Graphene: Current Status and Perspectives, Rev. Mod. Phys. 84, 1067 (2012).

[72] T. Jungwirth and A. H. MacDonald, Electron-electron interactions and twodimensional - two-dimensional tunneling, Phys. Rev. B 53, 7403 (1996).

[73] G. Landolt et al., Spin Texture of $\mathrm{Bi}_{2} \mathrm{Se}_{3}$ Thin Films in the Quantum Tunneling Limit, Phys. Rev. Lett. 112, 057601 (2014). 